\author{
Aus dem Fachbereich Medizin \\ der Johann Wolfgang Goethe-Universität \\ Frankfurt am Main
}

\author{
betreut am \\ Zentrum der Chirurgie \\ Klinik für Gefäß- und Endovascularchirurgie \\ Direktor: Prof. Dr. Thomas Schmitz-Rixen
}

\title{
Kann Google Glass $₫$ den Arbeitsablauf peripherer endovaskulärer Interventionen verbessern? Eine Pilotstudie mit Google Glass $₫$ im Rahmen einer Virtual Reality Simulation.
}

\author{
Dissertation \\ zur Erlangung des Doktorgrades der Medizin \\ des Fachbereichs Medizin \\ der Johann Wolfgang Goethe-Universität \\ Frankfurt am Main \\ vorgelegt von \\ Dennis Kaczmarek \\ aus Langen
}

Frankfurt am Main, 2020 
Dekan:

Prof. Dr. Stefan Zeuzem

Referent:

Prof. Dr. Thomas Schmitz-Rixen

Korreferent:

Prof. Dr. Thomas Vogl

Tag der mündlichen Prüfung: $\quad$ 10. Mai 2021 
„Die Bäume mit tiefen Wurzeln sind die, die hoch wachsen.“ - Frédéric Mistral (1830 - 1914) -

Für meine Wurzeln, meine Familie 


\section{INHALTSVERZEICHNIS}

WIDMUNG................................................................................................. III

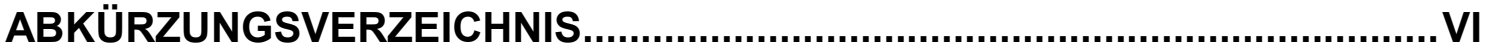

ABBILDUNGS- UND TABELLENVERZEICHNIS ...................................... VII

GENDER-HINWEIS................................................................................ VIII

ZUSAMMENFASSUNG ............................................................................ IX

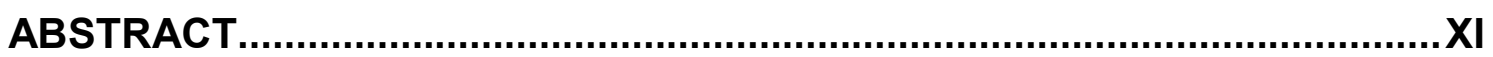

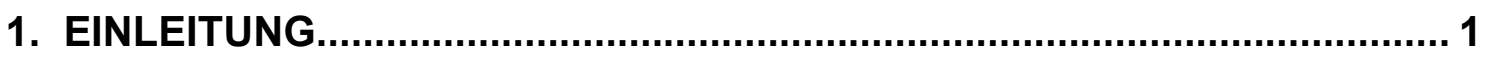

2. PROBANDEN, MATERIAL UND METHODEN ......................................... 3

2.1 Studiendesign.....................................................................

2.2 Probandenkollektiv................................................................ 4

2.3 Google Glass ${ }^{\circledR}$ Explorer Edition...........................................6

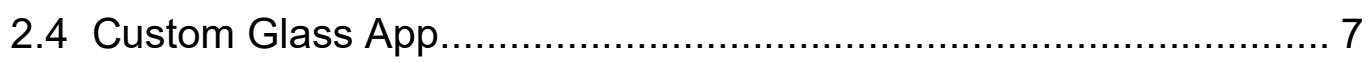

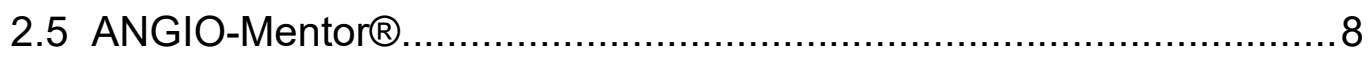

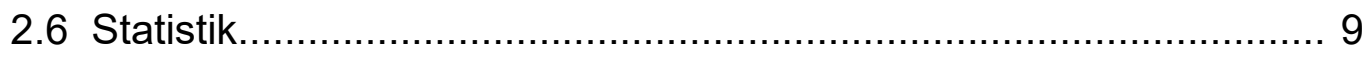

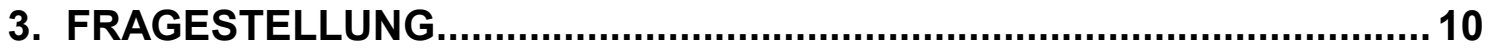

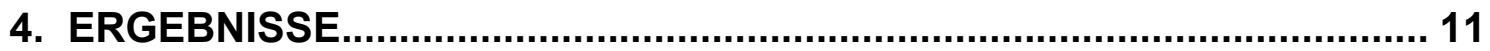

4.1 Auswertung der Bewertungsprotokolle zur Simulation...................11

4.1.1 Gesamtpunktzahl und Simulationszeit........................ 11

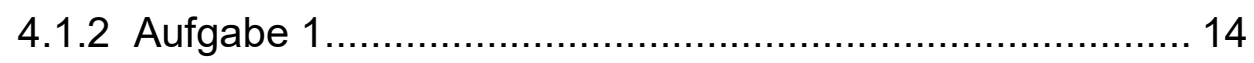

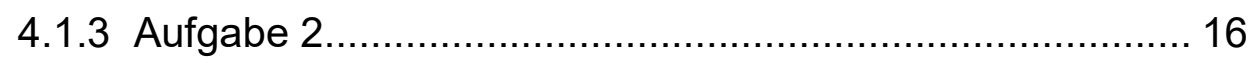

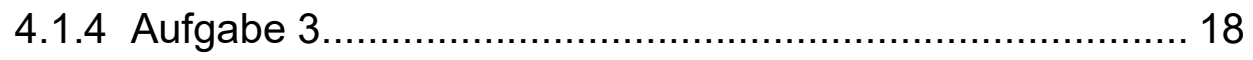

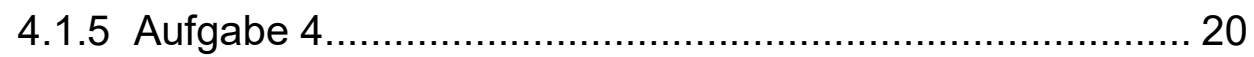

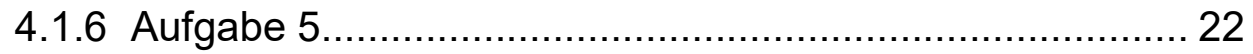

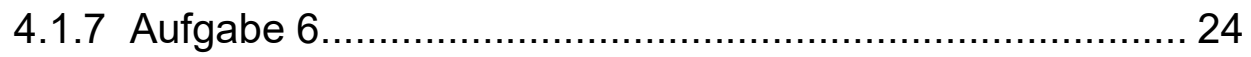

4.2 Auswertung der persönlichen Fragebögen................................ 26

4.2.1 Zeitmanagement und Kommunikation.........................26

4.2.2 Informationsfluss und Digitalisierung im klinischen Alltag.. 26

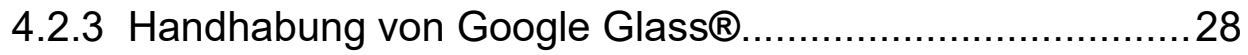

4.3 Zusammenfassung der Ergebnisse..........................................29 


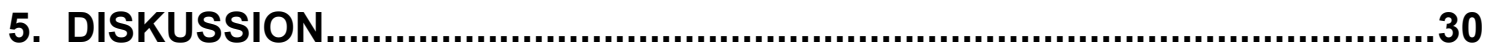

5.1 Benutzung von Google Glass ${ }^{\circledR}$ und technische Beobachtungen.....31

5.1.1 Allgemeine Handhabung und Tragekomfort......................31

5.1.2 Betriebs- und Akkulaufzeit...................................................

5.1.3 Custom Glass App und Sprachsteuerung....................... 32

5.1.4 Bildqualität und Beeinflussung des Sichtfeldes................. 33

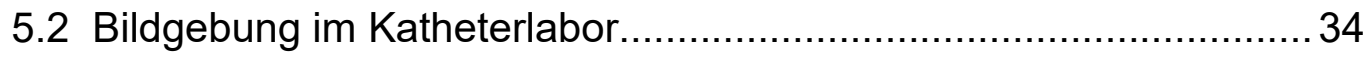

5.2.1 Visuelle Wahrnehmung und Verarbeitung..........................35

5.2.2 Ablenkung durch visuelle Eindrücke...................................35

5.3 Analyse der Aufgabenauswertung............................................... 37

5.3.1 Visuelle Fokussierung und Autonomie............................. 37

5.3.2 Bildauflösung, Bildkontrastierung und Farbsättigung......... 38

5.3.3 Selektive Aufmerksamkeit............................................. 39

5.3.4 Komplexität visueller Informationen...................................39

5.4 Ausblick auf heutige Systeme.................................................... 40

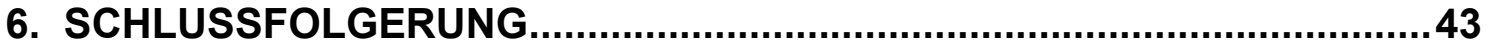

7. FAZIT

LITERATURVERZEICHNIS...................................................................

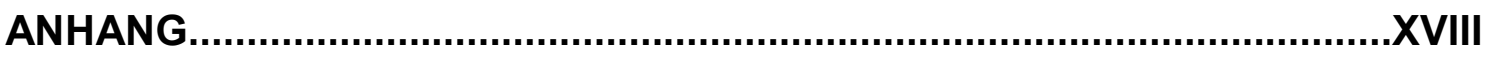

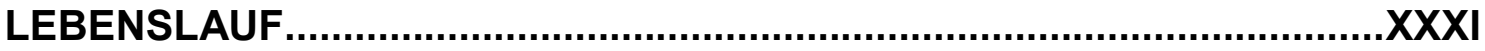

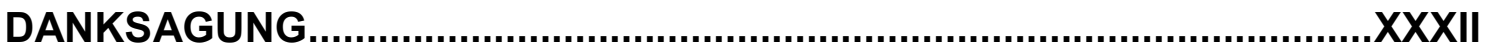

SCHRIFTLICHE ERKLÄRUNG...........................................................XXXIV 


\section{ABKÜRZUNGSVERZEICHNIS}

A.

Abb.

$\mathrm{ABI}$

Anh.

AR

Aufg.

BMI

caud.

DSA

engl.

fT3 / fT4

GG

$\mathrm{HbA1c}$

HDL

$\mathrm{HF}$

HKL

ID

LDL

LLC

Ltd.

Min.

neg.

oHMD

pAVK

PTA

RAO

$\mathrm{RR}$

Sek.

Tab.

TSH

VR
Arteria

Abbildung

Ankle-Brachial Index

Anhang

Augmented Reality

Aufgabe

Body Mass Index

kaudal

Digitale Subtraktionsangiografie

englisch

freies Trijodthyronin / freies Thyroxin

Google Glass $®$ Explorer Edition

Hämoglobin A1C

high-density Lipoprotein

Herzfrequenz

(Herz-)Katheterlabor

Identifikationsnummer

low-density Lipoprotein

Limited Liability Company

Limited (Company)

Minuten

negativ

optical Head-Mounted Display

periphere arterielle Verschlusskrankheit

Perkutane transluminale Angioplastie

right anterior oblique

Blutdruck

Sekunden

Tabelle

Thyreoidea-stimulierendes Hormon (Thyreotropin)

Virtual Reality 


\section{ABBILDUNGS- UND TABELLENVERZEICHNIS}

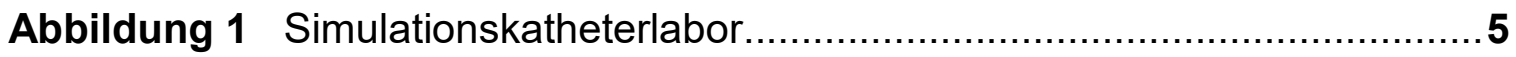

Abbildung 2 PTA-Simulation mit Benutzung von Google Glass ${ }^{\circledR}$ (Gruppe B)... 5

Abbildung 3 Google Glass $₫$ Explorer Edition........................................... 6

Abbildung 4 Beispielbild des Hauptmenüs der Custom Glass App..................8

Abbildung 5 Hardware des ANGIO-Mentor ${ }^{\circledR}$ Simulators................................ 9

Abbildung 6 Vergleich der Gesamtpunktzahl beider Testgruppen.................11

Abbildung 7 Simulationszeit im Gruppenvergleich...................................... 12

Abbildung 8 Zeitverlust durch Neustarts der Custom Glass App................... 12

Abbildung 9 Simulationszeit abzüglich der App-Neustarts in Gruppe B...........13

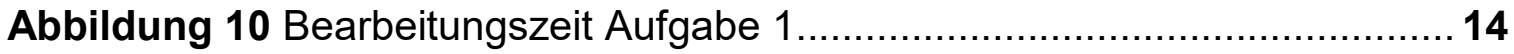

Abbildung 11 Bearbeitungszeit Aufgabe 1 abzüglich der App-Neustarts...........15

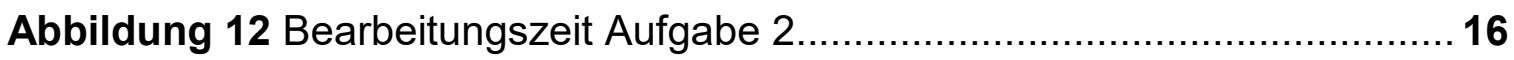

Abbildung 13 Bearbeitungszeit Aufgabe 2 abzüglich der App-Neustarts...........17

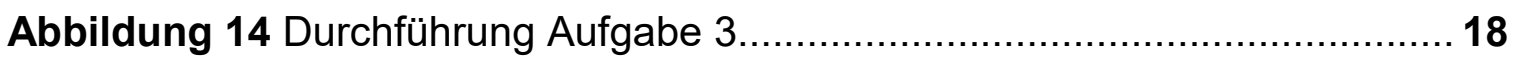

Abbildung 15 Bearbeitungszeit Aufgabe 3 ................................................ 19

Abbildung 16 Bearbeitungszeit Aufgabe 3 abzüglich der App-Neustarts...........19

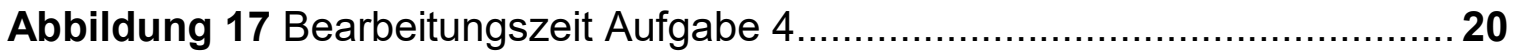

Abbildung 18 Bearbeitungszeit Aufgabe 4 abzüglich der App-Neustarts...........21

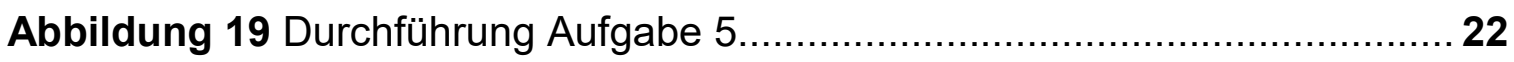

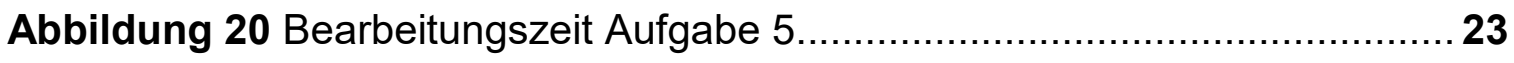

Abbildung 21 Bearbeitungszeit Aufgabe 5 abzüglich der App-Neustarts...........23

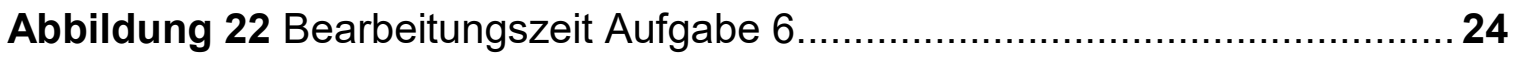

Abbildung 23 Bearbeitungszeit Aufgabe 6 abzüglich der App-Neustarts...........25

Abbildung 24 Informationsgewinn aus digitalen gegenüber analogen Medien...27

Abbildung 25 Informationstransfer in digitaler gegenüber analoger Form..........27

Abbildung 26 Einfluss auf das Sichtfeld bei der Benutzung von Google Glass $₫ 28$

Abbildung 27 Bildqualität der Grafiken bei der Benutzung von Google Glass $₫ . .29$

Abbildung 28 Sprachsteuerung bei der Benutzung von Google Glass $₫$.............29

Tabelle 1 Checkpoints und Aufgabenstellung.............................................

Tabelle 2 Technische Details zu Google Glass $₫$...................................... 7 


\section{GENDER-HINWEIS}

In dieser Arbeit wird aus Gründen der besseren Lesbarkeit das generische Maskulinum verwendet. Weibliche und diverse Geschlechteridentitäten werden dabei ausdrücklich mitgemeint, soweit es für die Aussage erforderlich ist. 


\section{ZUSAMMENFASSUNG}

Hintergrund. Ziel dieser Studie war es, zu bewerten, ob die Datenübertragung während peripherer endovaskulärer Eingriffe durch ein sprachgesteuertes, optisches Head-Mounted Display verwirklicht, und ob hierdurch der Arbeitsablauf der Intervention verbessert werden kann.

Methoden. Wir benutzten die Google Glass $₫$ Explorer Edition in Verbindung mit einer eigens entwickelten Glass App, um vorhandene Grafiken über die Datenbrille durch Sprachbefehle zugänglich zu machen. 40 Medizinstudenten im letzten Drittel des Medizinstudiums wurden in zwei Gruppen randomisiert.

Jeder Proband erhielt die Aufgabe eine PTA der A. femoralis superficialis an einem High-Fidelity-VR-Simulator (ANGIO-Mentor ${ }^{\circledR}, \quad 3 D$ Systems) durchzuführen. Während Gruppe A hierfür nötige Informationen über einen zusätzlich installierten Monitor erhielt, verwendete Gruppe B Google Glass $₫$, um jeweilige Informationen durch zuvor definierte Sprachbefehle aufzurufen. Die objektive Bewertung der erbrachten Leistung erfolgte durch standardisierte Bewertungsbögen in dichotomer Nominalskalierung und durch die Messung der für die Aufgaben benötigten Zeit. Am Ende jeder Simulation erfolgte die subjektive Bewertung seitens der Probanden durch standardisierte Fragebögen mit 5-Level-Likert-Skalierung.

Ergebnisse. Eine maximale Punktzahl von 10 Punkten war erreichbar. Der in Gruppe A und Gruppe B gefundene Median lag bei 9 Punkten mit nicht signifikanten Abweichungen $(p=0,91)$. Die Gesamtdauer des Eingriffs betrug zwischen 12 und 14 Minuten. Gruppe B war unter Verwendung von Google Glass $₫$, aufgrund technischer Schwierigkeiten mit der getesteten App, im Schnitt um 1:07 Minuten signifikant langsamer $(p=0,01)$. Dennoch konnte nachgewiesen werden, dass Google Glass $®$ bei dem Transfer einfacher Informationen schneller oder zumindest gleichwertig gegenüber dem klassischen Monitoring war. 
In diesem Kontext erachteten 92,5\% der Probanden die Digitalisierung im klinischen Alltag als sinnvoll. 17 von 20 Teilnehmern (85\%) empfanden die Handhabung von Google Glass $®$ als einfach bis sehr einfach. Alle Teilnehmer waren der Ansicht, dass Augmented Reality bei peripheren endovaskulären Eingriffen im Katheterlabor nützlich sein könnte.

Schlussfolgerung. Google Glass ${ }^{\circledR}$ war dem klassischen Monitoring im Katheterlabor hinsichtlich der Gesamtinterventionszeit nur geringfügig unterlegen und behinderte den Arbeitsablauf während einer simulierten PTA der A. femoralis superficialis nicht. Unsere Studie offenbarte hierbei technische Schwierigkeiten bei der Genauigkeit der Spracherkennung und der Bildqualität von Google Glass ${ }^{\circledR}$. Trotzdem konnten einzelne Aufgaben durch die Nutzung der Google Glass $®$ signifikant schneller durchgeführt werden. Wir erwarten, dass nach Überwindung dieser technischen Probleme der Arbeitsablauf während endovaskulären Eingriffen mit einem optischen Head-Mounted Display verbessert werden kann. 


\section{ABSTRACT}

Background. The intention of this study was to evaluate whether data transmission during peripheral endovascular interventions can be realized by a voice controlled optical head mounted display and to see whether it improves the overall procedural performance.

Methods. We used the Google Glass ${ }^{\circledR}$ Explorer Edition in conjunction with a custom Glass App, making preexisting imaging files accessible by voice commands. 40 medical students in their 5 th year of medical school were randomized into two groups.

Each subject got the task to perform a PTA of the superficial femoral artery on a high- fidelity-VR-simulator (ANGIO-Mentor ${ }^{\circledR}$, 3D Systems). While Group A obtained typical information from an additional monitor, Group B used Google Glass $®$ to bring the particular information to their attention by a special voice command. The objective evaluation was carried out by using a standardized evaluation protocol including the performance in dichotomous nominal scale and the required time for each task. At the end of the simulation each participant had to fill in a questionnaire using a typical 5-level Likert scaling for subjective evalution.

Results. A maximum score of 10 points was achievable. The median found in Group A and Group B was at 9 points with non-significant deviations $(p=0,91)$. The individual procedure time was between 12 and 14 minutes. Group B using Google Glass $₫$ was significantly slower by $1: 07$ minutes $(p=0,01)$ on average mostly due to technical difficulties with the prototype App. Focusing on the transfer of simple information our study proved Google Glass $®$ to be faster or at least equivalent compared to a conventional setting of monitors. In this context $92.5 \%$ considered the digitization of daily clinical practice as useful. 17 of 20 participants $(85 \%)$ perceived the handling of Google Glass ${ }^{\circledR}$ as easy to very easy. All participants shared the opinion that augmented reality could be useful during peripheral interventions in the cathlab. 
Conclusions. Google Glass ${ }^{\circledR}$ was only marginally inferior to conventional catheter laboratory monitoring with regard to procedural time and did not impede workflow during a simulated PTA of the superficial femoral artery. Moreover, some tasks were performed significantly faster by using Google Glass $₫$. Our study revealed technical difficulties with the accuracy of voice recognition and quality of imaging on Google Glass $₫$. We expect that after overcoming those technical problems, cathlab workflow during endovascular interventions could be improved by using an optical head mounted display. 


\section{EINLEITUNG}

Aufgrund der rasenden Entwicklung moderner Technologien und damit einhergehenden innovativen Produkten, befinden wir uns zurzeit in einer Phase des digitalen Zeitalters, in welcher sich der Informationstransfer nicht mehr lediglich über die schnellstmögliche Übermittlung von Daten in den verschiedensten Formaten definiert. Zunehmend spielt der unkomplizierte und intuitive Erhalt gewünschter Informationen unabhängig von Zeit und Ort eine führende Rolle (1).

Vor diesem Hintergrund besteht heute die Möglichkeit, unsere Wahrnehmung der Umgebung digital zu ergänzen (Augmented Reality, AR) oder eine vollständig digitale, künstliche Umgebung (Virtual Reality, VR) zu schaffen (2). Das Thema VR konnte unter anderem durch die Einführung von optischen Head-Mounted Displays (oHMD) etabliert werden.

Während oHMD bereits mit Oculus Rift巴 (3), HTC Vive® (4) oder PlayStation $V R \circledast(5)$ in der Unterhaltungsindustrie Einzug gehalten haben, beginnt diese Technologie ebenfalls in der Medizin Fuß zu fassen. Hier besteht der Nutzen darin, klinische Abläufe, wie zum Beispiel die Dokumentation von Patienteninformationen (6), zu vereinfachen und hierdurch Zeit einzusparen.

Ziel dieser Studie war es, die Funktionalität eines oHMD in den Arbeitsablauf einer perkutanen transluminalen Angioplastie (PTA) der A. femoralis superficialis zu integrieren und herauszufinden, ob hierdurch eine Verbesserung des Arbeitsablaufs erreicht werden kann. Hierfür verwendeten wir die Google Glass $®$ Explorer Edition (GG) - einen als Brille tragbaren Miniatur-Computer, der 2014 von dem US-amerikanischen Unternehmen Google LLC veröffentlicht wurde. GG macht es möglich, visuelle Informationen mithilfe eines Glasprismas auf das periphere Sichtfeld des Benutzers zu projizieren und akustische Signale per Knochenleitung zu übertragen. 
Durch eine benutzerdefinierte Glass App (Custom Glass App) können die verschiedenen Funktionen von GG ergänzt werden. Beispielsweise lassen sich Bilder über Sprachbefehle anzeigen, was eine wichtige Voraussetzung darstellt, um solch ein oHMD in einem sterilen Arbeitsumfeld wie einem Operationssaal zu verwenden, da die Bedienung hier nicht per Handsteuerung erfolgen kann.

Gegenstand unserer Studie war nicht die Beurteilung der korrekten Durchführung der Intervention, sondern die Beurteilung des Arbeitsablaufes sowie die Erfassung des subjektiven Untersuchereindrucks. Es wurden daher zwei Varianten der visuellen Informationsübertragung während einer am VR-Simulator dargestellten PTA verglichen. 


\section{PROBANDEN, MATERIAL UND METHODEN}

Jeder Teilnehmer erhielt eine grundlegende, inhaltliche Einweisung in periphere endovaskuläre Interventionen durch einen, eigens für die Studie aufgenommenen, Podcast sowie eine standardisierte technische Einweisung zur Benutzung des High-Fidelity-VR-Simulators. Das Einverständnis jedes Teilnehmers zur Verwendung der anonymisierten personenbezogenen Daten wurde schriftlich eingeholt (Anh.1).

\subsection{Studiendesign}

Alle Probanden erhielten die Anweisung, im Rahmen der simulierten PTA einer hochgradigen Stenose der A. femoralis superficialis, insgesamt sechs fallbezogene Aufgaben zu bearbeiten (Tab.1). Diese wurden an determinierten Checkpoints gestellt und entweder ohne (Kontrollgruppe: Gruppe A) oder unter Zuhilfenahme von GG (Experimentalgruppe: Gruppe B) beantwortet.

Tabelle 1 Checkpoints und Aufgabenstellung

\begin{tabular}{|c|c|}
\hline Checkpoint & Aufgabe \\
\hline 1. Vor Interventionsbeginn & $\begin{array}{l}\text { "Lesen Sie die vorliegende Anamnese } \\
\text { und stellen Sie eine Verdachtsdiagnose" }\end{array}$ \\
\hline 2. Platzierung der Schleuse & $\begin{array}{l}\text { "Lokalisieren Sie die Pathologie in der } \\
\text { vorliegenden Angiografie" }\end{array}$ \\
\hline 3. Darstellung der Stenose & $\begin{array}{l}\text { "Beurteilen Sie vorliegende Laborwerte } \\
\text { in Hinsicht auf relevante Parameter" }\end{array}$ \\
\hline 4. Vor Stentimplantation & $\begin{array}{l}\text { "Überprüfen Sie das Monitoring auf } \\
\text { kritische Vitalparameter" }\end{array}$ \\
\hline 5. Nach Stentimplantation & $\begin{array}{l}\text { "Kontrollieren und bewerten Sie die } \\
\text { postinterventionelle Fluoroskopie" }\end{array}$ \\
\hline 6. Nach Interventionsende & $\begin{array}{l}\text { "Bewerten Sie die Fotodokumentation } \\
\text { der Füße Ihres Patienten" }\end{array}$ \\
\hline
\end{tabular}


Die objektive Bewertung der durchgeführten Testungen wurde dann durch den Studienleiter unter Verwendung von standardisierten Bewertungsprotokollen durchgeführt (Anh.2). Diese dokumentierten die Durchführung der Aufgaben mittels dichotomer Nominalskala sowie die jeweils erforderliche Zeit (4.1). Zudem wurde bei der Verwendung von GG zusätzlich die Anzahl etwaiger Neustarts der App beziehungsweise der dadurch entstandene Zeitverlust notiert.

Am Ende eines jeden Testdurchlaufs erfolgte die Dokumentation der subjektiven Einschätzung jedes Probanden zur Thematik anhand eines Fragebogens im Stil einer typischen 5-Level-Likert-Skala (Anh.3). Hierbei wurden vor allem die Elemente Kommunikation, Ablenkung, Zeitmanagement und verschiedene Arten des Informationsaustauschs aufgegriffen (4.2).

Gruppe B erhielt zusätzlich spezifische Fragen zur Verwendung von GG (Anh.4).

Für freie Bemerkungen, Anregungen oder Kritik seitens der Probanden wurde ein Volltext-Kommentarfeld auf den Fragebögen eingefügt.

\subsection{Probandenkollektiv}

40 Studenten im fünften und sechsten Jahr des Medizinstudiums wurden rekrutiert und durch eine computergenerierte Randomisierungsliste gleichmäßig in zwei Gruppen aufgeteilt. In einer Altersspanne von 20 bis 30 Jahren lag das Durchschnittsalter der Probanden bei 24,4 Jahren. Studenten mit Sehstörungen wurden aus Gründen der Durchführbarkeit von unserer Studie ausgeschlossen. Neben der klassischen Anordnung von Bildschirmen im Simulationskatheterlabor (Abb.1) - bestehend aus einem Live-AngiografieMonitor und einem Referenz-Monitor - erhielt Gruppe A einen weiteren Monitor, welcher unmittelbar rechts neben der oben genannten Anordnung platziert wurde (Abb.1, Pfeil). Dieser bildete die nötigen Informationen zur Bearbeitung der gestellten Aufgaben ab. Gruppe B im Gegenzug führte die PTA mit Hilfe von GG durch und konnte sich die nötigen Informationen per Sprachsteuerung auf dem oHMD zugänglich machen (Abb.2). 
Abbildung 1 Simulationskatheterlabor

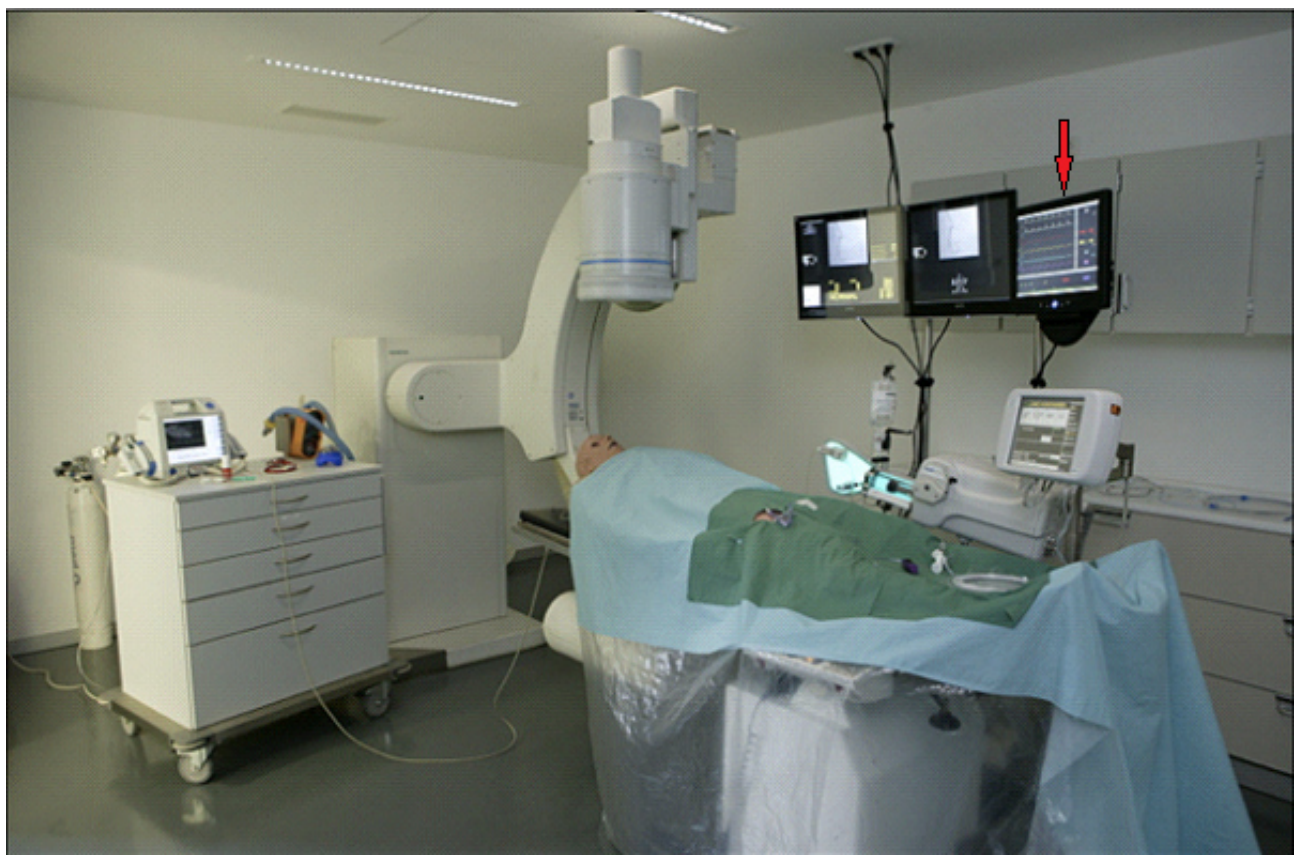

"Pfeil" = Zusätzlicher Monitor für Gruppe A

Abbildung 2 PTA-Simulation mit Benutzung von Google Glass ${ }^{\circledR}$ (Gruppe B)

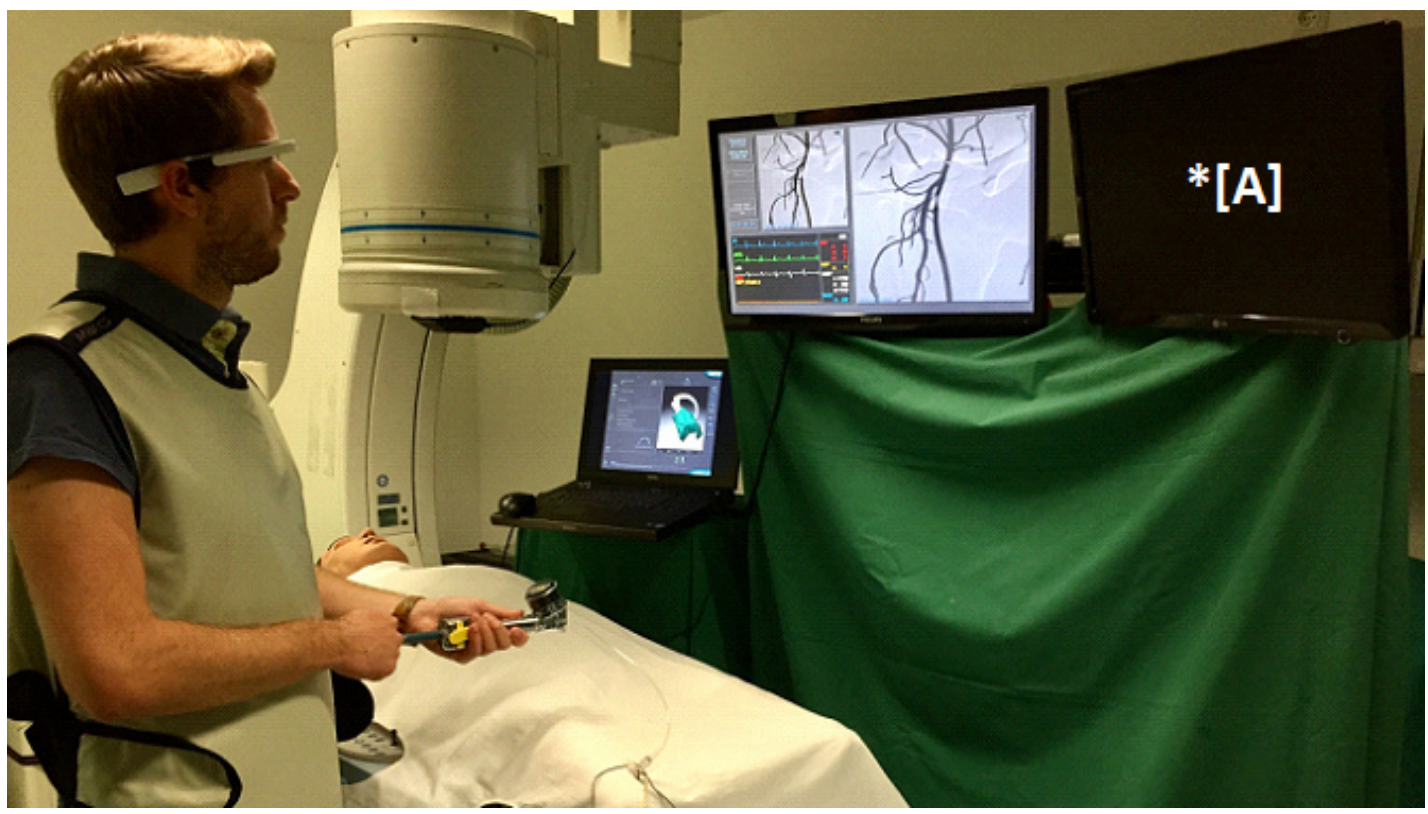

${ }^{*}[\mathrm{~A}]=$ Zusätzlicher Monitor für Gruppe A 


\subsection{Google Glass $®$ Explorer Edition}

Die GG Explorer Edition ( $\underline{\mathrm{Abb} .3}$ ) ist ein sogenanntes optisches Head-Mounted Display (oHMD), das seitens des Herstellers nur einer begrenzten Anzahl von Nutzern zu Testzwecken zur Verfügung gestellt wurde. Es handelt sich hierbei um einen am Kopf tragbaren Computer, welcher dem Benutzer mit Hilfe eines Glasprismas, das oberhalb des rechten Auges montiert ist, einen virtuellen Bildschirm in den rechten oberen Quadranten des Sichtfelds projiziert. Der Mikroprozessor mit integriertem Touchpad befindet sich hierbei ebenfalls auf der rechten Seite, auf Höhe der Schläfe des Benutzers, mit welchem die manuelle Navigation durch die Software von GG gewährleistet ist. Zudem ist die Benutzung des Gerätes durch ein integriertes Mikrofon auch per Sprachsteuerung möglich. Der Benutzer ist demnach durch GG in der Lage, die natürliche Umgebung für sich digital zu erweitern und mit der zusätzlichen visuellen Information zu interagieren, was dem Prinzip der AR entspricht. Weitere technische Details sind in Tabelle 2 aufgeführt.

Abbildung 3 Google Glass $₫$ Explorer Edition

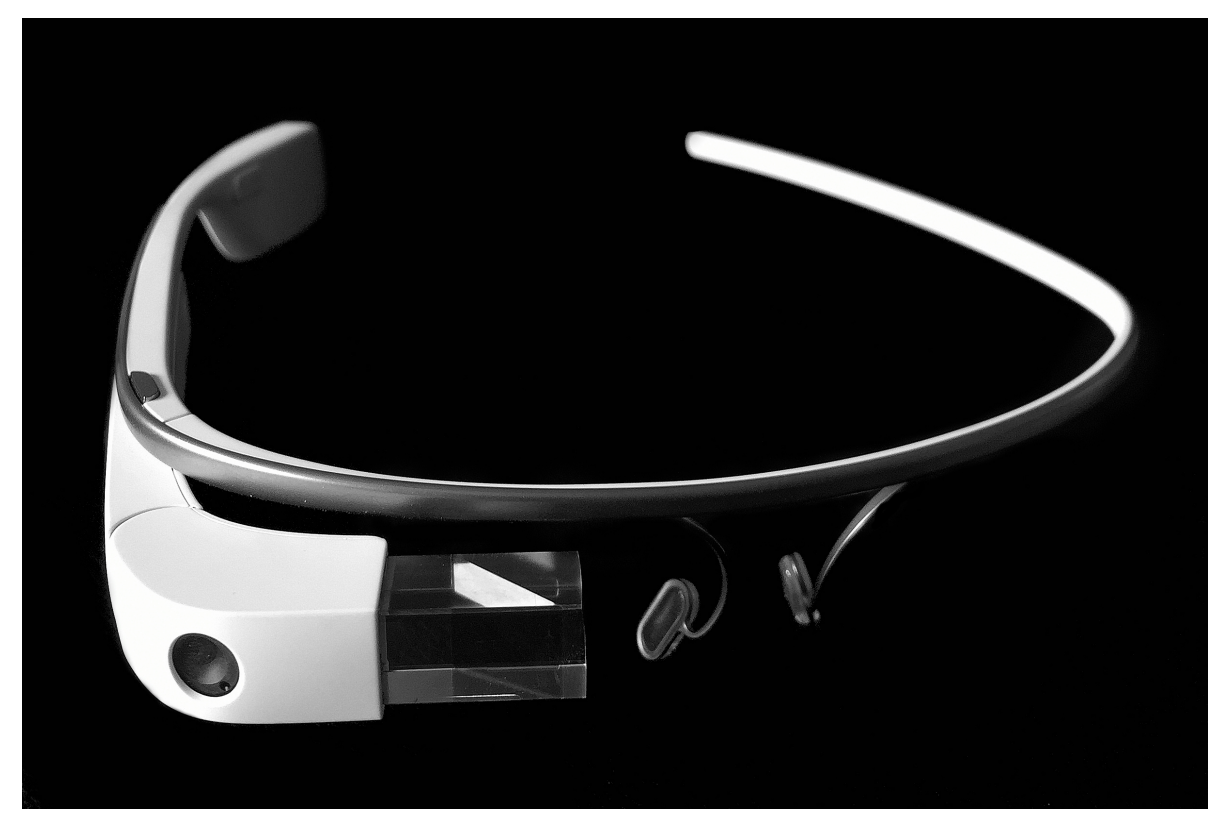


Tabelle 2 Technische Details zu Google Glass ${ }^{\circledR}(7,8)$

\begin{tabular}{ll}
\hline $\begin{array}{l}\text { Name } \\
\text { Entwickler } \\
\text { Hersteller }\end{array}$ & Google Glass $₫$ Explorer Edition \\
& Google LLC \\
Foxconn & \\
Betriebssystem & Glass OS (Google XE) \\
Prozessor & OMAP 4430 SoC, Dual-Core \\
Speicher & 16 GB Flash (12 GB verwendbar) \\
Anzeige & Prismaprojektor, 640×360 Pixel \\
Audio & Knochenleitungsüberträger \\
Kamera & Fotos 5 Mpx, Videos 720 px \\
Konnektivität & Wi-Fi 802.11 b/g, Bluetooth, micro-USB \\
Akku & $570 \mathrm{mAh}$ Lithiumionen-Batterie \\
Akkulaufzeit & $8 \mathrm{~h}$ (laut Hersteller) \\
Gewicht & $36 \mathrm{~g}$ \\
\hline
\end{tabular}

\subsection{Custom Glass App}

Die Custom Glass App ist eine im Rahmen der Studie speziell für GG entwickelte Anwendung, die den Zugriff auf im System hinterlegte Bilddateien ausschließlich durch Sprachsteuerung ermöglicht.

Die Idee zur Entwicklung dieser App war, GG in Katheterlaboren und Operationssälen, unter Verwendung der Spracherkennung, nutzbar zu machen. Eine manuelle Interaktion mit GG ist, aufgrund der Sterilitätsrichtlinien in eben jenen Bereichen, nicht möglich. Die jeweiligen Grafiken mit den korrespondierenden Sprachbefehlen wurden zuvor im Kontext der Studie festgelegt und in die App integriert.

Nach Aktivierung von GG kann die Custom Glass App gestartet werden, worauf zunächst automatisch die Spracherkennungsfunktion der Software initialisiert wird. Darauf fordert die App mit den abgebildeten Worten „To start demo say 'show me' " zur erstmaligen verbalen Interaktion auf. Die Anzeige wechselt in das Hauptmenü der Custom Glass App mit einer Auflistung der aktuell verfügbaren Sprachbefehle auf der linken Seite der GG-Projektion. Die zugehörigen Grafiken hingegen werden bei Aufruf der Bilder per Sprachbefehl auf der rechten Seite angezeigt. Die grafische Benutzeroberfläche der Custom Glass App ist exemplarisch in Abbildung 4 zu sehen. 
Abbildung 4 Beispielbild des Hauptmenüs der Custom Glass App

\section{Glass-App}

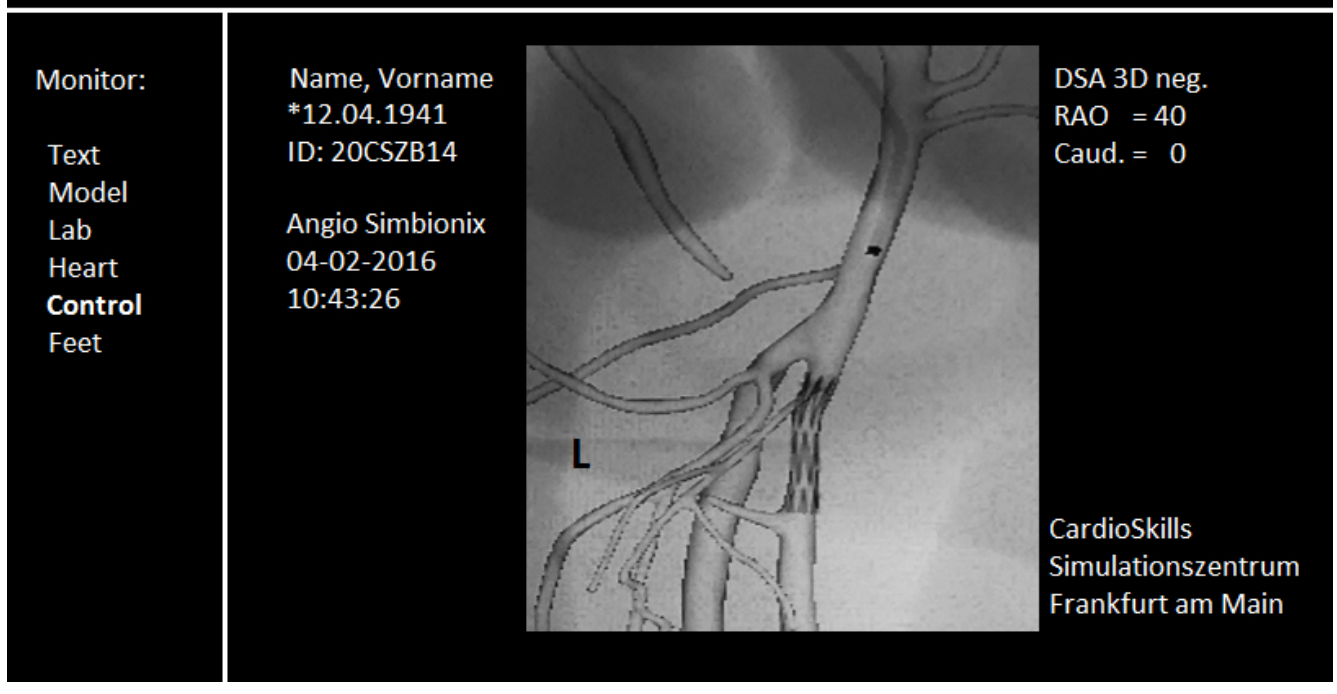

\subsection{ANGIO-Mentor}

Der ANGIO-Mentor ${ }^{\circledR}(\underline{A b b .5})$ ist ein mobiler High-Fidelity-VR-Simulator für endovaskuläre Katheterinterventionen, hergestellt von 3D Systems - ehemals Simbionix Ltd. (Airport City, Israel) (6). Der Simulator ermöglicht die Durchführung verschiedener diagnostischer oder interventioneller endovaskulärer Eingriffe. Hierbei ist das gleichzeitige Einführen von bis zu drei verschiedenen, frei wählbaren Drähten und Kathetern über das integrierte Tracking-Modul der Hardware möglich. Die jeweiligen Katheter beziehungsweise Drähte werden dafür im Menü des Simulators mit ihren spezifischen Eigenschaften, wie Größe, Material oder Biegung, durch den Benutzer ausgewählt. Darauf bildet der ANGIO-Mentor ${ }^{\circledR}$ die jeweiligen Devices über den Angiografie-Monitor ab und simuliert deren Eigenschaften durch ein dynamisches Force-Feedback-System. Dem Benutzer wird so ein realitätsnahes Gefühl mit immersivem Simulationserlebnis ermöglicht. 
Abbildung 5 Hardware des ANGIO-Mentor ${ }^{\circledR}$ Simulators

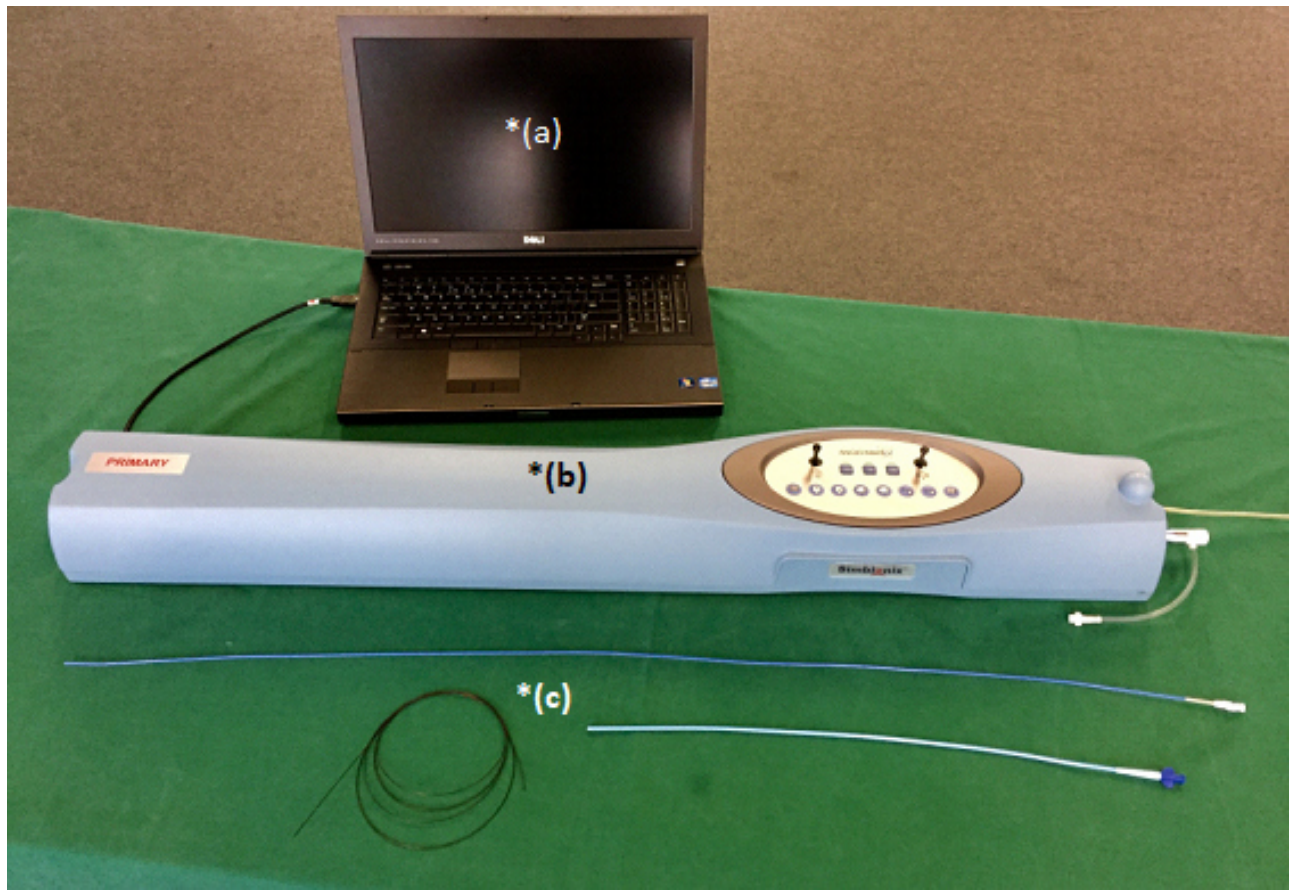

*(a) Laptop (Software bzw. Abbildung des Hauptmenüs)

*(b) Tracking-Modul mit Force-Feedback-System

*(c) Katheter und Drähte

\subsection{Statistik}

Alle gesammelten Daten wurden mit Hilfe des Programms BiAS (Version 10.0 für Windows) und Microsoft Excel 2002 (Version 10 für Windows) statistisch analysiert und grafisch aufbereitet. Zur Überprüfung der Normalverteilung der gemessenen Zeiten, wurde hierbei der Kolmogorov-Smirnov-Lilliefors-Test angewendet. Bei parametrischer Verteilung wurde die Signifikanz mit dem Zwei-Stichproben-t-Test überprüft. Die Überprüfung der Signifikanz der erreichten Gesamtpunktzahlen erfolgte bei nicht-parametrischer Verteilung mit Hilfe des Wilcoxon-Mann-Whitney-U-Tests. Die Signifikanz der Unterschiede zwischen beiden Gruppen bei der Durchführung der einzelnen Aufgaben, wurde bei diskreten Variablen mit dem Chi-Quadrat-Test überprüft. 


\section{FRAGESTELLUNG}

Anhand der gesammelten Daten sollten die folgenden Fragen geklärt werden:

a) Kann GG bei einer peripheren endovaskulären Katheterintervention hilfreich sein?

b) Ist die Custom Glass App während einer peripheren endovaskulären Katheterintervention zuverlässig einsetzbar?

c) Kann der Arbeitsablauf einer peripheren endovaskulären Katheterintervention durch die kombinierte Nutzung der Custom Glass App und GG verbessert werden? 


\section{ERGEBNISSE}

Der Einfluss der getesteten bildgebenden Verfahren auf den Arbeitsablauf der PTA wurde verglichen, indem die gemessenen Bearbeitungszeiten sowie die Bewertung der gestellten Aufgaben mittels Scoring-System durch standardisierte Bewertungsprotokolle dokumentiert wurden. Die Erfassung der subjektiven Ansichten und Meinungen der Probanden gegenüber der jeweiligen Bildgebung und zu den Kernthemen unserer Studie erfolgte im Anschluss der durchgeführten Simulation anhand von Fragebögen.

\subsection{Auswertung der Bewertungsprotokolle zur Simulation}

\subsubsection{Gesamtpunktzahl und Simulationszeit}

Die korrekte Lösung der gestellten Aufgaben im Rahmen der Simulation wurde mit einer maximalen Punktzahl von 10 bewertet (Anh.2). Der Median sowohl in Gruppe A (Kontrollgruppe) als auch in Gruppe B (Experimentalgruppe) lag bei insgesamt 9 Punkten ohne signifikante Abweichungen der beiden Gruppen $(p=0,91)(\underline{\text { Abb.6) }}$.

Abbildung 6 Vergleich der Gesamtpunktzahl beider Testgruppen

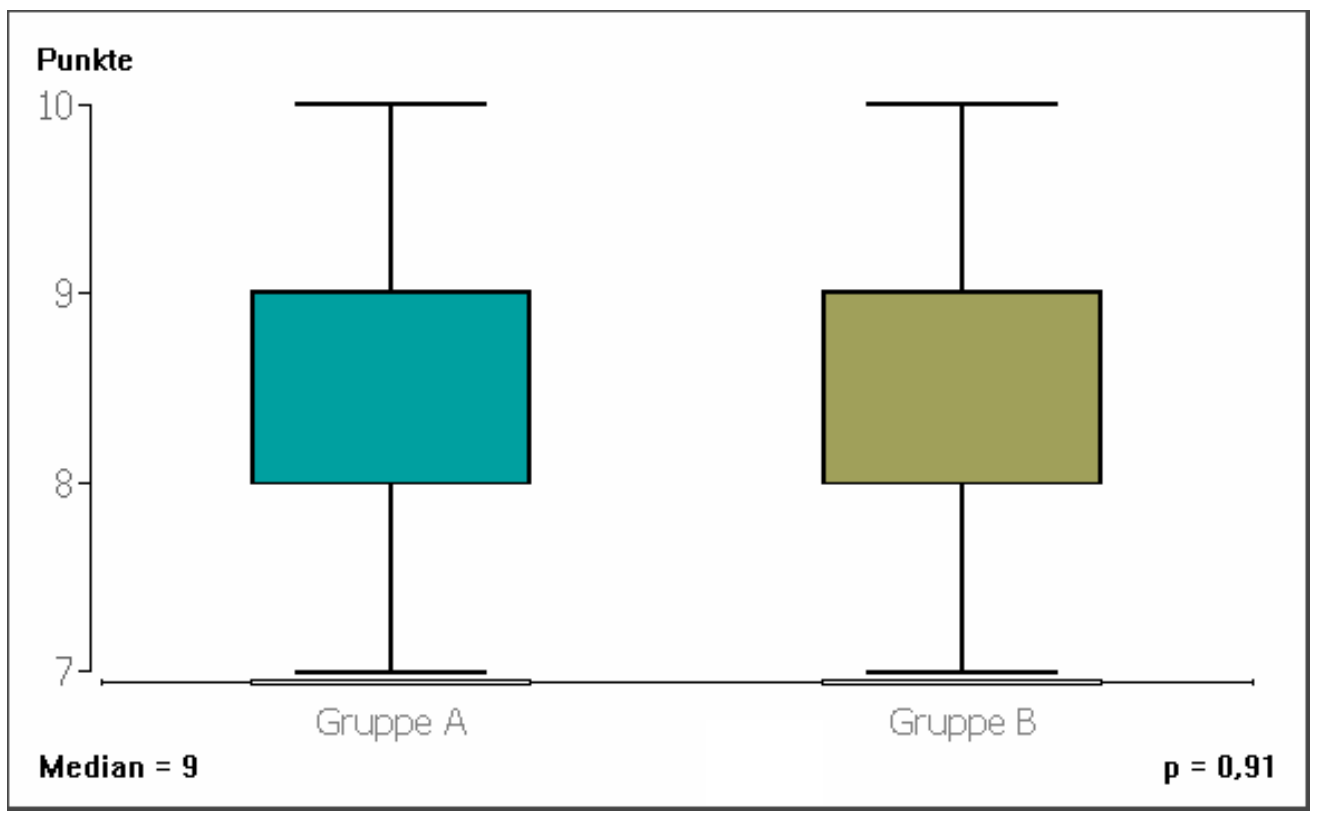


Die durchschnittlich benötigte Bearbeitungszeit für die simulierte PTA lag zwischen 12 und 14 Minuten (Median = 13:13 Minuten). Gruppe B war mit 13:49 Minuten signifikant langsamer $(p=0,01)$ als Gruppe A mit 12:43 Minuten (Abb.7). Hierbei benötigte Gruppe B aufgrund von technischen Herausforderungen mit GG im Schnitt neun bis zehn Neustarts der Custom Glass App, was in einer um 66,5 Sekunden (8,02\%) verlängerten Simulationszeit resultierte (Abb.8).

Abbildung 7 Simulationszeit im Gruppenvergleich

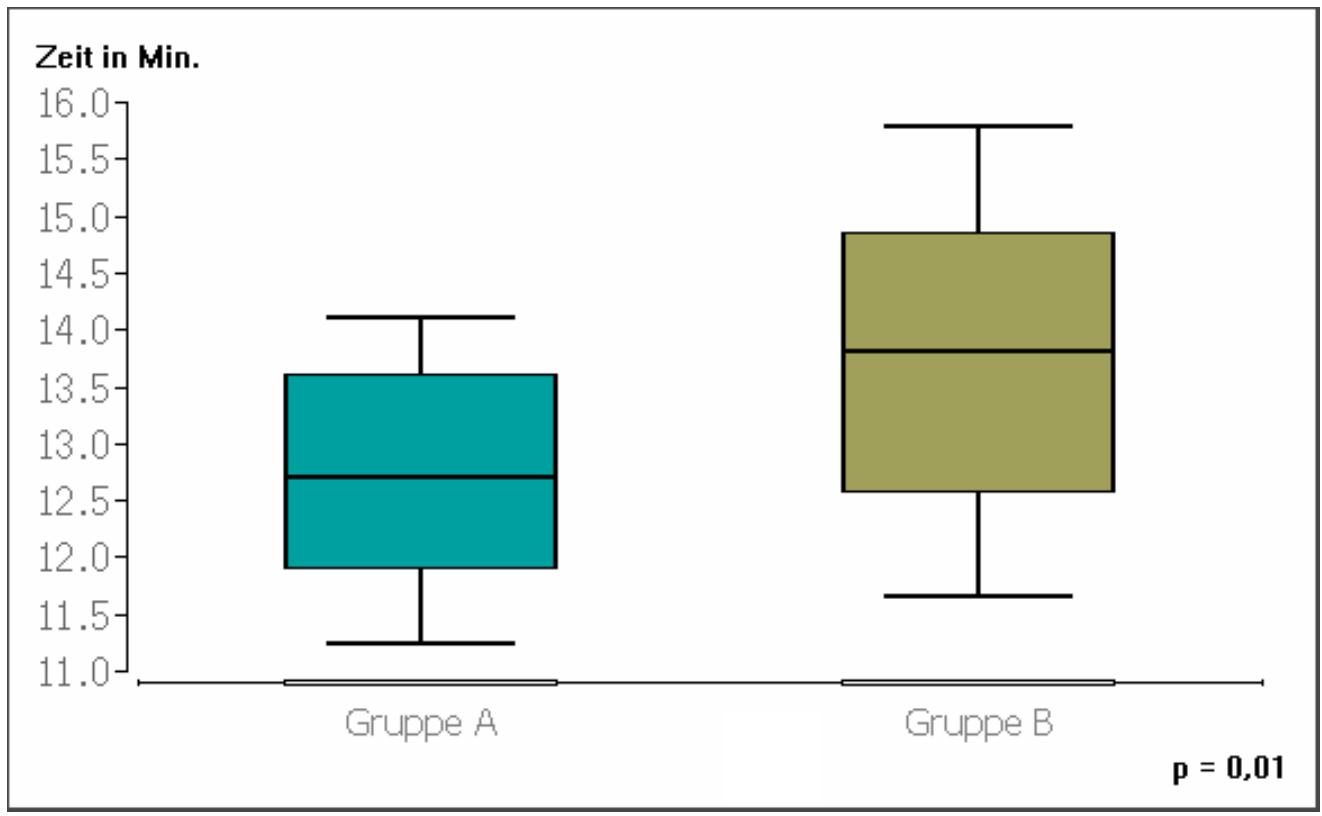

Abbildung 8 Zeitverlust durch Neustarts der Custom Glass App

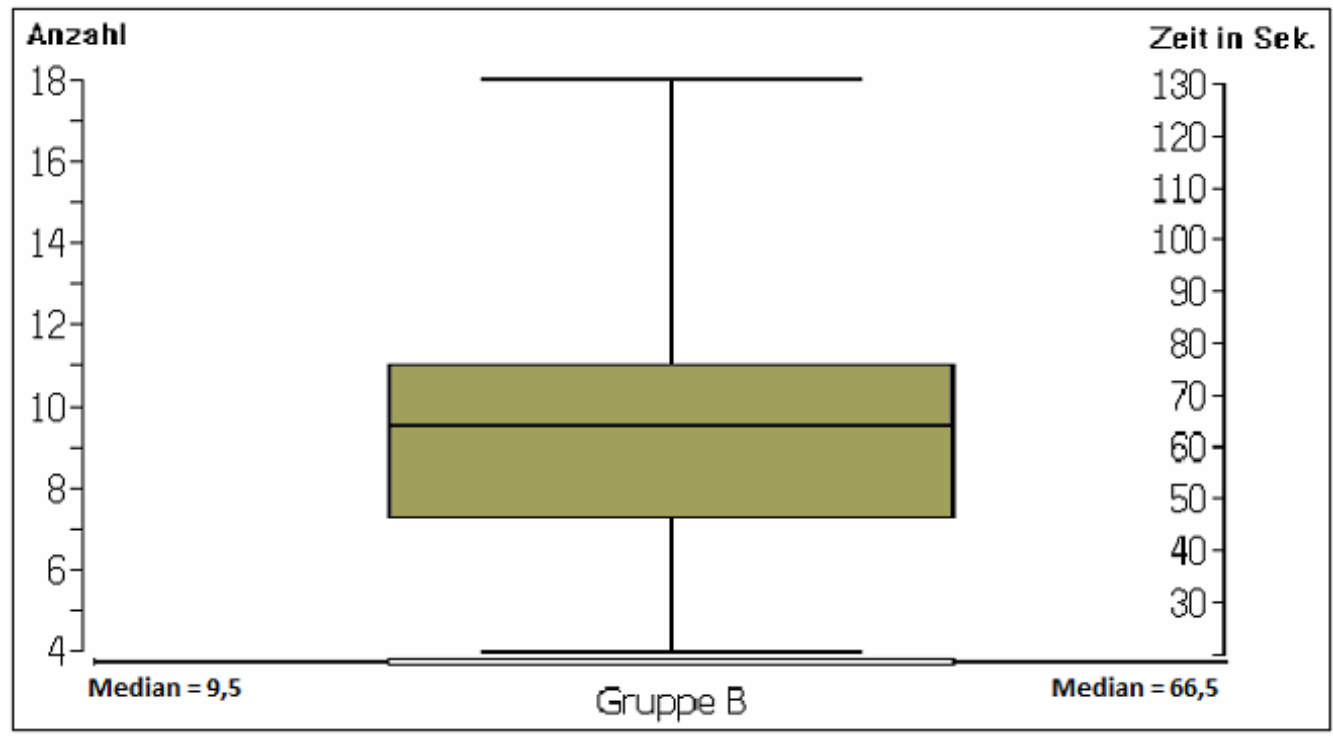


Wird der Zeitverlust durch die genannten Neustarts der Custom Glass App aus der reinen Bearbeitungszeit der Simulation durch Gruppe B herausgerechnet, so erhält man einen Median von 12:20 Minuten als durchschnittliche Gesamtzeit. In diesem Fall besteht kein signifikanter Zeitunterschied $(p=0,66)$ in der Durchführung des simulierten Fallbeispiels zwischen beiden Gruppen (Abb.9).

Abbildung 9 Simulationszeit abzüglich der App-Neustarts in Gruppe B

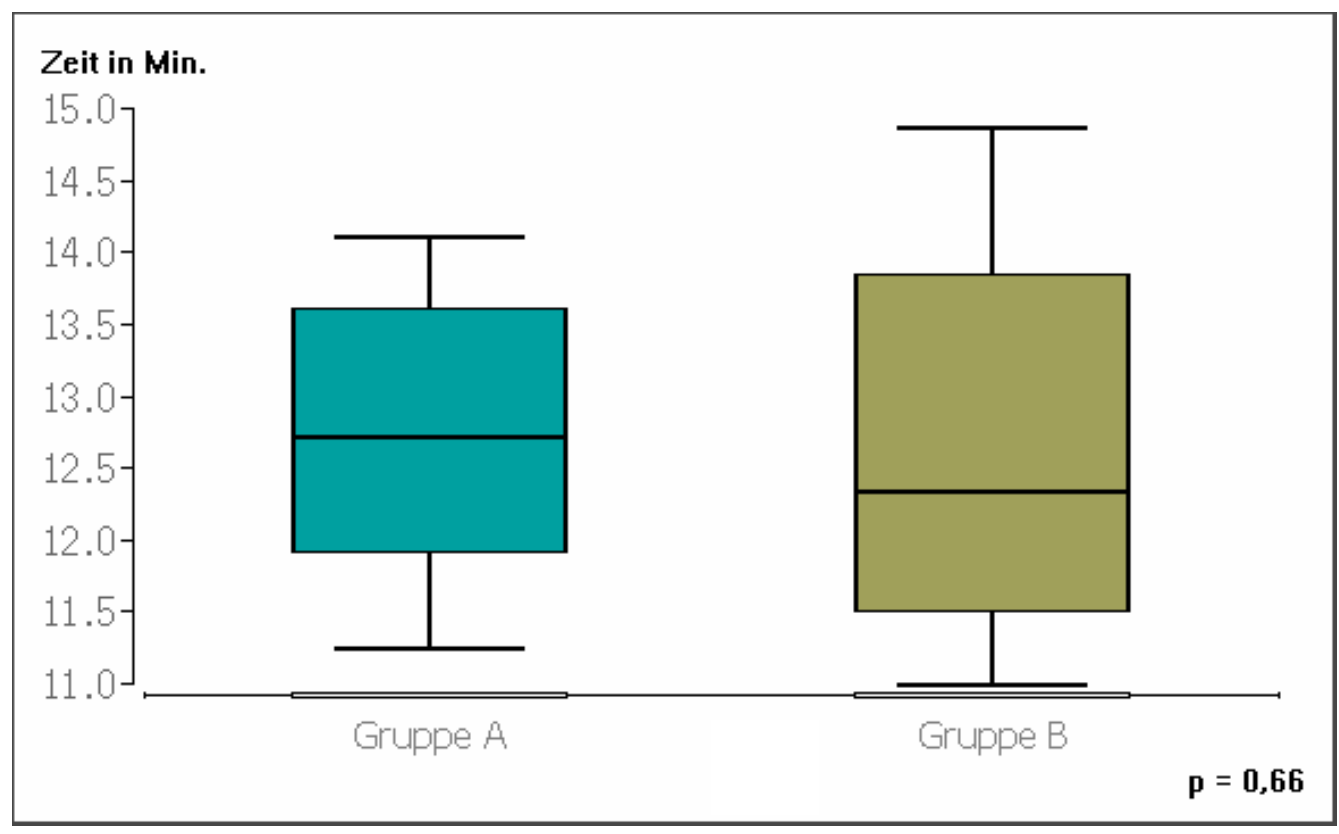

Um den zeitlichen Einfluss der App-Neustarts auf die erzielten Ergebnisse in Gruppe B zu quantifizieren und im weiteren Verlauf zu diskutieren, wurden im Folgenden zusätzlich zu den tatsächlich gemessenen Zeiten die Zeiten nach Abzug der App-Neustarts und die daraus resultierende Signifikanz angegeben. Diese Werte stellen keine Korrektur der eigentlichen Messung dar. Sie sollen lediglich ein Maß zur Orientierung sein, wie stark sich die Softwareproblematik zeitlich auf das gemessene Ergebnis ausgewirkt haben könnte. 


\subsubsection{Aufgabe 1}

"Lesen Sie die vorliegende Anamnese und stellen Sie eine Verdachtsdiagnose". Alle Probanden (100\%) kamen nach sorgfältigem Lesen der PatientenAnamnese und des klinischen Befundes (Anh.5) zu der korrekten Verdachtsdiagnose einer schweren, peripheren arteriellen Verschlusskrankheit (pAVK) des rechten Beines bei zugrunde liegendem, ausgeprägtem metabolischem Syndrom. Gruppe B war unter Nutzung von GG signifikant langsamer $(p=0,02)$ als Gruppe A mit dem klassischem Monitor-Setting im Simulationskatheterlabor (․ㅣb.10).

Abbildung 10 Bearbeitungszeit Aufgabe 1

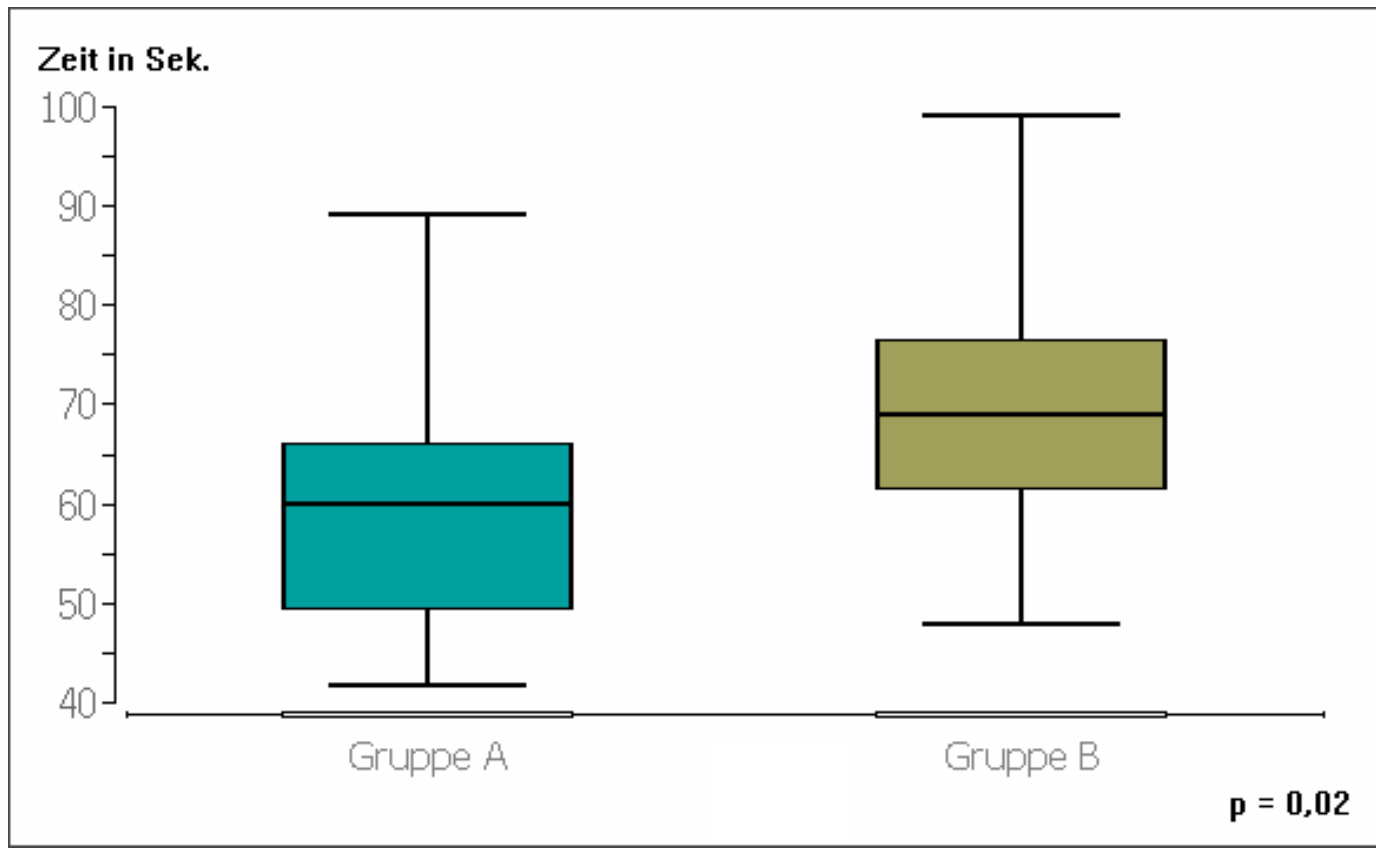


Abzüglich des Zeitverlustes durch die Neustarts der Custom Glass App, besteht kein signifikanter Unterschied ( $p=0,77$ ) hinsichtlich der benötigten Zeit zur Stellung der Verdachtsdiagnose zwischen beiden Gruppen (Abb.11).

Abbildung 11 Bearbeitungszeit Aufgabe 1 abzüglich der App-Neustarts

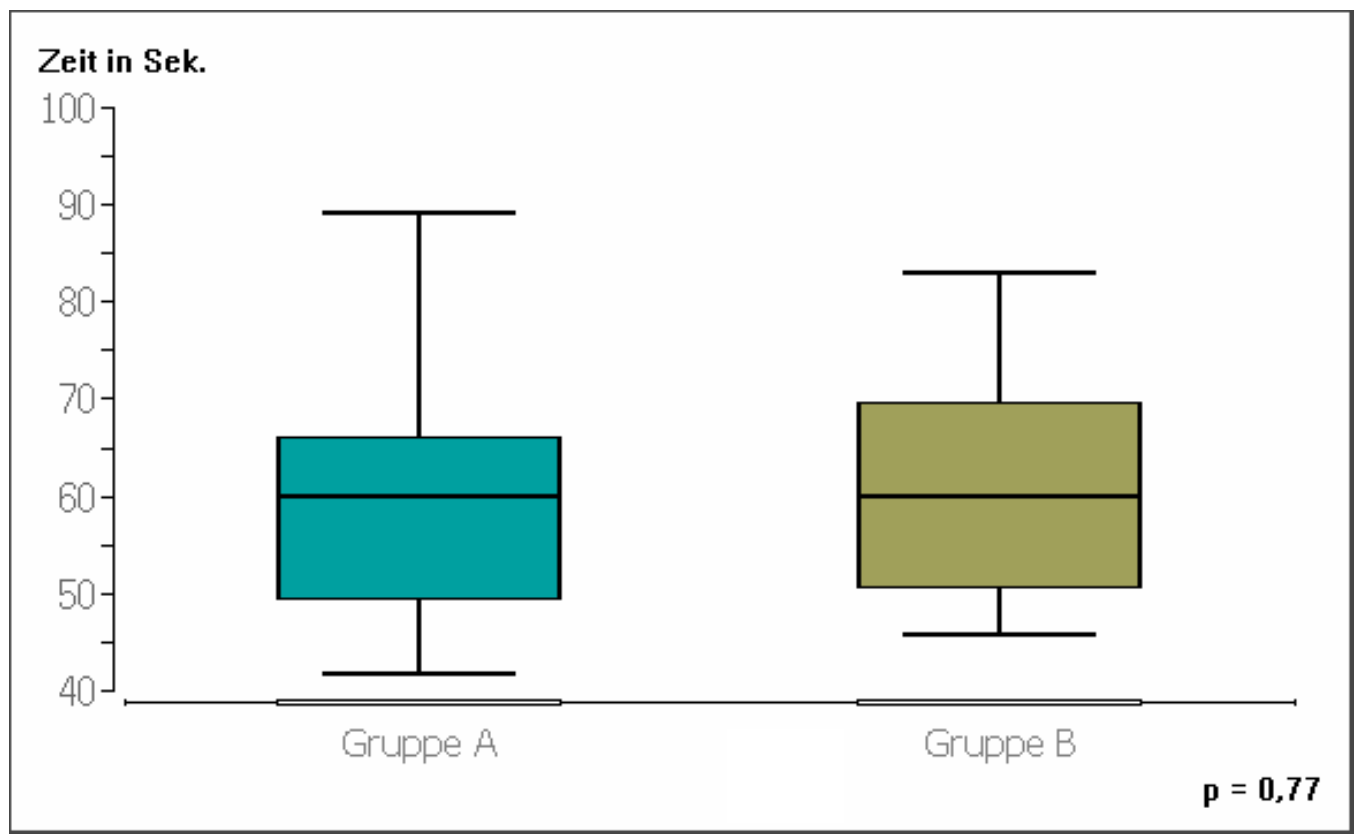




\subsubsection{Aufgabe 2}

"Lokalisieren Sie die Pathologie in der vorliegenden Angiografie".

Alle Probanden (100\%) konnten eine hochgradige Stenose der rechten A. femoralis superficialis (Anh.6) ohne signifikante Zeitunterschiede $(p=0,66)$ feststellen (Abb.12).

Abbildung 12 Bearbeitungszeit Aufgabe 2

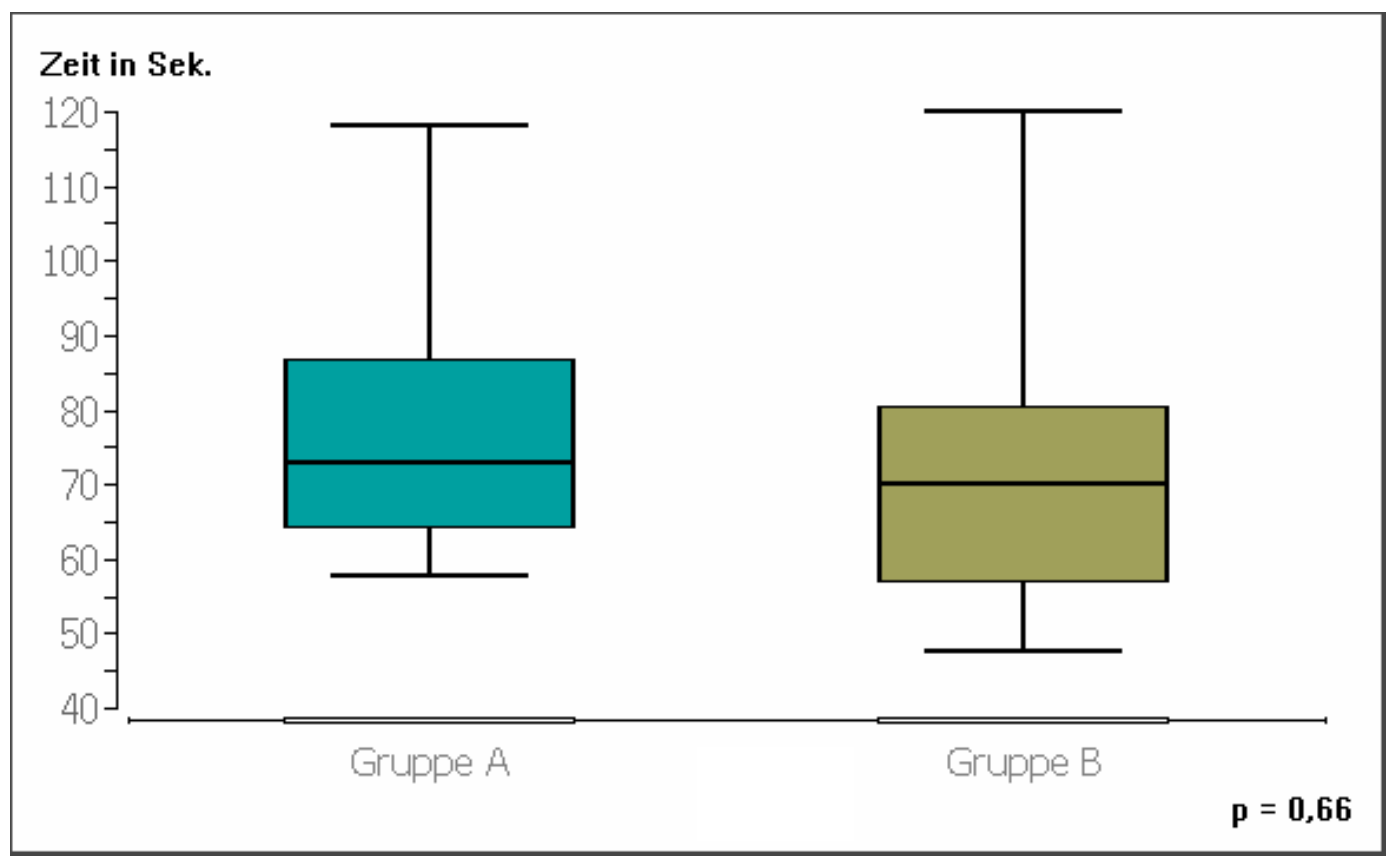


Nach Abzug des Zeitverlustes durch Abstürze der Glass App konnte die Stenose durch Teilnehmer der Gruppe B signifikant schneller $(p<0,01)$ diagnostiziert werden (․ㅣb.13).

Abbildung 13 Bearbeitungszeit Aufgabe 2 abzüglich der App-Neustarts

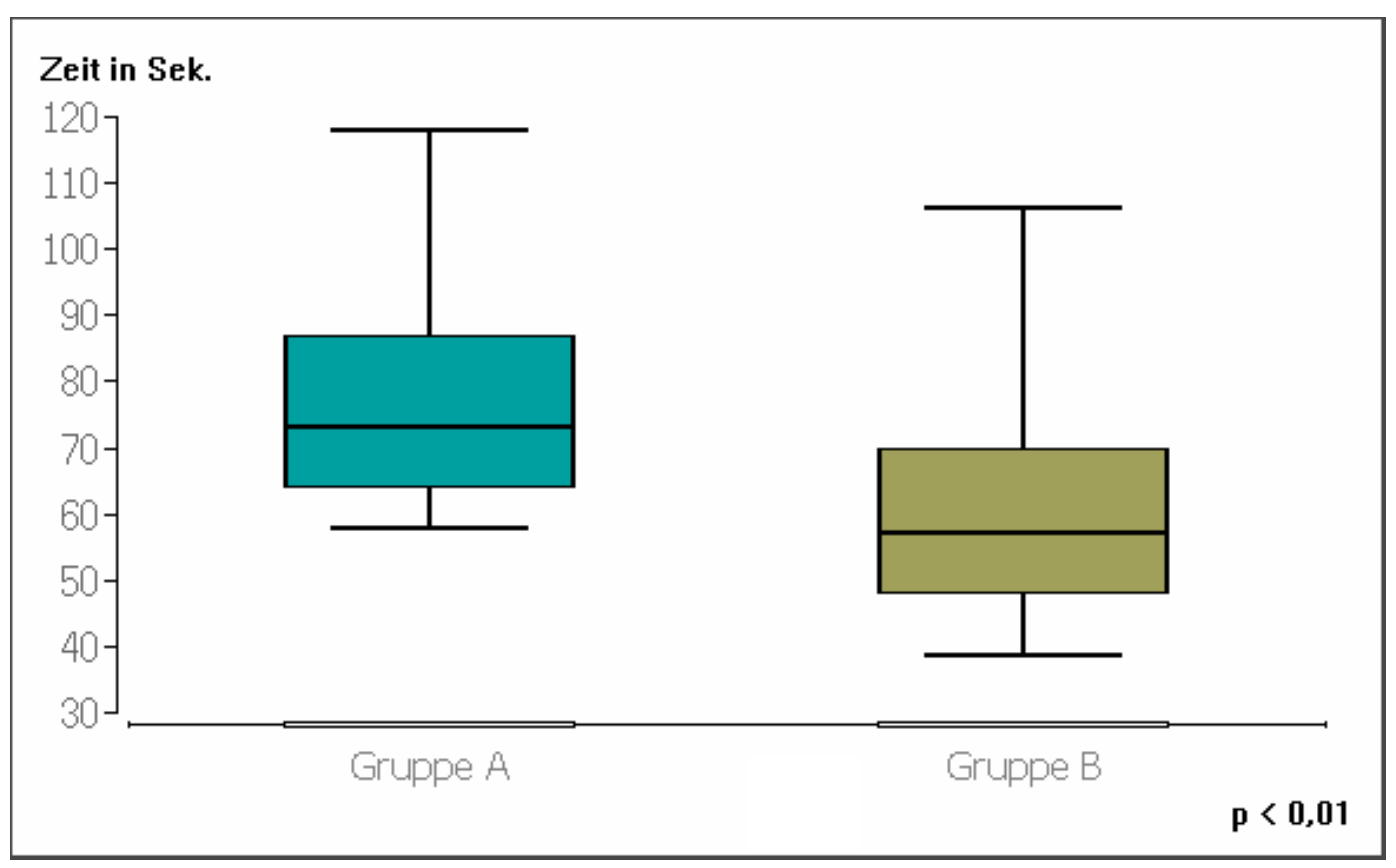




\subsubsection{Aufgabe 3}

"Beurteilen Sie die vorliegenden Laborwerte in Hinsicht auf relevante Parameter".

Die von der Norm abweichenden Laborwerte wurden durch alle Probanden (100\%) korrekt erkannt und wiedergegeben (Anh.7). Unterschiede zwischen den Gruppen hinsichtlich der Diagnosestellung einer Hyperthyreose $(p=0,41)$ und der Relevanz der Nierenwerte im Kontext der simulierten PTA $(p=0,75)$ waren nicht signifikant (․ㅣb.14).

\section{Abbildung 14 Durchführung Aufgabe 3}

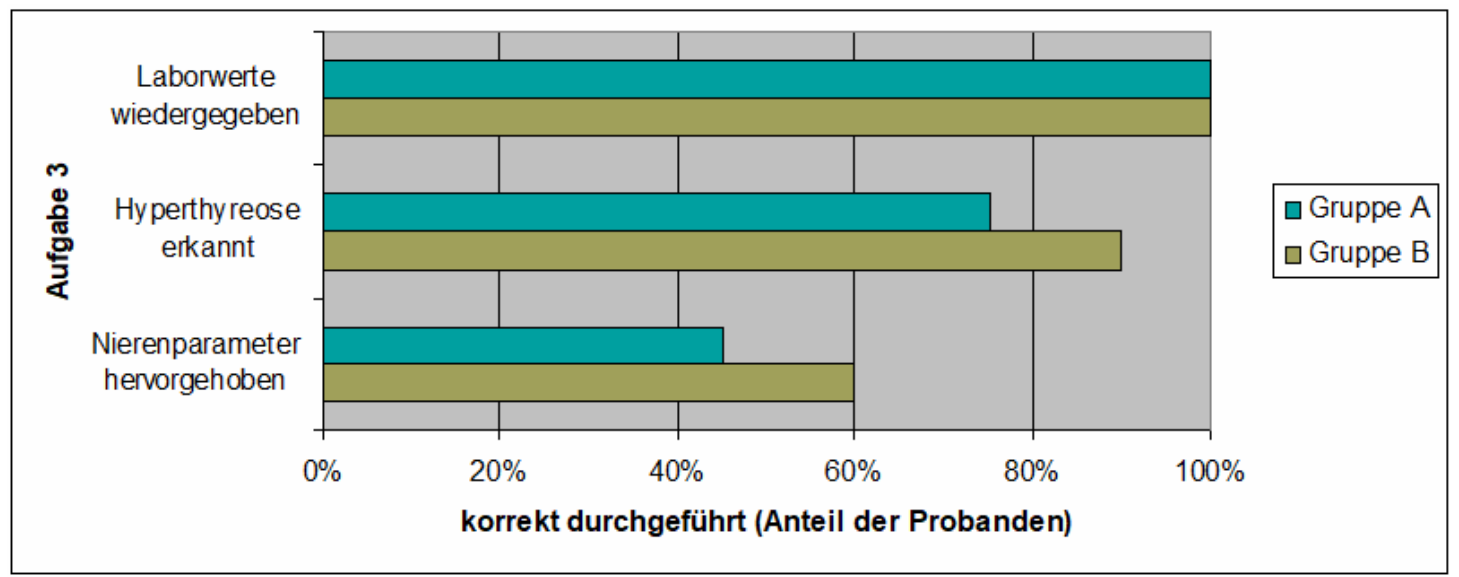


Gruppe B war unter Benutzung der GG sowohl mit $(p=0,01)$, als auch abzüglich des Zeitverlustes durch technische Schwierigkeiten $(p<0,01)$ signifikant schneller in der Durchführung dieser Aufgabe (․ㅣb.15+16).

Abbildung 15 Bearbeitungszeit Aufgabe 3

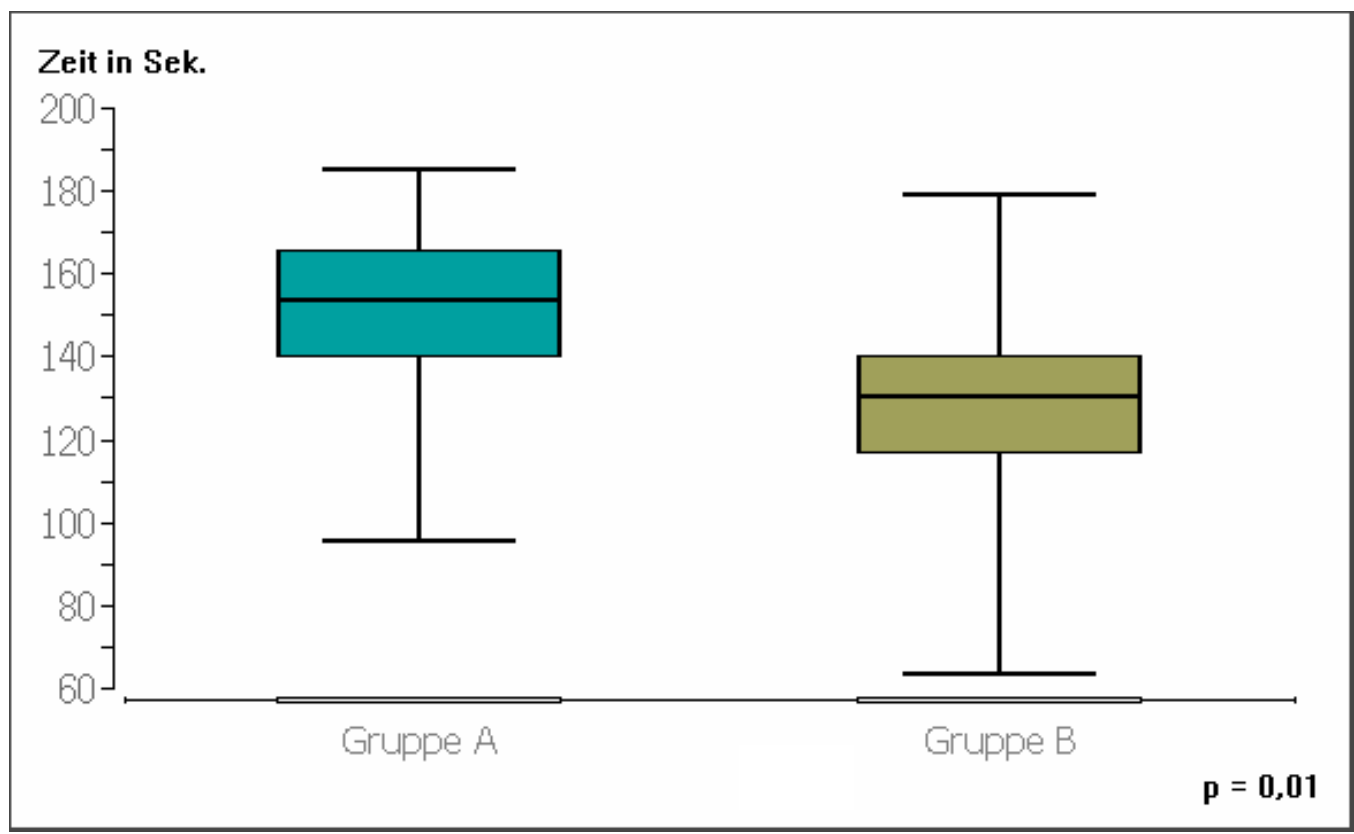

Abbildung 16 Bearbeitungszeit Aufgabe 3 abzüglich der AppNeustarts

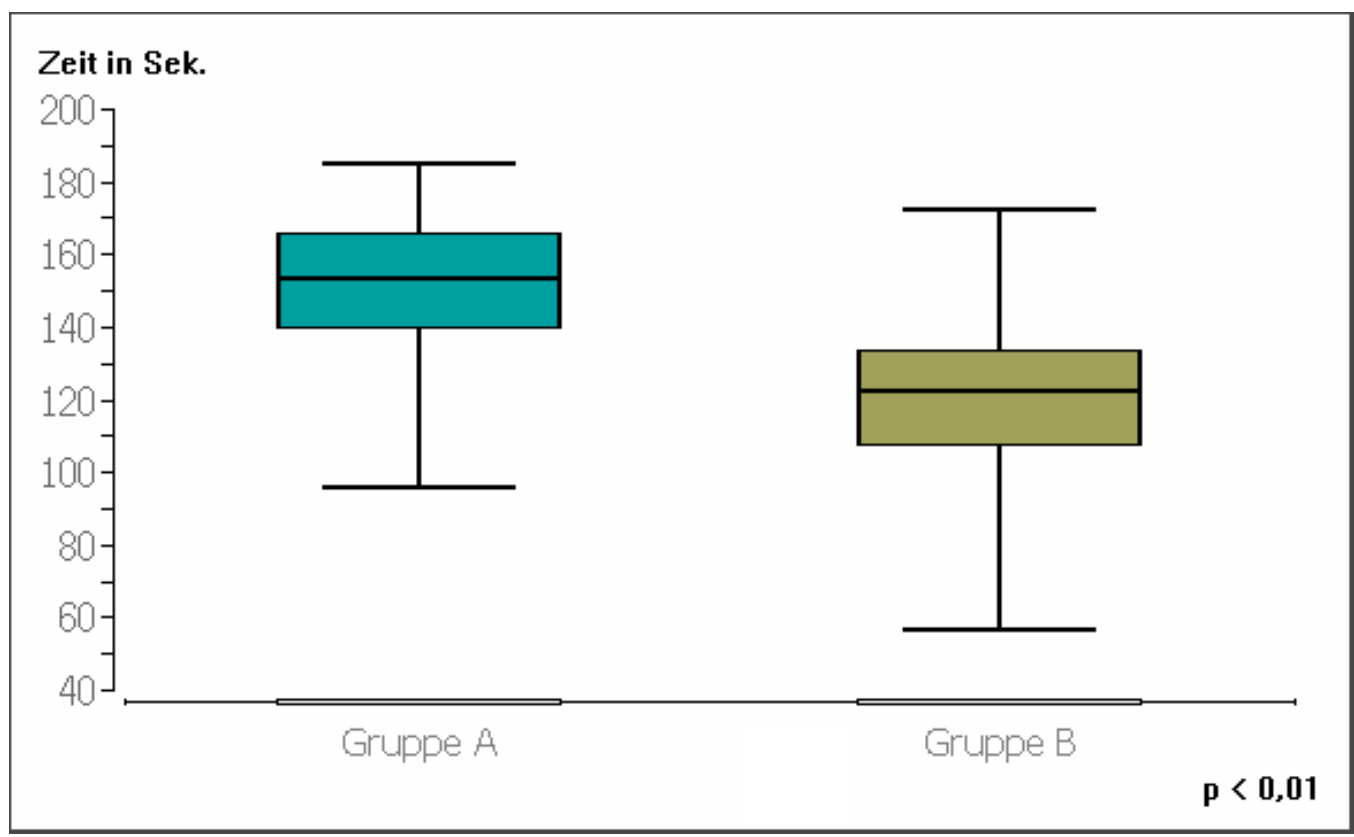




\subsubsection{Aufgabe 4}

"Überprüfen Sie das Monitoring auf kritische Vitalparameter"

Die Kontrolle der Vitalparameter auf pathologische Werte (Anh.8) wurde von beiden Gruppen zeitgleich $(p=0,11)$ und fehlerfrei $(100 \%)$ durchgeführt (Abb.17).

Abbildung 17 Bearbeitungszeit Aufgabe 4

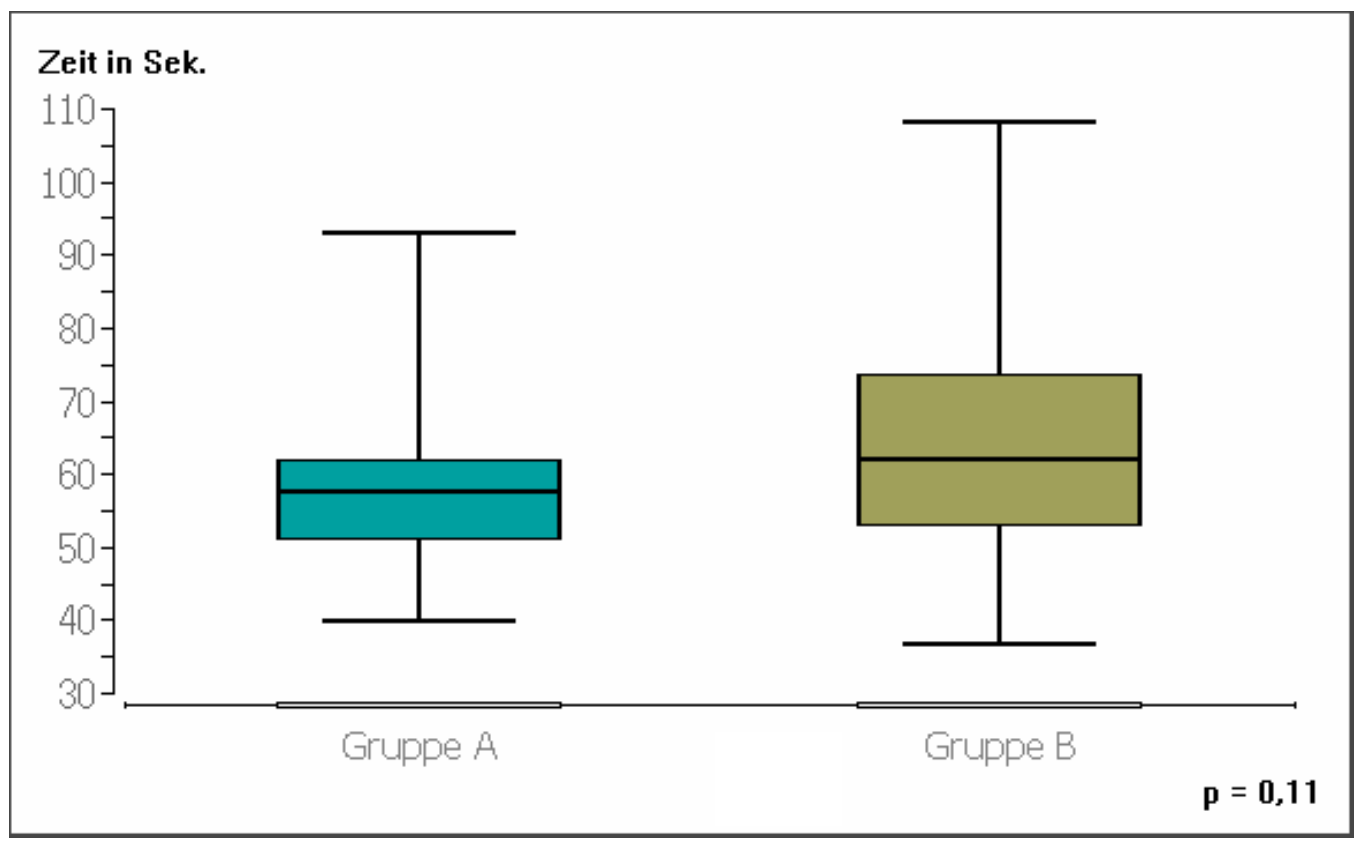


Auch nach Abzug der zusätzlich benötigten Zeit für Neustarts der Custom Glass App in Gruppe B, konnte kein signifikanter Unterschied $(p=0,58)$ zwischen beiden Gruppen festgestellt werden (․ㅣb.18).

Abbildung 18 Bearbeitungszeit Aufgabe 4 abzüglich der App-Neustarts

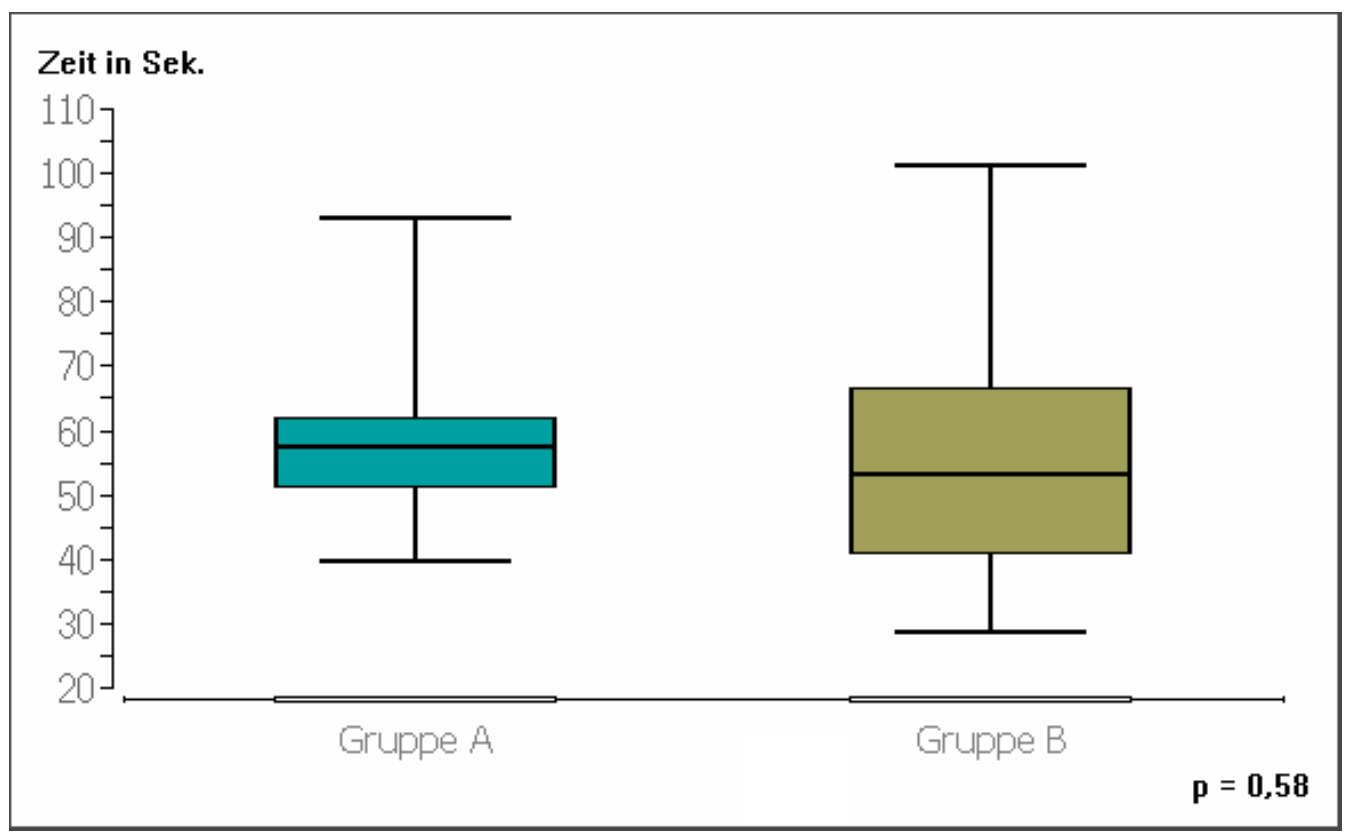




\subsubsection{Aufgabe 5}

"Kontrollieren und bewerten Sie die postinterventionelle Fluoroskopie"

Die fluoroskopische Überprüfung der korrekten Stentplatzierung (Anh.9) wurde von beiden Gruppen erfolgreich durchgeführt (100\%). Eine im Rahmen des Studiendesigns vorgetäuschte Verwechslung des simulierten Patienten, mit ähnlichem fluoroskopischen Befund, ist hierbei ohne signifikanten Unterschied $(p=0,71)$ von weniger als einem Drittel der Probanden in Gruppe A und Gruppe B bemerkt worden (Abb.19).

\section{Abbildung 19 Durchführung Aufgabe 5}

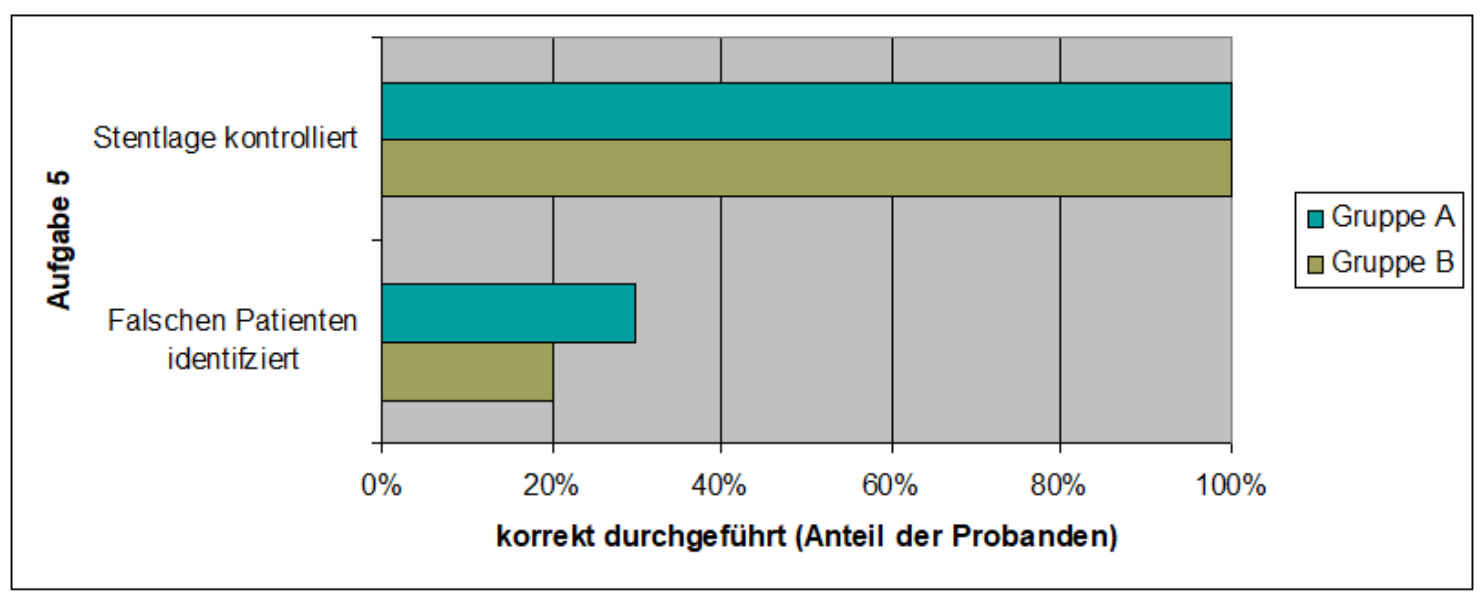


Die Bearbeitungszeit beider Gruppen unterschied sich jedoch signifikant $(p<0,01)$ zu Gunsten des klassischen Monitorings in Gruppe A (ㅎbb.20).

Gruppe B war unter Benutzung von GG, auch abzüglich der zusätzlich benötigten Zeit aufgrund technischer Schwierigkeiten, signifikant langsamer $(p<0,01)$ bei der Bearbeitung dieser Aufgabe ( $\underline{\mathbf{A b b} .21)}$.

Abbildung 20 Bearbeitungszeit Aufgabe 5

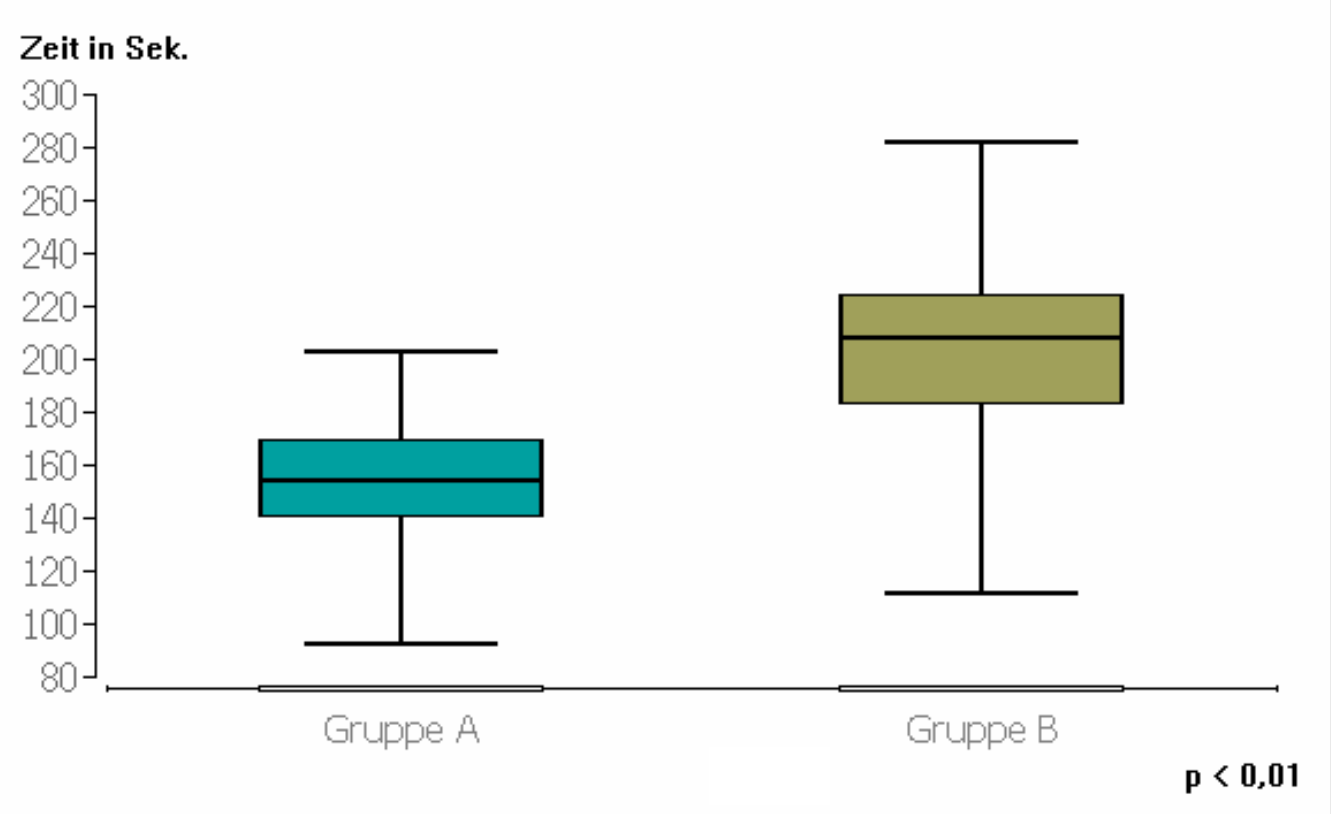

Abbildung 21 Bearbeitungszeit Aufgabe 5 abzüglich der App-Neustarts

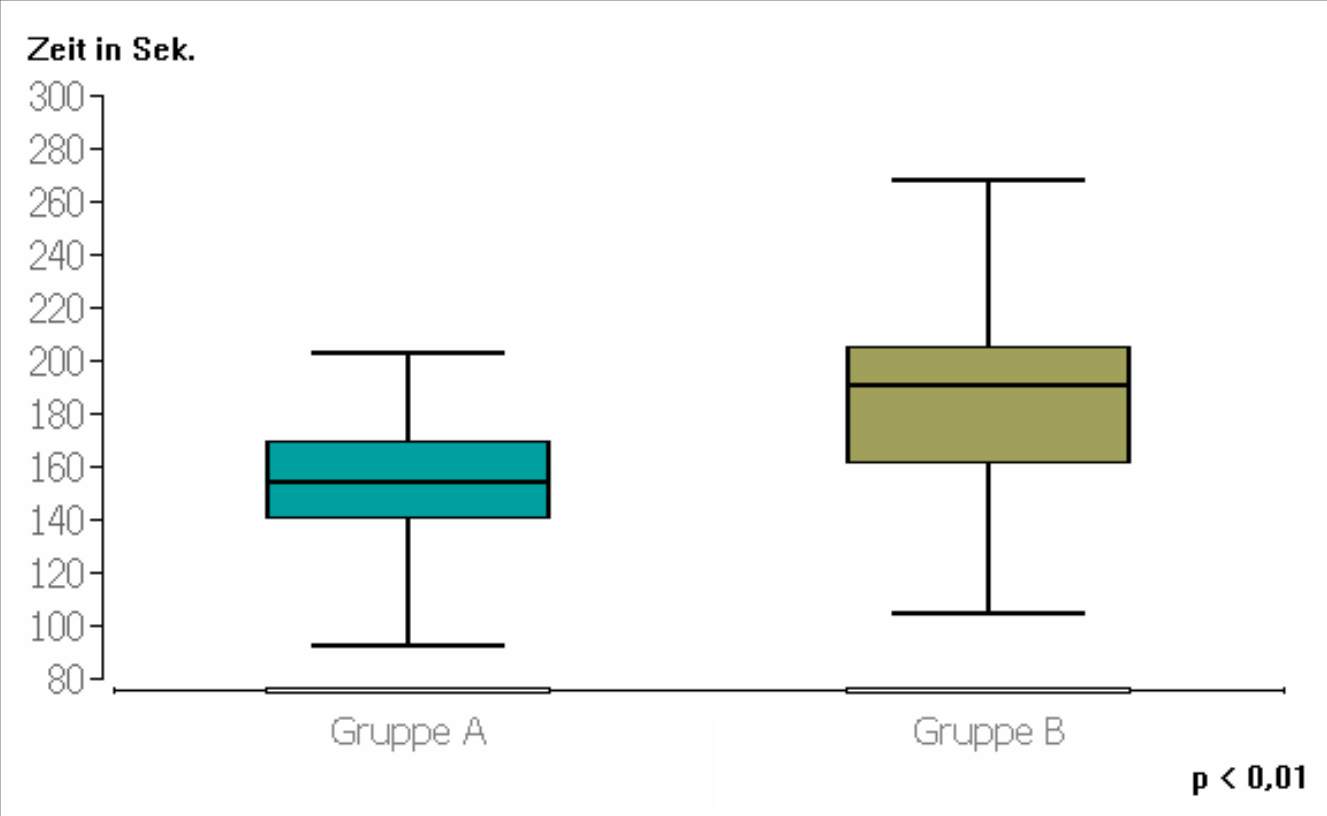




\subsubsection{Aufgabe 6}

"Bewerten Sie die Fotodokumentation der Füße Ihres Patienten"

Die Fotodokumentation der Füße, als Verlaufskontrolle im Rahmen des Fallbeispiels (Anh.10), konnten die Probanden beider Gruppen (100\%) korrekt als eine kontralaterale Gefäßstenosierung in Form eines ischämischen linken Unterschenkels interpretieren.

Gruppe B konnte den Befund hierbei, auch inklusive Zeitverlust durch wiederholtes Abstürzen der Custom Glass App, signifikant schneller bewerten $(p<0,01)$ als Gruppe A (

Abbildung 22 Bearbeitungszeit Aufgabe 6

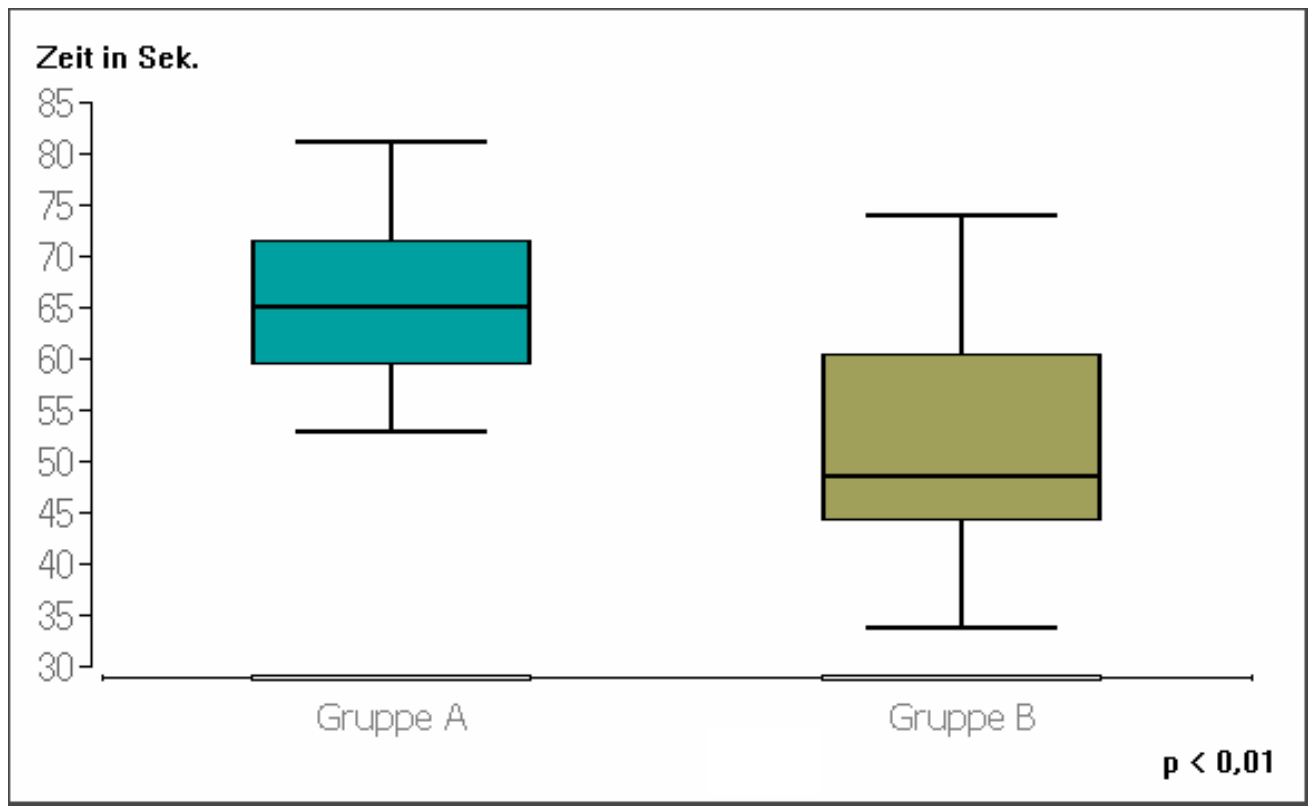


Abbildung 23 Bearbeitungszeit Aufgabe 6 abzüglich der App-Neustarts

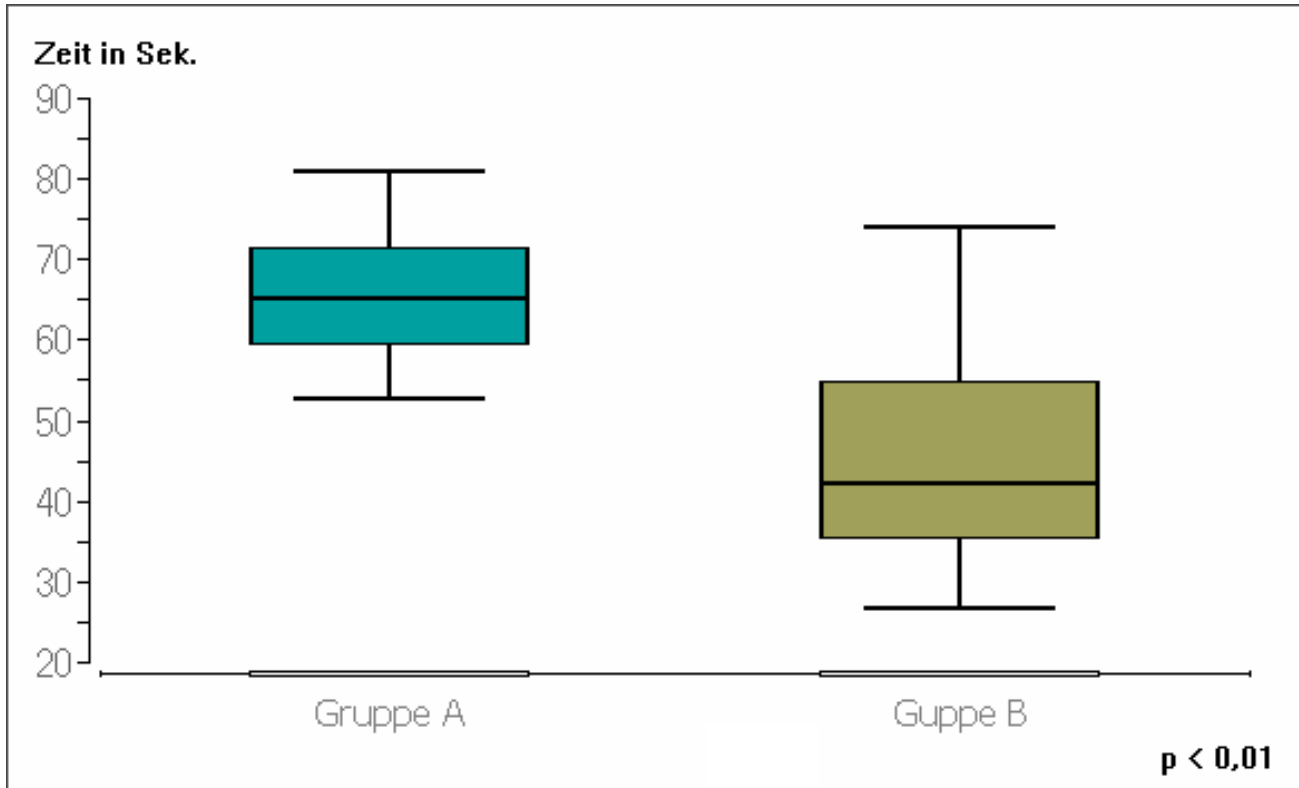




\subsection{Auswertung der persönlichen Fragebögen}

\subsubsection{Zeitmanagement und Kommunikation}

$70 \%$ der Teilnehmer vertraten die Meinung, dass die Eingriffsdauer peripherer endovaskulärer Interventionen durch neue Technologien, wie zum Beispiel oHMD, weiter optimiert werden könnte. Die übrigen $30 \%$ waren sich hierbei jedoch unschlüssig, ob durch weitere Zeiteinsparungen in der Interventionsdauer die Qualität der postoperativen Ergebnisse abnehmen könnte. $60 \%$ waren der Ansicht, dass die direkte, persönliche Interaktion zwischen den Mitarbeitern eines Katheterlabors eine wertvolle Ressource ist und Verzögerungen im Interventionsablauf durch mangelnde Kommunikation auftreten könnten. Beide Gruppen bewerteten die personellen Interaktionen im Katheterlabor weder als störend in Bezug auf die Qualität des Eingriffs, noch als einen relevanten Stressfaktor hinsichtlich des Ablaufs der Interventionen.

$65 \%$ aller Teilnehmer zogen es vor, unabhängig und selbstständig arbeiten zu können, während 92,5\% es als hilfreich empfanden, zu jeder Zeit des Eingriffs in Interaktion mit einer OP-Schwester treten zu können.

Hierbei kann es sich zum Beispiel um die gemeinsame Abstimmung geplanter Interventionsschritte oder den gemeinsamen Austausch von Fachwissen handeln, um die Interventionsbedingungen für das autonome Vorgehen des Operateurs zu optimieren.

\subsubsection{Informationsfluss und Digitalisierung im klinischen Alltag}

Insgesamt $70 \%$ aller Probanden bewerteten den Informationsgewinn durch die Nutzung digitaler Medien gegenüber gleichwertigem Material in analoger Darstellung als eher $(52,5 \%)$ bis eindeutig höher $(17,5 \%)$. Die restlichen $30 \%$ der Probanden betrachteten den Informationsgewinn als mindestens gleichwertig (15\%) oder sogar eher $(12,5 \%)$ bis eindeutig niedriger $(2,5 \%)$ (Abb.24). Hierzu zählen alle Texte, Abbildungen, Video- oder Bildsequenzen, die mithilfe eines digitalen Mediums (z.B. PC, Tablet, oHMD) in Echtzeit abrufbar, und vor allem auch modifizier- oder bearbeitbar sind. 
Im Gegensatz dazu stehen analoge Medien im Kontext dieser Studie für alle nicht-digitalen Informationsquellen, wie zum Beispiel Patientenakten, Fotografien in Papierform oder der klassische Röntgenfilm.

$65 \%$ der Probanden bevorzugten demnach den Informationstransfer in digitaler Form, da sie diesen für besser (15\%) halten oder gegenüber der analogen Form eher bevorzugen (50\%). 12,5\% hingegen würden sich für eine analoge Darstellung entscheiden, da sie den digitalen Transfer im Vergleich für schlecht $(5 \%)$ oder zumindest schlechter $(7,5 \%)$ halten. $22,5 \%$ machten keinen Unterschied bei der Wahl eines analogen oder digitalen Informationsflusses (Abb.25). 95\% aller Probanden betrachteten die Digitalisierung im klinischen Alltag als sinnvoll und nützlich.

In diesem Sinn vertrat die Mehrheit der Probanden (82,5\%) die Ansicht, dass der Einsatz neuer digitaler Technologien, wie beispielsweise GG, zu Zeiteinsparungen bei peripheren endovaskulären Katheterinterventionen führen könnte. Alle Teilnehmer (100\%) vertraten den Standpunkt, dass das Prinzip der AR sinnvoll in den Arbeitsablauf im Katheterlabor integriert werden könnte.

Abbildung 24 Informationsgewinn aus digitalen gegenüber analogen Medien

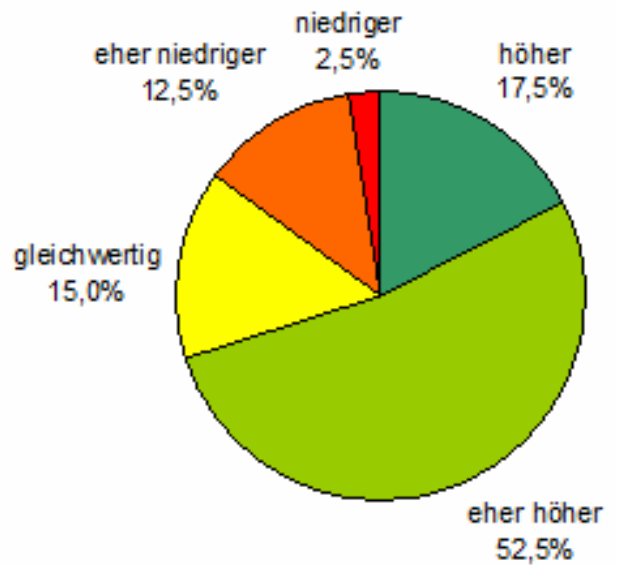

Abbildung 25 Informationstransfer in digitaler gegenüber analoger Form

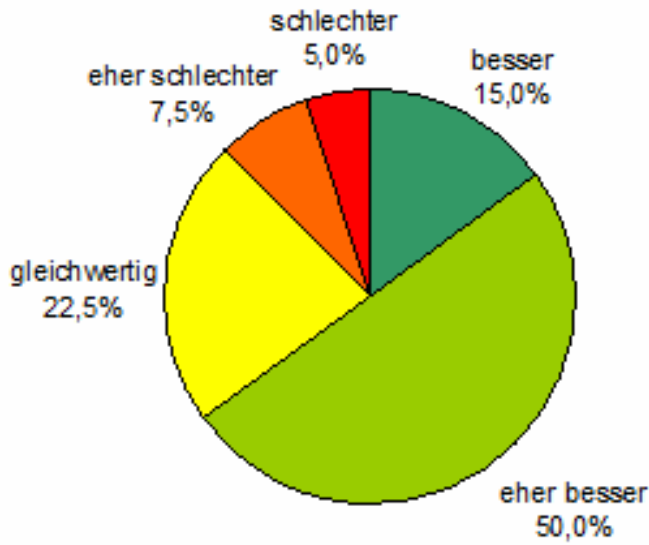




\subsubsection{Handhabung von Google Glass ${ }^{\circledR}$}

Die Probanden der Gruppe B empfanden die Handhabung von GG überwiegend als einfach bis sehr einfach (85\%). Der Tragekomfort des Gerätes wurde von 16 der 20 Probanden (80\%) als gut bis ausgezeichnet bewertet. $65 \%$ fühlten sich durch das Tragen von GG in ihrem Sichtfeld eher $(30 \%)$ bis eindeutig (35\%) ergänzt. 30\% hielten dabei den Einfluss des Geräts auf das Sichtfeld für weder besonders einschränkend noch vorteilhaft. Nur ein Proband (5\%) sah die Benutzung von GG im Hinblick auf das eigene Sichtfeld als Einschränkung (시‥26).

Die Bildqualität der abrufbaren Grafiken und das Prinzip der Spracherkennung wurden von jeweils ungefähr einem Drittel der getesteten Personen als gut, durchschnittlich und als schlecht bewertet $(\underline{A b b .27+28})$. 95\% aller Probanden der Gruppe B konnten sich die Verwendung von GG während peripheren endovaskulären Eingriffen in Zukunft gut vorstellen.

Abbildung 26 Einfluss auf das Sichtfeld bei der Benutzung von Google Glass $₫$

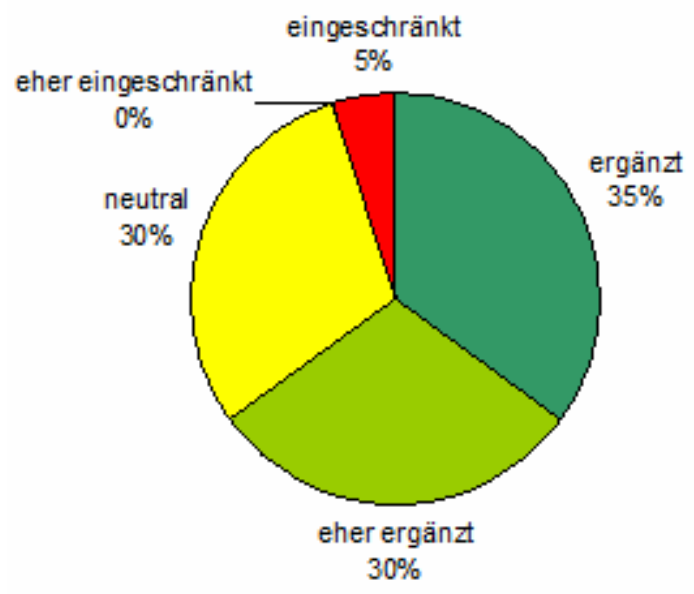


Abbildung 27 Bildqualität der Grafiken bei der Benutzung von Google Glass $®$

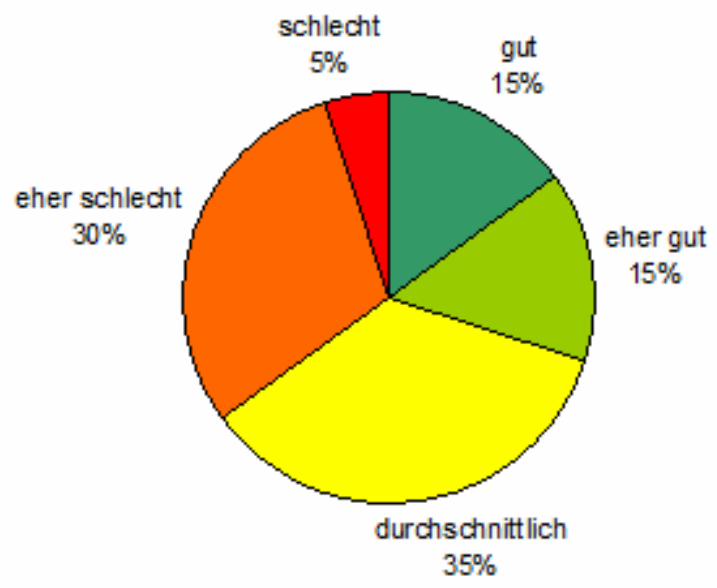

Abbildung 28 Sprachsteuerung bei der Benutzung von Google Glass ${ }^{\circledR}$

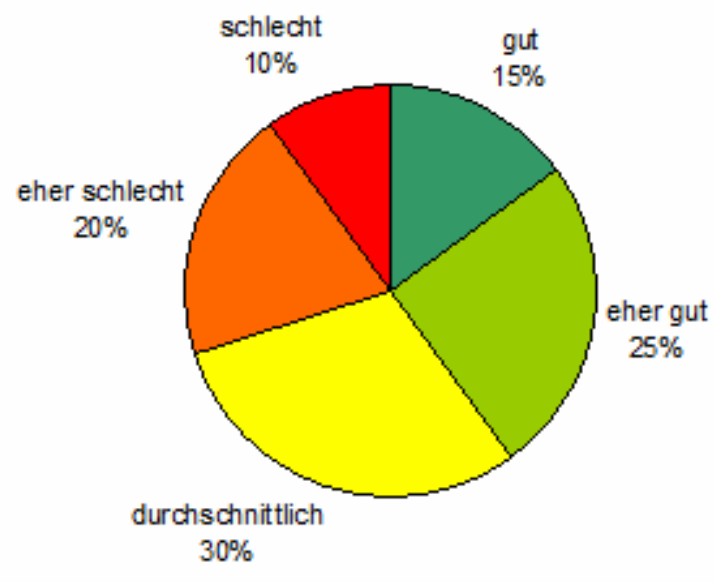

\subsection{Zusammenfassung der Ergebnisse}

Hinsichtlich der Fragestellung dieser Studie lassen sich in Zusammenschau der Ergebnisse folgende Aussagen treffen:

a) GG kann für den Operateur bei einer peripheren endovaskulären Katheterintervention als bildgebendes Medium hilfreich sein, um sich einfache visuelle Informationen schnell und autonom zugänglich zu machen.

b) Die Custom Glass App ist, aufgrund von technischen Schwierigkeiten mit der Spracherkennungsfunktion und Bildqualität, noch nicht zuverlässig während einer peripheren endovaskulären Katheterintervention einsetzbar.

c) Der Arbeitsablauf einer peripheren endovaskulären Katheterintervention kann durch die kombinierte Nutzung der Custom Glass App und GG verbessert werden, jedoch sollten die aufgedeckten technischen Probleme zur Optimierung der Anwendbarkeit im Katheterlabor noch überwunden werden. 


\section{DISKUSSION}

Da GG eine der ersten kompakten oHMD ist, wurde sie auch in einigen anderen Studien diverser medizinischer Disziplinen auf ihre verschiedenen Funktionen hin getestet. Hierzu zählen unter anderem die drahtlose Überwachung der Vitalfunktionen in der Anästhesiologie (9), die Aufzeichnung von chirurgischen Eingriffen in der Augenheilkunde (10), das Echtzeit-Streaming von Tumorresektionen in der Neurochirurgie (11), die EKG-Befundung in der Kardiologie (12), die fotografische Dokumentation in der Gerichtsmedizin (13), die dermatologische Telekonsultation (14) und die visuelle Unterstützung bei klinischen Simulationen (15), um nur einige Beispiele zu nennen.

85\% aller an unserer Studie teilnehmenden Probanden waren der Meinung, dass der Informationsgewinn digitaler Medien größer oder zumindest gleichwertig der analogen Form ist. $65 \%$ der Befragten bevorzugten aufgrund dessen den Einsatz digitaler Medien. Unsere Studie zeigte eine durchweg positive Einstellung (92,5\%) gegenüber der Digitalisierung im klinischen Altag. In diesem Zusammenhang waren alle Probanden (100\%) der Ansicht, dass das Prinzip der AR sinnvoll in periphere endovaskuläre Verfahren integriert werden kann.

Diese allgemein positive Haltung gegenüber dem Einsatz von $A R$ in chirurgischen Fachdisziplinen wurde auch 2017 durch Vávra et al. (16) in einem Review von insgesamt 102 Studien bestätigt. Über den Einsatz von AR im Kontext endovaskulärer Interventionen wurde unter anderem 2018 von Grinshpoon, Sadri et al. (17) berichtet, welche die interaktive Navigation durch 3D-Projektionen anatomischer Strukturen, ausschließlich durch Kopfbewegungen und Sprachsteuerung mithilfe eines oHMD, ermöglichten.

Lu, Wang et al. (18) beschrieben in einem Case Report von 2019 die erfolgreiche, interventionelle Revaskularisierung eines Unterschenkels bei Totalverschluss der A. poplitea durch ein AR-assistiertes Navigationssystem per oHMD. 


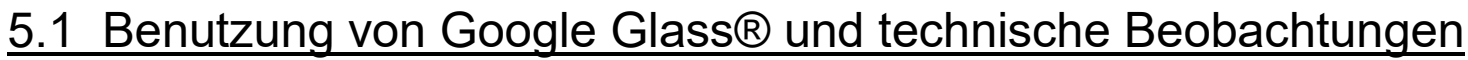

Während sich die meisten Studien mit GG auf die Netzwerkfähigkeit oder die Foto- und Videofunktionalität des Gerätes beziehen, konzentrierten wir uns im Rahmen unserer Studie auf die Fähigkeit der Spracherkennung und die sprachgetriggerte Bereitstellung grafischer Informationen. Die grundlegende Idee war es, das Gerät als intraoperatives Medium in der Gefäßchirurgie für den Operateur nutzbar zu machen, um visuelle Informationen während der PTA eines peripheren Gefäßverschlusses autonom abzurufen.

\subsubsection{Allgemeine Handhabung und Tragekomfort}

Die Ergebnisse der Gruppe B (Experimentalgruppe) unterstreichen die einfache und intuitive Handhabung von GG nach einer kurzen Eingewöhnungsphase, wie bereits von Chaballout et al. (15) und Yoon et al. (19) berichtet wurde.

Unsere Studie bestätigte einen hohen Tragekomfort aufgrund des leichten und schlanken Designs des Geräts, wie auch durch Albrecht et al. (13) und DrakeBrockman et al. (20) beschrieben.

Die individuelle Einstellung des GG-Projektors (Prisma) an die Gesichtsform des Benutzers spielte bei der Handhabung im Rahmen unserer Studie eine tragende Rolle, da die Projektion im seitlichen Sichtfeld verschwommen erschien, wenn das Gerät zuvor nicht akkurat an den Benutzer angepasst wurde. Ein Eyetracking-Modul, also ein System zur Ausrichtung des Prismas anhand der Blickrichtung des Benutzers, könnte eine technische Lösung sein, um die individuelle Anpassung von GG zu verbessern oder diese sogar zu automatisieren.

\subsubsection{Betriebs- und Akkulaufzeit}

Aungst et al. (21) berichteten, bei einer Betriebszeit von GG zwischen 30 Minuten und vier Stunden, von einer erhöhten Erwärmung des Geräts in Abhängigkeit von den Betriebsanforderungen an GG. Aufgrund der Tatsache, dass die visuelle Information im Rahmen unserer Studie aus einzelnen Bildern statt aus Videos bestand, trat keine unangenehme Erwärmung von GG auf, wie zuvor von Diaz et al. (11), Chaballout et al. (15) oder Mishra et al. (22) berichtet. 
Wir konnten die Schwierigkeiten bezüglich der Akkulaufzeit, wie sie von Chang et al. (23), Münsterer et al. (24) und Borgmann et al. (25) kritisiert wurden, dementsprechend ebenfalls nicht bestätigen. Dies ist am ehesten auf die vergleichsweise kurze Betriebszeit von GG in unserem simulierten Eingriff (zwischen 11 und 16 Minuten) oder auf andere technische Ursachen des Geräts zurückzuführen, die seitens des Herstellers Google möglicherweise noch nicht aufgedeckt wurden.

Auch wenn die Betriebszeit der GG während unserer Simulation kürzer war als die Interventionszeit einer realen PTA, schließen wir daraus, dass diese technischen Details, bei der Verwendung von GG während einer PTA der A. femoralis superficialis im Sinne unserer Studie, keine relevanten Auswirkungen haben würden.

\subsubsection{Custom Glass App und Sprachsteuerung}

Während unserer Testungen konnten wir Herausforderungen bezüglich der Spracherkennungsfunktion bei der Benutzung der Custom Glass App feststellen. Da die App das Mikrofon von GG, und damit einhergehend die Spracherkennungsfunktion, permanent aktiviert lässt, um jederzeit und spontan auf die Sprachbefehle des Operateurs zu reagieren, konnte GG regelmäßig nicht sicher zwischen der Stimme des Benutzers und anderen Stimmen oder Geräuschen in der näheren Umgebung unterscheiden. Dies hatte zur Folge, dass kurze Sprachbefehle aufgrund der Störgeräusche teilweise nicht präzise erkannt und falsches Bildmaterial angezeigt wurde. Die App wurde auffallend häufig mit akustischen Signalen - teils korrekte Sprachbefehle und teils Störsignale - überlastet, was zu plötzlichen Software-Abstürzen führte. Diese erforderten insgesamt zwischen 4 und 18 Neustarts der App pro getestetem Probanden der Gruppe B, was einem Zeitverlust von 28 bis 126 Sekunden pro simuliertem Eingriff entspricht. 
In diesem Zusammenhang hat sich die Sprachsteuerungsfunktion der Custom Glass App als verbesserungswürdig herausgestellt und stellt zugleich eine Herausforderung in Bezug auf die mögliche Geräuschkulisse in einem Katheterlabor dar.

\subsubsection{Bildqualität und Beeinflussung des Sichtfeldes}

Die Bildqualität des GG-Projektors (Prisma) wurde bereits von Albrecht et al. (13), Chang et al. (23) oder Münsterer et al. (24) kritisiert. In unserer Studie wurden Texte in Abhängigkeit der Schriftgröße sowie Details komplexer Grafiken vereinzelt unpräzise abgebildet. Jedoch konnte durch diese Unschärfe keine Einschränkung bei der Durchführung der PTA beziehungsweise bei der Bearbeitung der gestellten Aufgaben während des simulierten Fallbeispiels beobachtet werden.

Hervorzuheben ist hierbei, dass es verpflichtende Mindestanforderungen an Bildwiedergabegeräte zur radiologischen Befundung gibt, welche im Rahmen von Abnahme- und Konstanzprüfungen der Geräte kontrolliert werden. Eine Mindestanforderung zur Befundung von Durchleuchtungen, wie sie im Katheterlabor üblich sind, ist zum Beispiel eine Bildauflösung von mindestens 1024 x 1024 Pixel (26). Damit kann GG mit einer Projektor-Auflösung von 640 x 360 Pixel (Tab.2) bereits formal nicht zur Befundung im Katheterlabor zugelassen werden. In unserer Studie wurde GG dementsprechend zur Betrachtung zusätzlicher visueller Informationen neben der klassischen Anordnung von zwei Befundungsmonitoren eingesetzt.

Wir konnten bestätigen, dass sich die Kontrastqualität in Abhängigkeit von den Lichtbedingungen des Hintergrunds veränderte, wie auch Münsterer et al. (24) beschrieben. Unserer Ansicht nach sollte GG durch einen integrierten Umgebungslichtsensor verbessert werden, der den Kontrast und die Helligkeit automatisch an die Umgebung anpasst, wie es zum Beispiel bereits bei modernen Smartphones der Fall ist. 
Um einer möglichen grafischen Unschärfe entgegenzuwirken, wäre eine, wie von Albrecht et al. (13) oder Münsterer et al. (24) vorgeschlagene, ZoomFunktion denkbar. Texte oder spezifische Details einer Grafik könnten so auf das benötigte Maß vergrößert werden, um diese für den Benutzer von GG klar abzubilden. Sprachbefehle oder bestimmte Kopf- oder Augenbewegungsmuster wären technisch denkbar, um einen digitalen Zoom für die Vergrößerung bestimmter Details zu aktivieren.

Insgesamt empfanden 65\% unserer Probanden in Gruppe B die Benutzung von GG und den damit einhergehenden, zusätzlichen Informationsgewinn als positive Ergänzung ihres natürlichen Sichtfeldes. Nur ein Proband (5\%) beurteilte die Verwendung von GG, aufgrund der genannten Defizite der Bildqualität, subjektiv als Einschränkung ( $\underline{\text { Abb.26). }}$.

Die Version von GG, die wir für unsere Studie verwendeten, war für Brillenträger mit eingeschränkter Sehfähigkeit, wie von Vorraber et al. (27) dokumentiert, immer noch ungeeignet. Probanden mit Sehstörungen konnten die angezeigten Texte und relevanten Details durch GG dementsprechend kaum erkennen und wurden daher nachträglich von unserer Studie ausgeschlossen.

Dieses Problem wurde jedoch zwischenzeitlich durch das Unternehmen Google erkannt und gelöst, indem GG nun auch mit Brillengestellen erhältlich ist, die mit Korrekturlinsen ausgestattet werden können (28).

\section{$\underline{5.2 \text { Bildgebung im Katheterlabor }}$}

Die Basis eines jeden interventionellen endovaskulären Verfahrens ist eine qualitativ hochwertige Bildgebung sowie eine effektive Beobachtung des Eingriffs über mehrere Monitore. Dementsprechend können in Katheterlaboren, je nach Komplexität der Prozedur, bis zu sechs oder mehr verschiedene Monitore zur Informationsübermittlung gefunden werden. In modernen AngioSuiten kann die gleiche Informationsmenge auch auf einem einzigen Breitbildmonitor angezeigt werden, der sich auf die individuellen Anforderungen des behandelnden Operateurs anpassen lässt. 
In diesem Zusammenhang ist es fraglich, wie viele Informationen ein Operateur im Rahmen eines interventionellen Eingriffs visuell aufnehmen und verarbeiten kann, ohne gleichzeitig von der eigentlichen Intervention abgelenkt zu werden.

\subsubsection{Visuelle Wahrnehmung und Verarbeitung}

Halford, Baker et al. (29) konnten unabhängig von der Thematik dieser Studie zeigen, dass maximal vier verschiedene grafisch dargestellte Variablen gleichzeitig durch das menschliche Gehirn verarbeitet und in einen gemeinsamen Kontext gebracht werden können. N. Cowan (30) beschrieb zuvor ebenfalls eine Speicherkapazitätsgrenze des Kurzzeitgedächtnisses von vier Variablen bei der Verarbeitung visueller Informationen. Auch wenn die genannten Beobachtungen unter kontrollierten Bedingungen und mit einfachen grafischen Darstellungen, wie beispielsweise Diagrammen oder Zahlenreihen, gemacht wurden, so ist es naheliegend, dass der Informationsgehalt von bereits einem Monitor im Katheterlabor, wie einer Angiografie, wesentlich komplexer und die Verarbeitung durch den Operateur damit deutlich schwerer ist. Mehr als vier Monitore - oder auch Fenster in einem Breitbildmonitor - überschreiten demnach wahrscheinlich die Grenze der Verarbeitungskapazität des Operateurs im Katheterlabor und könnten eine mögliche Ablenkung darstellen. Dies sollte als Gegenstand weiterer Studien ausführlich untersucht werden.

\subsubsection{Ablenkung durch visuelle Eindrücke}

Die visuell-motorische Verbindung zwischen Augen und Händen, die sogenannte Auge-Hand-Koordination, ist ein Grundprinzip interventioneller Verfahren. Hemond et al. (31) bewiesen, dass motorische Fähigkeiten durch visuelle Eindrücke, abhängig von der Aktivierung ähnlicher oder verschiedener Prozesse in unserem Gehirn, entweder beeinträchtigt oder verbessert werden können. Die Erfahrungen im Rahmen unserer Studie bestätigten die Ergebnisse von Hemond et al. nochmals. Die visuellen Eindrücke des Live-AngiografieMonitorings und die motorischen Fähigkeiten der Probanden bei der Benutzung von Drähten und Kathetern ergänzten sich während der simulierten PTA, da es sich hierbei um eine visuelle Bestätigung der manuellen Fertigkeiten in Echtzeit handelte. 
Die haptischen und optischen Informationen ließen sich somit effektiv integrieren und begünstigten so einen flüssigen Arbeitsablauf bei der Durchführung der PTA.

Im Gegensatz dazu wurde die simulierte Intervention von nahezu allen Probanden deutlich beeinträchtigt oder sogar kurzzeitig unterbrochen, um die parallel gestellten Aufgaben (Tab.1) anhand der zusätzlichen visuellen Informationen über einen weiteren Monitor (Abb.2) bearbeiten zu können. In diesen Fällen hatte die visuelle Information keinen unmittelbaren Zusammenhang mit der Benutzung der Drähte oder Katheter während der Intervention, sodass die motorischen Fähigkeiten durch die zusätzlichen visuellen Eindrücke gestört wurden.

In Zusammenschau dieser Beobachtungen gehen wir davon aus, dass die motorischen Fähigkeiten des Operateurs, abhängig von Inhalt und Kontext der visuellen Informationen, bereits durch wenige zusätzliche Monitore beeinträchtigt werden können. Aus unserer Sicht könnte demnach eine Kombination von nur zwei Monitoren, bestehend aus einem Live-AngiografieMonitor zur Echtzeitüberwachung der Intervention und einem Referenz-Monitor zur Bildspeicherung, durch GG ergänzt werden, um einen möglichst effektiven Informationsfluss zu gewährleisten.

Dadurch bestünden insgesamt nur drei bildgebende Medien, sodass nach Halford, Baker et al. (29) und N. Cowan (30) eine mögliche Kapazitätsgrenze der Informationsverarbeitung des menschlichen Gehirns nicht überschritten wird und sich die Wahrscheinlichkeit etwaiger Ablenkungen reduziert.

Durch die Anpassungsfähigkeit eines oHMD an den Informationsbedarf des Operateurs wäre dieser in der Lage, sich zusätzlich benötigte Informationen selbstständig per Sprachbefehl an den relevanten und im Interventionsablauf günstigsten Zeitpunkten abzurufen. Hierdurch würden störende visuelle Einflüsse auf die motorischen Fähigkeiten des Operateurs durch weitere Monitore in Anlehnung an Hemond et al. (31) reduziert werden. 


\subsection{Analyse der Aufgabenauswertung}

In Hinblick auf die Leistungen beider Gruppen während der Simulation, konnten Vor- und Nachteile der bildgebenden Verfahren festgestellt werden.

So zeigte sich, unabhängig von technischen Schwierigkeiten oder Softwareproblemen, ein signifikanter zeitlicher Vorteil von GG gegenüber dem klassischen Monitoring bei der Kontrolle von tabellarisch angeordneten Laborparametern (Tab.1, Aufg.3) und bei der Interpretation einfacher Fotografien (Tab.1, Aufg.6). Daraus lässt sich ableiten, dass der Informationsgehalt klar strukturierter Darstellungen oder übersichtlicher Fotodokumentationen durch die Benutzung von GG schneller verarbeitet werden kann, als im Rahmen der klassischen Anordnung von Monitoren im Katheterlabor.

\subsubsection{Visuelle Fokussierung und Autonomie}

Es ist naheliegend, dass sich der Vorteil von GG aus der fest verankerten Position der Projektion im rechten oberen Quadranten des Benutzersichtfeldes sowie der selektiven Abrufbarkeit benötigter Informationen nach Bedarf und Ermessen des Operateurs während der PTA der A. femoralis superficialis erklären lässt. Bei der Bildgebung über mehrere Monitore, beziehungsweise über Breitbildmonitore mit mehreren eingeblendeten Fenstern, wird von dem Operateur ein weites Blickfeld mit entsprechender Übersicht über alle abgebildeten Informationen gefordert.

Periphere visuelle Eindrücke im Sichtfeld können jedoch eine Ablenkung darstellen (27). Die Blickrichtung und somit der visuelle Fokus auf den eigentlichen Ort der PTA (Schleuse am Simulator beziehungsweise LiveAngiografie-Monitor) wird hierbei unterbrochen, um die zusätzlich benötigten Informationen zu finden. Im Anschluss wird die Blickrichtung des Operateurs wieder neu ausgerichtet und damit der Fokus des Sichtfeldes erneut auf die PTA gelegt. GG ermöglicht hierbei die Abrufbarkeit benötigter Informationen per Sprachbefehl ohne eine Abwendung der Blickrichtung vom Operationsgebiet durch die Projektion im Sichtfeld. 
Diese Beobachtung entspricht ebenfalls den Ergebnissen von Vorraber et al. (27) und macht demnach wahrscheinlich auch den signifikanten zeitlichen Unterschied in unserer Studie aus.

\subsubsection{Bildauflösung, Bildkontrastierung und Farbsättigung}

Im Gegenzug war GG bei der Kontrolle der postinterventionellen Fluoroskopie (Tab.1, Aufg.5) dem klassischen Monitoring zeitlich unterlegen. Diese Aufgabe forderte den Teilnehmern ein genaues Überprüfen der gegebenen Grafik ab und testete damit die Wahrnehmung von Details im Vergleich beider bildgebender Verfahren. Hierdurch konnten einige der genannten technischen Grenzen von GG explizit aufgedeckt werden. Gruppe A war mutmaßlich durch die größere und schärfere Abbildung der Grafik über einen Monitor mit höherer Auflösung im Vorteil, welcher sich im zeitlichen Vergleich beider Gruppen signifikant erkennbar machte.

Die Fotografie aus Aufgabe 6 (Anh.10) wurde jedoch durch alle Probanden beider Gruppen korrekt als neu aufgetretene Ischämie des linken Fußes im Vergleich zum, postinterventionell gut durchbluteten, rechten Fuß interpretiert. Hierdurch konnte gezeigt werden, dass Abstufungen der Bildkontrastierung und Farbsättigung bei der Benutzung von GG (Gruppe B) genauso gut differenziert werden können, wie bei der Darstellung über einen Monitor (Gruppe $A$ ).

Die meisten interventionellen Rekanalisierung der A. femoralis superficialis erfolgen über einen arteriellen Zugang des kontralateralen Beines, um retrograd, über die Aortenbifurkation hinweg, in das arterielle System des betroffenen Beines zu gelangen. Dementsprechend kann es bei diesen sogenannten Cross-Over-PTAs als Komplikation auch zur Embolisation oder Dissektion von Gefäßen der gegenseitigen Extremität kommen (32). Die postinterventionelle klinische Kontrolle der Durchblutung beider Extremitäten nimmt demnach einen hohen Stellenwert ein.

Daraus schließen wir, dass GG bei der Unterscheidung von Farben und Kontrasten als gleichwertig, gegenüber der Darstellung über einen Monitor, anzusehen ist. 
Bei der Darstellung von grafischen Details jedoch ist man, vermutlich aufgrund der geringeren Auflösung und kleineren Abbildung der Grafiken über die Projektion von GG, gegenüber einem Monitor im Nachteil.

\subsubsection{Selektive Aufmerksamkeit}

Die Tatsache, dass die in Aufgabe 5 (Tab.1, Aufg.5) versteckte Patientenverwechslung sowohl in Gruppe A als auch in Gruppe B von weniger als einem Drittel bemerkt wurde, lässt sich am ehesten dem Phänomen der selektiven Aufmerksamkeit (33) zuordnen. Die Probanden konzentrierten sich demnach bei dieser Aufgabe überwiegend auf die korrekte Lage des zuvor eingebrachten Stents beziehungsweise auf das Ergebnis der bisher durchgeführten Intervention. Während der gesamten Simulation hatte die korrekte Seitenmarkierung von radiologischen Befunden oder die korrekten Personalien des Simulationspatienten (Anh.9) keine oder nur eine untergeordnete Relevanz. Diese Randinformationen wurden daher von mehr als zwei Dritteln der Probanden, unbewusst und zu Gunsten der Konzentration auf die PTA, aus dem Fokus der Aufmerksamkeit selektiert. Ein Unterschied im Vergleich beider bildgebender Verfahren lies sich demnach nicht beobachten, da es sich hierbei, unabhängig von der Form der Bildgebung, um ein individuelles Phänomen der Wahrnehmung eines jeden Probanden handelte.

\subsubsection{Komplexität visueller Informationen}

Die Lokalisierung der Pathologie in einer angiografischen Abbildung

(Tab.1, Aufg.2) wies seitens der abgebildeten Strukturen Ähnlichkeiten mit dem oben genannten Fluoroskopiebefund auf. Jedoch war diese Grafik wesentlich kontrastreicher und die Aufgabe auf die Lokalisation der deutlich sichtbaren, hochgradigen Stenose beschränkt (Anh.6).

In diesem Kontext relativierten sich die technischen Defizite von GG, wodurch kein zeitlicher Unterschied beider bildgebender Verfahren dokumentiert werden konnte. Abzüglich der App-Neustarts, wäre GG dem klassischen MonitorSetting im simulierten Katheterlabor bei dieser Aufgabe wahrscheinlich überlegen gewesen. 
Beim Lesen eines Textblocks, wie zum Beispiel einer patientenbezogenen Anamnese (Tab.1, Aufg.1), handelt es sich im engeren Sinne ebenfalls um eine detailreiche Grafik mit vielen kleinen Symbolen, welche das genaue Ablesen der einzelnen Wörter und Zeilen abverlangt.

Dementsprechend war GG hier wiederum unterlegen und könnte erst nach Abzug der benötigten Zeit für die App-Neustarts als gleichwertig gegenüber dem klassischen Monitoring angesehen werden. Es ist hervorzuheben, dass sich diese Aussage nur auf einen verhältnismäßig kleinen Textblock mit strukturiert dargestellten, kurzen Sätzen (Anh.5) im Rahmen der Studie beziehen lässt. Aufgrund der genannten Erfahrungen wäre GG bei einem komplexeren Textblock mutmaßlich im Nachteil.

\subsection{Ausblick auf heutige Systeme}

Nachdem sich GG als Pionier der oHMDs 2014 auf dem öffentlichen Markt etablierte, entwickelten auch weitere Unternehmen oHMDs, um ihre Vorstellung eines effektiven AR-Systems zu verwirklichen. Hierbei stellt die Verbesserung der digital ergänzten, visuellen Darstellungen im Sinne der AR einen der führenden Punkte zur Optimierung dieser Technologie dar.

Das Unternehmen Microsoft ermöglichte 2016 mit „HoloLens® 1“ (34) die Integration von dreidimensionalen Hologrammen in das reale Sichtfeld des Benutzers. Eine Besonderheit war zudem, dass HoloLens $\AA 1$ bestimmte Bewegungen und Gesten des Benutzers erkennt, um mit den besagten Hologrammen zu interagieren (35).

Aber nicht nur die Interaktion mit der erweiterten Realität, sondern auch die Integration der Hologramme in die reale Umgebung des Benutzers stellt eine Herausforderung der AR-Technologie dar.

Das 2010 gegründete Unternehmen Magic Leap vermarktete 2018 mit "MagicLeapOne®" (36) eine AR-Device, dass auf dem Prinzip der virtuellen Netzhautanzeige (engl.: virtual retinal display) aufbaut. 
Bei dieser Form der Abbildung wird das natürliche Lichtfeld, also die realen visuellen Informationen, die auf der Netzhaut des Auges auftreffen, von dem Device registriert und um ein digitales Lichtfeld auf der Netzhaut ergänzt.

So soll die Realität nicht nur durch dreidimensionale Hologramme erweitert, sondern für den Benutzer auch perspektivisch korrekt in den Raum integriert werden können (37).

Ein wichtiger Aspekt zur Etablierung von AR ist jedoch auch der Tragekomfort beziehungsweise das Aussehen der oHMDs. Es handelt sich hierbei um Eigenschaften der Hardware, die maßgeblich für die praktikable Nutzung im Alltag verantwortlich sind. Da die meisten oHMDs aufgrund der komplexen Technologie oftmals sehr unhandlich sind oder zumindest ungewöhnlich aussehen, fokussierte sich das Unternehmen Vuzix im Jahr 2018 vor allem auch auf das Design von „Vuzix Blade®“ (38).

Bei diesem Device handelt es sich um ein oHMD in Sonnenbrillenoptik, dass versucht diese Form von Technologie durch sein schlichtes Design unauffällig in den Alltag zu integrieren.

In diesem Zusammenhang sind auch sogenannte "smart contact lenses" zu erwähnen. Dabei handelt es sich um AR-Systeme in Form von Kontaktlinsen, welche aktuell Gegenstand technologischer Forschung sind (39).

Die ersten Prototypen, wie zum Beispiel „Mojo Lens®“ des Unternehmens Mojo Vision, sollen zurzeit bereits getestet werden (40).

Schließlich ist noch eine Kollaboration hervorzuheben, die nicht nur aufgrund ihrer Aktualität erwähnenswert ist, sondern zugleich den Kerngedanken unserer Studie stützt. Die Rede ist von einem Pilotprojekt zwischen Philips (41), einem der führenden Unternehmen in der Herstellung von Medizintechnik insbesondere auch im Kontext katheterbasierter Interventionen - und Microsoft mit der seit Ende 2019 auf dem Markt erhältlichen „HoloLens® ${ }^{\circledR}$ “ (42). 
Bereits im Frühjahr 2019 berichteten die beiden Unternehmen von der Erarbeitung eins Konzepts zur effektiven Verwendung von AR bei bildgestützten Verfahren in der Medizin. Es besteht Einigkeit zwischen beiden Firmen, dass AR in Zukunft, durch die intuitive und schnelle Informationsgewinnung über ein oHMD, einen Fortschritt in der Durchführung endovaskulärer Katheterinterventionen darstellen wird.

Die Verwendung interaktiver, dreidimensionaler Abbildungen soll hierbei die Präzision minimalinvasiver Eingriffe erhöhen, der Zugriff auf Patientendaten in Echtzeit und deren Einblendung im Sichtfeld des Operateurs zeitgleich den Fokus auf den Patienten verbessern (43). 


\section{SCHLUSSFOLGERUNG}

In Zusammenschau der Ergebnisse lässt sich feststellen, dass bei der Darstellung einfacher und überschaubarer Abbildungen, wie zum Beispiel beim Monitoring von Vitalparametern (Tab.1, Aufg.4), der schnelle und selektive Informationsgewinn, die Reduktion visueller Ablenkung sowie der konstante Fokus auf das Operationsgebiet bei der Benutzung von GG überwiegt, um gegenüber der klassischen Anordnung von Monitoren im Simulationskatheterlabor im Vorteil oder zumindest gleichwertig zu sein. Je komplexer und detailreicher jedoch die gewünschte grafische Darstellung ist, desto eher ist $G G$ aufgrund noch vorhandener technischer Defizite im Nachteil.

Wir sind der Meinung, dass durch die Behebung der derzeit noch vorhandenen technischen Mängel von GG kürzere Interventionszeiten erreicht werden könnten, da neben der herkömmlichen Datenübertragung zwei fundamentale Vorteile von oHMD bestehen:

Erstens kann sich der Operateur während endovaskulärer Interventionen stärker auf seine manuellen Fertigkeiten im Rahmen des Eingriffs konzentrieren und müsste während der Intervention nicht intermittierend seinen Blick vom OPGebiet abwenden, um die erforderlichen Informationen zu sehen.

Denn durch die Abrufbarkeit visueller Informationen direkt aus dem Sichtfeld des Operateurs, könnte die Anzahl der Bildschirme reduziert werden, was einer besseren Übersicht dient und damit eine geringere visuelle Ablenkung zur Folge hätte.

Vorraber et al. (27) haben bereits 2014 gezeigt, dass bei der Durchführung einer PTA eine deutliche Reduktion der perioperativen Kopfbewegungen durch die Benutzung von GG resultiert, als im Vergleich zur Durchführung derselben Intervention mit diversen Monitoren. Hierdurch konnte, durch die Verwendung von GG, die subjektive Konzentrationsfähigkeit der Operateure erhöht und gleichzeitig die Interventionszeiten verkürzt werden.

Unsere eigene Studie konnte zeigen, dass visuelle Informationsquellen während endovaskulärer Eingriffe auf die Live-Angiografie-Monitore und die Verwendung eines oHMD reduziert werden können, ohne dass dies Nachteile in Bezug auf die Qualität und den zeitlichen Ablauf des Verfahrens haben muss. 
Zweitens können sich Operateure, unter Verwendung eines oHMD, visuelle Informationen autonom zugänglich machen. Die Sterilitätskriterien in Operationssälen machen es den Operateuren normalerweise unmöglich, die Bildschirme selbstständig zu bedienen und anzupassen. Der Operateur ist demnach weitestgehend auf die Unterstützung eines weiteren Mitarbeiters angewiesen. In unserer Studie wurde diese Rolle vom Studienleiter an zuvor festgelegten Kontrollpunkten übernommen.

Im Hinblick auf reale Situationen, in denen das OP-Personal den Informationsbedarf des Operateurs nicht kennt, solange darüber nicht kommuniziert wird, kann dies mit einem weiteren Zeitverlust einhergehen und zu einer potentiellen Gefahrenquelle durch fehlerhafte Kommunikation werden.

Lingard et al. (44) beobachteten Kommunikationsfehler in etwa $30 \%$ des verfahrensrelevanten Informationsaustauschs zwischen Mitgliedern von OPTeams, woraus in $17,8 \%$ ein ineffizientes intraoperatives Vorgehen resultierte. $60 \%$ aller unserer Probanden stimmten zu, dass Missverständnisse in der Kommunikation zu Verzögerungen im Interventionsablauf führen können.

Diese Ansicht deckt sich mit den Ergebnissen von Doepfer et al. (45) und Seemann et al. (46), welche den Faktor Mensch, unter anderem aufgrund von mangelnder Kommunikation und schlechter Teamarbeit, als eine der größten Fehlerquellen im medizinischen Alltag aufgriffen und die Notwendigkeit des Trainings interpersoneller Kompetenzen, in Anlehnung an die Standards der Ausbildung in der Luftfahrt, auch in der Chirurgie etablierten. Eine weitere Parallele zur Ausbildung in der Luftfahrt griffen Mitha et al. (47) auf. Sie berichteten in ihrer Studie von Simulationen mit VR- oder AR-Technologie, unter anderem auch den von uns verwendeten ANGIO-Mentor ${ }^{\circledR}$, als geeignetes Mittel zur risikofreien Übung und Vorbereitung endovaskulärer Interventionen.

$82,5 \%$ der Probanden unserer Studie glauben in diesem Zusammenhang an eine mögliche Zeitersparnis durch den Einsatz moderner Technologie in Katheterlaboren. Die kombinierte Verwendung von Spracherkennung und oHMD erhöht somit die Unabhängigkeit des Operateurs von anderen Mitarbeitern durch autonome und selektive Informationsgewinnung. 65\% unserer Teilnehmer empfanden dies als einen positiven Aspekt im Rahmen unserer simulierten PTA der A. femoralis superficialis. 
Nichtsdestotrotz ist die persönliche Interaktion zwischen allen Beteiligten im OP-Saal eine wertvolle Eigenschaft des Teams und kann wahrscheinlich nicht durch eine Form von Technologie ersetzt, aber sehr wohl unterstützt werden. 90\% unserer Teilnehmer halten die Möglichkeit mit dem OP-Personal kommunizieren zu können in diesem Kontext für wichtig und hilfreich.

Basierend auf unserer Erfahrung während der Studie, könnte GG eine supportive Funktion während peripheren endovaskulären Interventionen einnehmen, indem sie dem Operateur als Bindeglied für einen individualisierten und effizienten Informationsfluss dient und gleichzeitig das übrige Personal in seinen Aufgaben entlastet. Zusammenfassend könnte die Integration eines sprachgesteuerten Bildgebungssystems zu einer effizienteren Aufgabenverteilung in den Operationssälen führen. 


\section{FAZIT}

GG behinderte den Arbeitsablauf während der simulierten PTA einer hochgradigen A. femoralis superficialis Stenose nicht und konnte im Umfeld eines VR-Katheterlabors sinnvoll eingesetzt werden. Die Erfahrung mit einem sprachgesteuerten oHMD war überwiegend positiv. Das Gerät konnte den Benutzer, in Kombination mit der Custom Glass App, nicht nur dabei unterstützen sich auf die aktuell benötigten Informationen zu konzentrieren, sondern auch die autonome Kontrolle des Datenflusses ermöglichen.

Hinsichtlich der Interventionsdauer war GG der klassischen Anordnung von Monitoren im Simulationskatheterlabor jedoch zeitlich geringfügig unterlegen. Unsere Studie führte diese Beobachtung auf technische Schwierigkeiten hinsichtlich der Genauigkeit der Spracherkennung und der Bildqualität zurück. Wir erwarten, dass diese technischen Probleme durch eine überarbeitete Custom Glass App und die Benutzung eines weiterentwickelten oHMD überwunden, und somit der Arbeitsablauf im Katheterlabor während peripheren endovaskulären Eingriffen weiter verbessert werden kann.

Möglicherweise ist dies bereits teilweise durch die Verwendung, der im Sommer 2017 herausgebrachten, neueren Version von GG, der Google Glass $®$

Enterprise Edition (48), möglich. Hierbei handelt es sich um eine, anhand der verschiedenen Erfahrungen von Unternehmen in diversen Wirtschaftszweigen, optimierte Version der GG Explorer Edition, die zuletzt 2019 technisch verbessert (49) als Google Glass $®$ Enterprise Edition 2 (50) auf den Markt kam. Diese sollte im Rahmen weiterer Studien unter Verwendung einer überarbeiteten Custom Glass App und unter realen Operationsbedingungen von erfahrenen Operateuren getestet werden.

Ob sich AR-Systeme, wie oHMDs, bei peripheren endovaskulären Eingriffen oder auch in anderen medizinischen Bereichen tatsächlich etablieren werden, bleibt daher abzuwarten. Jedoch zeigt das bisherige Interesse verschiedener Unternehmen sowie deren technologischen Entwicklungen der letzten 5 Jahre (5.4), dass dieser Markt im Sinne unserer Studie auch in Zukunft noch großes Potenzial birgt. 


\section{LITERATURVERZEICHNIS}

1. Bundesnetzagentur. Digitale Transformation in den Netzsektoren 2017 [cited 2020 May 15]. Available from: URL:

https://www.bundesnetzagentur.de/SharedDocs/Downloads/DE/Sachgeb iete/Telekommunikation/Unternehmen_Institutionen/Digitalisierung/Grun dsatzpapier/Digitalisierung.pdf?_blob=publicationFile\&v=3.

2. Reith R, Scarsella A, Shirer M. Flat Smartphone Growth Projected for 2016 as Mature Markets Veer into Declines, According to IDC; 2016 [cited 2020 May 15]. Available from: URL: https://www.businesswire.com/ news/home/20160901005130/en/Flat-Smartphone-Growth-Projected2016-Mature-Markets.

3. Oculus VR LLC. Oculus; 2017 [cited 2020 May 15]. Available from: URL: https://www.oculus.com/rift-s/.

4. HTC Corporation. VIVE ${ }^{\mathrm{TM}}$ | Discover Virtual Reality Beyond Imagination; 2018 [cited 2020 May 15]. Available from: URL: https://www.vive.com/us/.

5. Sony Interactive Entertainment Europe Limited. PlayStation®VR; 2018 [cited 2020 May 15]. Available from: URL: https://www.playstation.com/en-gb/explore/playstation-vr/.

6. Augmedix Inc. Rehumanizing Healthcare; 2018 [cited 2020 May 15]. Available from: URL: https://www.augmedix.com/.

7. Google Inc. Tech specs - Google Glass Help; 2017 [cited 2020 May 15]. Available from: URL: https://support.google.com/ glass/answer/3064128?hl=en\&ref_topic=3063354 .

8. Torborg S, Simpson S. Google Glass Teardown: What's inside Google Glass?; 2014 [cited 2020 May 15]. Available from: URL: http://www.catwig.com/google-glass-teardown/.

9. Liebert CA, Zayed MA, Aalami O, Tran J, Lau JN. Novel Use of Google Glass for Procedural Wireless Vital Sign Monitoring. Surg Innov 2016 [cited 2020 May 15]; 23(4):366-73. 
10. Rahimy E, Garg SJ. Google Glass for Recording Scleral Buckling Surgery. JAMA Ophthalmology 2015 [cited 2020 May 15]; 133(6):710-1. Available from: URL: http://jamanetwork.com/ journals/jamaophthalmology/article-abstract/2210534.

11. Diaz R, Yoon J, Chen R, Quinones-Hinojosa A, Wharen R, Komotar R. Real-time Video-Streaming to Surgical Loupe Mounted Head-Up Display for Navigated Meningioma Resection. Turk Neurosurg 2017 [cited 2020 May 15]; 27(4):1-7.

12. Jeroudi OM, Christakopoulos G, Christopoulos G, Kotsia A, Kypreos MA, Rangan BV et al. Accuracy of remote electrocardiogram interpretation with the use of Google Glass technology. Am J Cardiol 2015 [cited 2020 May 15]; 115(3):374-7.

13. Albrecht U-V, Jan U von, Kuebler J, Zoeller C, Lacher M, Muensterer OJ et al. Google Glass for documentation of medical findings: Evaluation in forensic medicine. J Med Internet Res 2014 [cited 2020 May 15]; 16(2):e53.

14. Chai PR, Wu RY, Ranney ML, Bird J, Chai S, Zink B et al. Feasibility and Acceptability of Google Glass for Emergency Department Dermatology Consultations. JAMA Dermatol 2015 [cited 2020 May 15]; 151(7):794-6.

15. Chaballout B, Molloy M, Vaughn J, Brisson lii R, Shaw R. Feasibility of Augmented Reality in Clinical Simulations: Using Google Glass With Manikins. JMIR Med Educ 2016 [cited 2020 May 15]; 2(1):e2.

16. Vávra $P$, Roman J, Zonča $P$, Innát $P$, Němec $M$, Kumar $J$ et al. Recent Development of Augmented Reality in Surgery: A Review. J Healthc Eng 2017 [cited 2020 May 15]; 2017:4574172.

17. Grinshpoon A, Sadri S, Loeb GJ, Elvezio C, Siu S, Feiner SK. Handsfree augmented reality for vascular interventions. In: 2018 - ACM SIGGRAPH 2018 Emerging Technologies. p. 1-2 [cited 2020 May 15]. 
18. Lu W, Wang L, Zhou W, Fu W. Augmented reality navigation to assist retrograde peroneal access for the endovascular treatment of critical limb ischemia. J Vasc Surg Cases Innov Tech 2019 [cited 2020 May 15]; 5(4):518-20.

19. Yoon JW, Chen RE, Han PK, Si P, Freeman WD, Pirris SM. Technical feasibility and safety of an intraoperative head-up display device during spine instrumentation. Int J Med Robot 2016 [cited 2020 May 15].

20. Drake-Brockman TFE, Datta A, Ungern-Sternberg BS von. Patient monitoring with Google Glass: A pilot study of a novel monitoring technology. Paediatr Anaesth 2016 [cited 2020 May 15]; 26(5):539-46.

21. Aungst TD, Lewis TL. Potential uses of wearable technology in medicine: Lessons learnt from Google Glass. Int J Clin Pract 2015 [cited 2020 May 15]; 69(10):1179-83.

22. Mishra S. Google Glass in medicine - The man with a computer on face. Indian Heart J 2016 [cited 2020 May 15]; 68(4):586-7.

23. Chang JYC, Tsui LY, Yeung KSK, Yip SWY, Leung GKK. Surgical Vision: Google Glass and Surgery. Surg Innov 2016 [cited 2020 May 15]; 23(4):422-6.

24. Muensterer OJ, Lacher M, Zoeller C, Bronstein M, Kubler J. Google Glass in pediatric surgery: An exploratory study. Int J Surg 2014 [cited 2020 May 15]; 12(4):281-9.

25. Borgmann H, Rodríguez Socarrás M, Salem J, Tsaur I, Gomez Rivas J, Barret $E$ et al. Feasibility and safety of augmented reality-assisted urological surgery using smartglass. World J Urol 2016 [cited 2020 May 15]; 35(6):967-72.

26. Madsack B, Walz M, Weisser G. Abnahme und Konstanzprüfung an Bildwiedergabesystemen - was ändert sich mit der neuen DIN V 6868157? Radiopraxis 2014; 7(04):195-210.

27. Vorraber W, Voessner S, Stark G, Neubacher D, DeMello S, Bair A. Medical applications of near-eye display devices: An exploratory study. Int J Surg 2014 [cited 2020 May 15]; 12(12):1266-72. 
28. Google Inc. Frames - Google Glass; 2017 [cited 2020 May 15]. Available from: URL: https://www.google.com/glass/help/frames/.

29. Halford GS, Baker R, McCredden JE, Bain JD. How many variables can humans process? Psychol Sci 2005 [cited 2020 May 15]; 16(1):70-6.

30. Cowan N. The magical number 4 in short-term memory: A reconsideration of mental storage capacity. Behav Brain Sci 2001 [cited 2020 May 15]; 24(1):87-114; discussion 114-85.

31. Hemond C, Brown RM, Robertson EM. A distraction can impair or enhance motor performance. J Neurosci 2010 [cited 2020 May 15]; 30(2):650-4.

32. Gjeka R. Interventional Strategies for the Superficial Femoral Artery. In: Sareen N, Ojha A, editors. Peripheral Arterial Disease - A Practical Approach: InTech; 2018. p. 9-25 [cited 2020 May 15].

33. Moore T, Zirnsak M. Neural Mechanisms of Selective Visual Attention. Annu Rev Psychol 2017 [cited 2020 May 15]; 68:47-72.

34. Microsoft Corporation. HoloLens-Hardware (1. Generation); 2019 [cited 2020 May 13]. Available from: URL: https://docs.microsoft.com/dede/hololens/hololens1-hardware.

35. Microsoft Corporation. Getting around HoloLens (1st gen); 2019 [cited 2020 May 15]. Available from: URL: https://docs.microsoft.com/enus/hololens/hololens1-basic-usage.

36. Magic Leap. Magic Leap 1: A thousand breakthroughs in one; 2018 [cited 2020 May 14]. Available from: URL: https://www.magicleap.com/en-us/magic-leap-1.

37. Greenlight Insights. Magic Leap Reveals Smart Glasses: Shows Future of Spatial Computing 2017 [cited 2020 May 15].

38. Vuzix. Vuzix Blade: Powering Solutions; 2018 [cited 2020 May 15]. Available from: URL: https://www.vuzix.com/products/blade. 
39. Park J, Ahn DB, Kim J, Cha E, Bae B-S, Lee S-Y et al. Printing of wirelessly rechargeable solid-state supercapacitors for soft, smart contact lenses with continuous operations. Sci. Adv. 2019; 5(12):eaay0764.

40. Mojo Vision Inc. Mojo Lens | The World's First True Smart Contact Lens; 2020 [cited 2020 May 13]. Available from: URL: https://www.mojo.vision/mojo-lens.

41.Philips $\mathrm{GmbH}$. Philips Azurion: Performance und optimale Versorgung [Philips Healthcare]; 2017 [cited 2020 May 15]. Available from: URL: https://www.philips.de/healthcare/resources/landing/azurion?origin=7_70 0000001848547_71700000067139629_58700005873131935_43700053 682874380\&dmcm=EAlalQobChMI67qPrKKv6QIVzed3ChOuegf8EAAYA SAAEgIZf_D_BwE\&gclid=EAlalQobChMI67qPrKKv6QIVzed3Ch0uegf8E AAYASAAEgIZf_D_BwE\&gclsrc=aw.ds.

42. Microsoft Corporation. HoloLens 2: Get to know the features and technical specs; 2019 [cited 2020 May 13]. Available from: URL: https://www.microsoft.com/en-us/hololens/hardware.

43. Philips $\mathrm{GmbH}$. Philips and Microsoft HoloLens 2: Could augmented reality change the face of image guided therapy?; 2019 [cited 2020 May 15].

44. Lingard L. Communication failures in the operating room: An observational classification of recurrent types and effects. Quality and Safety in Health Care 2004 [cited 2020 May 15]; 13(5):330-4.

45. Doepfer A-K, Seemann R, Merschin D, Stange R, Egerth M, Münzberg M et al. Sicherheitskultur in Orthopädie und Unfallchirurgie: Kurskonzept: Interpersonal Competence by Deutsche Gesellschaft für Orthopädie und Unfallchirurgie (DGOU) and Lufthansa Aviation Training. Ophthalmologe 2017 [cited 2020 May 15]; 114(10):890-3.

46. Seemann R, Münzberg M, Stange R, Rüsseler M, Egerth M, Bouillon B et al. Interpersonelle Kompetenz in Orthopädie und Unfallchirurgie: Warum technische und prozedurale Fähigkeiten alleine nicht ausreichen. Unfallchirurg 2016 [cited 2020 May 15]; 119(10):881-4. 
47. Mitha AP, Almekhlafi MA, Janjua MJJ, Albuquerque FC, McDougall CG. Simulation and augmented reality in endovascular neurosurgery: Lessons from aviation. Neurosurgery 2013 [cited 2020 May 15]; 72 Suppl 1:107-14.

48. Kothari J(Google Inc.). A new chapter for Glass: Medium Corp.; 2017 [cited 2020 May 15]. Available from: URL: https://blog.x.company/a-newchapter-for-glass-c7875d40bf24.

49. Google Inc. Tech Specs - Glass Enterprise Edition 2; 2019 [cited 2020 May 15]. Available from: URL: https://www.google.com/glass/tech-specs/.

50. Kothari J(Google Inc.). Glass Enterprise Edition 2: faster and more helpful: Project Lead Glass; 2019 [cited 2020 May 15]. Available from: URL: https://www.blog.google/products/hardware/glass-enterpriseedition-2/. 


\section{ANHANG}

Anhang 1 Einverständniserklärung zur Verwendung der Daten.................. XIX

Anhang 2 Standardisiertes Bewertungsprotokoll.................................. XX

Anhang 3 Fragebogen Gruppe A (Kontrollgruppe) ............................ XXI

Anhang 4 Fragebogen Gruppe B (Experimentalgruppe).......................XXIII

Anhang 5 Custom Glass App-Screenshot: Aufgabe 1 (Anamnese)...........XXV

Anhang 6 Custom Glass App-Screenshot: Aufgabe 2 (Angiografie)..........XXVI

Anhang 7 Custom Glass App-Screenshot: Aufgabe 3 (Laborwerte)......... XXVII

Anhang 8 Custom Glass App-Screenshot: Aufgabe 4 (Vitalparameter)...XXVIII

Anhang 9 Custom Glass App-Screenshot: Aufgabe 5 (Fluoroskopie)........XXIX

Anhang 10 Custom Glass App-Screenshot: Aufgabe 6 (Foto)...................XXX 


\title{
Anhang 1 Einverständniserklärung zur Verwendung der Daten
}

\author{
"Kann Google Glass $®$ den Arbeitsablauf endovaskulärer Interventionen \\ verbessern? Eine Pilotstudie mit Google Glass $®$ im Rahmen einer Virtual \\ Reality Simulation."
}

\section{Einverständniserklärung}

Hiermit erkläre ich, dass ich über die Ziele, den Ablauf und über die Dauer und den Nutzen der Studie ausführlich und verständlich mündlich aufgeklärt worden bin. Ich habe die Entscheidung zur Studienteilnahme aus freiem Willen getroffen und alle meine Fragen sind zu meiner Zufriedenheit beantwortet worden.

Mir ist bekannt, dass bei der Studie personenbezogene Daten erhoben, gespeichert und ausgewertet werden sollen. Ich bin damit einverstanden, dass der Projektleiter Dennis Kaczmarek, sowie mit der Studie vertraute Mitarbeiter/innen, Einblick in meine Daten nehmen können.

Ich stimme zu, dass die erhobenen Daten unter der Verantwortung des Projektleiters in anonymisierter Form für die wissenschaftlichen Fragestellungen der Studie gespeichert, verarbeitet und publiziert werden.

Mir ist bekannt, dass ich jederzeit und ohne Angabe von Gründen die Einwilligung zur Verwendung meiner personenbezogenen Daten zurückziehen und deren Löschung verlangen kann, insofern diese noch nicht anonymisiert worden sind.

\section{Ich erkläre mich bereit, an der o. g. Studie freiwillig teilzunehmen.}

Proband/in (Name, Vorname in Druckbuchstaben) 


\section{Anhang 2 Standardisiertes Bewertungsprotokoll}

Jede durchgeführte Aufgabe bzw. korrekte Aussage wird mit jeweils einem Punkt bewertet. Es wird sowohl die benötigte Zeit pro Aufgabe, als auch die Gesamtbearbeitungszeit dokumentiert. Die Anzahl etwaiger App-Neustarts in Gruppe $B$ wird ebenfalls notiert und als Zeitverlust aufgrund von Neustarts vermerkt.

Proband (Unzutreffendes streichen): männlich / weiblich Gruppe A (Monitor) / Gruppe B (Google Glass $®$ )

\begin{tabular}{|c|c|c|c|}
\hline Aufgabe & $\begin{array}{l}\text { Durchgeführt } \\
\quad \text { (Punkt) }\end{array}$ & $\begin{array}{c}\text { Zeit } \\
\text { (min:sek) }\end{array}$ & $\begin{array}{c}\text { App- } \\
\text { Neustarts } \\
\text { (Gruppe B) }\end{array}$ \\
\hline \multicolumn{4}{|l|}{ 1. Anamnese } \\
\hline \multicolumn{4}{|l|}{$\begin{array}{l}\text { Verdachtsdiagnose pAVK gestellt bzw. } \\
\text { arterielle Stenose des rechten Beins } \\
\text { richtig erkannt? }\end{array}$} \\
\hline \multicolumn{4}{|l|}{ 2. Angiografie } \\
\hline \multicolumn{4}{|l|}{$\begin{array}{l}\text { Stenose erkannt, lokalisiert und ggf. } \\
\text { benannt (rechte A. femoralis } \\
\text { superficialis)? }\end{array}$} \\
\hline \multicolumn{4}{|l|}{ 3. Laborwerte } \\
\hline \multicolumn{4}{|l|}{$\begin{array}{l}\text { Pathologische Werte genannt? } \\
(\mathrm{LDL} \uparrow \mathrm{TSH} \downarrow \mathrm{TT} 3+\mathrm{fT} 4 \uparrow)\end{array}$} \\
\hline \multicolumn{4}{|l|}{ Relevanz der Hyperthyreose erkannt? } \\
\hline \multicolumn{4}{|l|}{ Relevanz der Nierenfunktion erkannt? } \\
\hline \multicolumn{4}{|l|}{ 4. Vitalparameter } \\
\hline \multicolumn{4}{|l|}{$\begin{array}{l}\text { Kritische Werte erkannt und benannt? } \\
(\mathrm{RR} \downarrow \mathrm{HF} \uparrow)\end{array}$} \\
\hline \multicolumn{4}{|l|}{ 5. Fluoroskopie } \\
\hline \multicolumn{4}{|l|}{$\begin{array}{l}\text { Freies arterielles System mit einseitigem } \\
\text { Stent-Graft erkannt? }\end{array}$} \\
\hline \multicolumn{4}{|l|}{$\begin{array}{l}\text { Falschen Patienten bzw. Stent auf der } \\
\text { falschen Seite bemerkt? }\end{array}$} \\
\hline \multicolumn{4}{|l|}{ 6. Fotodokumentation } \\
\hline \multicolumn{4}{|l|}{$\begin{array}{l}\text { Minderdurchblutung bzw. Blässe } \\
\text { erkannt? }\end{array}$} \\
\hline $\begin{array}{l}\text { Verdachtsdiagnose eines kontralateralen } \\
\text { Rezidivs gestellt (pAVK bzw. Stenose)? }\end{array}$ & & & \\
\hline
\end{tabular}

\begin{tabular}{|c|c|c|}
\hline $\begin{array}{c}\text { Punktzahl } \\
\text { (insgesamt) }\end{array}$ & $\begin{array}{c}\text { Gesamtzeit } \\
\text { (min:sek) }\end{array}$ & $\begin{array}{c}\text { Zeitverlust } \\
\text { (min:sek) }\end{array}$ \\
\hline & & \\
\hline
\end{tabular}




\section{Anhang 3 Fragebogen Gruppe A (Kontrollgruppe)}

\section{Angaben zur Person}

Alter

Jahre

Semester

Geschlecht

männlich

weiblich

Bitte bewerten Sie die folgenden Fragen auf einer Skala von 1 bis 5

(1 = Trifft vollständig zu, 5 = Trifft überhaupt nicht zu)

\begin{tabular}{|c|c|c|c|c|c|}
\hline Folgende Aussagen treffen zu & Trifft v & ig zu & & Trifft übe & hicht zu \\
\hline & 1 & 2 & 3 & 4 & 5 \\
\hline $\begin{array}{l}\text { Ich empfinde es als hilfreich jederzeit in } \\
\text { Kommunikation mit der OP-Schwester treten } \\
\text { zu können. }\end{array}$ & $\square$ & $\square$ & $\square$ & $\square$ & $\square$ \\
\hline $\begin{array}{l}\text { Ich empfinde es als stressig während einer } \\
\text { Intervention parallel für Kollegen Frage und } \\
\text { Antwort zu stehen. }\end{array}$ & $\square$ & $\square$ & $\square$ & $\square$ & $\square$ \\
\hline $\begin{array}{l}\text { Ich fühle mich durch die Interaktion mit } \\
\text { Kollegen während einer Intervention in meiner } \\
\text { Arbeit eingeschränkt (Tempo, Qualität, } \\
\text { Endergebnis etc.). }\end{array}$ & $\square$ & $\square$ & $\square$ & $\square$ & $\square$ \\
\hline $\begin{array}{l}\text { Ich arbeite gerne unabhängig und nach } \\
\text { eigenem strukturellem Vorgehen. }\end{array}$ & $\square$ & $\square$ & $\square$ & $\square$ & $\square$ \\
\hline $\begin{array}{l}\text { Ich bevorzuge die Verwendung analoger } \\
\text { Medien (sprich: ausgedruckter, greifbarer } \\
\text { Medien, wie z.B. Anamnesebögen, Fotos etc.) } \\
\text { gegenüber digitalem Material (über Monitore } \\
\text { eingeblendete Informationen). }\end{array}$ & $\square$ & $\square$ & $\square$ & $\square$ & $\square$ \\
\hline $\begin{array}{l}\text { Ich schätze den Informationsgewinn aus } \\
\text { digitalen Medien höher ein als vergleichbares } \\
\text { Material in analoger Form (z.B. Röntgenbild: } \\
\text { Monitor/Beamer vs. klassischer Röntgenfilm). }\end{array}$ & $\square$ & $\square$ & $\square$ & $\square$ & $\square$ \\
\hline $\begin{array}{l}\text { Ich sehe die Digitalisierung im klinischen } \\
\text { Alltag als vorteilhaft und sinnvoll. }\end{array}$ & $\square$ & $\square$ & $\square$ & $\square$ & $\square$ \\
\hline
\end{tabular}




\begin{tabular}{|c|c|c|c|c|c|}
\hline \multirow[t]{2}{*}{ Folgende Aussagen treffen zu } & \multicolumn{3}{|c|}{ Trifft vollständig zu } & \multicolumn{2}{|c|}{ Trifft überhaupt nicht zu } \\
\hline & 1 & 2 & 3 & 4 & 5 \\
\hline $\begin{array}{l}\text { Ich bin der Auffassung, dass es durch } \\
\text { fehlerhafte Kommunikation im HKL oft zu } \\
\text { Verzögerungen im Interventionsablauf } \\
\text { kommt. }\end{array}$ & $\square$ & $\square$ & $\square$ & $\square$ & $\square$ \\
\hline $\begin{array}{l}\text { Ich bin der Meinung, dass die } \\
\text { Interventionsdauer im HKL nicht relevant } \\
\text { verkürzt werden kann ohne Nachteile im } \\
\text { Patienten-Ergebnis hervorzurufen. }\end{array}$ & $\square$ & $\square$ & $\square$ & $\square$ & $\square$ \\
\hline $\begin{array}{l}\text { Ich bin der Meinung, dass durch den Einsatz } \\
\text { weiterer digitaler Medien eine Zeitersparnis } \\
\text { im HKL erreichbar ist. }\end{array}$ & $\square$ & $\square$ & $\square$ & $\square$ & $\square$ \\
\hline $\begin{array}{l}\text { Das Prinzip der digital „erweiterten Realität“ } \\
\text { (Augmented Reality) ist mir bekannt. }\end{array}$ & & & & & \\
\hline $\begin{array}{l}\text { Wenn JA: Ich kann mir prinzipiell vorstellen, } \\
\text { dass „Augmented Reality“ im HKL sinnvoll } \\
\text { eingesetzt werden kann. }\end{array}$ & $\square$ & $\square$ & $\square$ & $\square$ & $\square$ \\
\hline
\end{tabular}

\section{Anmerkungen, Kommentare, Kritik ?}

Vielen Dank, dass Sie sich die Zeit genommen haben! 


\section{Anhang 4 Fragebogen Gruppe B (Experimentalgruppe)}

\section{Angaben zur Person}

Alter

Jahre

Semester

Geschlecht

männlich

weiblich

Bitte bewerten Sie die folgenden Fragen auf einer Skala von 1 bis 5

(1 = Trifft vollständig zu, 5 = Trifft überhaupt nicht zu)

\begin{tabular}{|c|c|c|c|c|c|}
\hline \multirow[t]{2}{*}{ Folgende Aussagen treffen zu } & \multicolumn{3}{|c|}{ Trifft vollständig zu } & \multicolumn{2}{|c|}{ Trifft überhaupt nicht zu } \\
\hline & 1 & 2 & 3 & 4 & 5 \\
\hline $\begin{array}{l}\text { Ich empfinde es als hilfreich jederzeit in } \\
\text { Kommunikation mit der OP-Schwester treten } \\
\text { zu können. }\end{array}$ & $\square$ & $\square$ & $\square$ & $\square$ & $\square$ \\
\hline $\begin{array}{l}\text { Ich empfinde es als stressig während einer } \\
\text { Intervention parallel für Kollegen Frage und } \\
\text { Antwort zu stehen. }\end{array}$ & $\square$ & $\square$ & $\square$ & $\square$ & $\square$ \\
\hline $\begin{array}{l}\text { Ich fühle mich durch die Interaktion mit } \\
\text { Kollegen, während einer Intervention in meiner } \\
\text { Arbeit eingeschränkt (Tempo, Qualität, } \\
\text { Endergebnis etc.). }\end{array}$ & $\square$ & $\square$ & $\square$ & $\square$ & $\square$ \\
\hline $\begin{array}{l}\text { Ich arbeite gerne unabhängig und nach } \\
\text { eigenem strukturellem Vorgehen. }\end{array}$ & $\square$ & $\square$ & $\square$ & $\square$ & $\square$ \\
\hline $\begin{array}{l}\text { Ich bevorzuge die Verwendung analoger } \\
\text { Medien (sprich: ausgedruckter, greifbarer } \\
\text { Medien, wie z.B. Anamnesebögen, Fotos etc.) } \\
\text { gegenüber digitalem Material (über Monitore } \\
\text { eingeblendete Informationen). }\end{array}$ & $\square$ & $\square$ & $\square$ & $\square$ & $\square$ \\
\hline $\begin{array}{l}\text { Ich schätze den Informationsgewinn aus } \\
\text { digitalen Medien höher ein als vergleichbares } \\
\text { Material in analoger Form (z.B. Röntgenbild: } \\
\text { Monitor/Beamer vs. klassischer Röntgenfilm). }\end{array}$ & $\square$ & $\square$ & $\square$ & $\square$ & $\square$ \\
\hline $\begin{array}{l}\text { Ich sehe die Digitalisierung im klinischen } \\
\text { Alltag als vorteilhaft und sinnvoll. }\end{array}$ & $\square$ & $\square$ & $\square$ & $\square$ & $\square$ \\
\hline
\end{tabular}




\begin{tabular}{|l|cccc|}
\hline Folgende Aussagen treffen zu & Trifft vollständig zu & & Trifft überhaupt nicht zu \\
\hline & 1 & 2 & 3 & 4 \\
\hline $\begin{array}{l}\text { Ich bin der Auffassung, dass es durch } \\
\text { fehlerhafte Kommunikation im HKL oft zu } \\
\text { Verzögerungen im Interventionsablauf } \\
\text { kommt. }\end{array}$ & $\square$ & $\square$ & $\square$ & $\square$ \\
\hline & & $\square$ & $\square$ & $\square$ \\
\hline $\begin{array}{l}\text { Ich bin der Meinung, dass die } \\
\text { Interventionsdauer im HKL nicht relevant } \\
\text { verkürzt werden kann ohne Nachteile im } \\
\text { Patienten-Ergebnis hervorzurufen. }\end{array}$ & $\square$ & $\square$ & $\square$ & $\square$ \\
\hline & & & \\
\hline $\begin{array}{l}\text { Ich bin der Meinung, dass durch den Einsatz } \\
\text { weiterer digitaler Medien eine Zeitersparnis } \\
\text { im HKL erreichbar ist. }\end{array}$ & $\square$ & $\square$ & $\square$ \\
\hline & & & \\
\hline $\begin{array}{l}\text { Das Prinzip der digital „erweiterten Realität“ } \\
\text { (Augmented Reality) ist mir bekannt. } \\
\begin{array}{l}\text { Wenn JA: Ich kann mir prinzipiell vorstellen, } \\
\text { dass „Augmented Reality“ im HKL sinnvoll } \\
\text { eingesetzt werden kann. }\end{array}\end{array}$ & $\square$ & $\square$ & $\square$ & $\square$ \\
\hline
\end{tabular}

\begin{tabular}{|c|c|c|c|c|c|}
\hline $\begin{array}{l}\text { Die Handhabung von Google Glass } ₫ \text { fiel } \\
\text { mir... }\end{array}$ & $\begin{array}{c}\text { leicht } \\
\square\end{array}$ & $\square$ & $\square$ & $\square$ & $\begin{array}{c}\text { schwer } \\
\square\end{array}$ \\
\hline $\begin{array}{l}\text { Die Benutzung eines sprachgesteuerten } \\
\text { Systems empfand ich als... }\end{array}$ & $\begin{array}{l}\text { leicht } \\
\square\end{array}$ & $\square$ & $\square$ & $\square$ & $\begin{array}{l}\text { schwer } \\
\square\end{array}$ \\
\hline $\begin{array}{l}\text { Ich empfand das tragen von Google } \\
\text { Glass } ® \text { als... }\end{array}$ & $\begin{array}{l}\text { störend } \\
\square\end{array}$ & $\square$ & $\square$ & $\square$ & $\begin{array}{l}\text { angenehm } \\
\square\end{array}$ \\
\hline $\begin{array}{l}\text { Ich fühlte mich beim Tragen von } \\
\text { Google Glass } ® \text { in meinem Sichtfeld... }\end{array}$ & ergänzt & $\square$ & $\square$ & $\square$ & $\stackrel{\text { begrenzt }}{\square}$ \\
\hline $\begin{array}{l}\text { Die Qualität der optischen Informationen } \\
\text { durch Google Glass } ® \text { war... }\end{array}$ & gut & $\square$ & $\square$ & $\square$ & $\begin{array}{c}\text { schlecht } \\
\square\end{array}$ \\
\hline $\begin{array}{l}\text { Ich kann mir vorstellen, dass Google } \\
\text { Glass } ₫ \text { eine Zukunft im HKL hat.... }\end{array}$ & gut & $\square$ & $\square$ & $\square$ & $\begin{array}{c}\text { schlecht } \\
\square\end{array}$ \\
\hline
\end{tabular}

\section{Anmerkungen, Kommentare, Kritik ?}

\section{Vielen Dank, dass Sie sich die Zeit genommen haben!}


Anhang 5 Custom Glass App-Screenshot: Aufgabe 1 (Anamnese)

\begin{tabular}{|c|c|}
\hline \multirow{2}{*}{$\begin{array}{l}\text { Patient: } \\
\text { Vorerkrankungen }\end{array}$} & Stefan Pavkoff, 73 Jahre alt \\
\hline & $\begin{array}{l}\text { Diabetes mellitus Typ II, Hypertonie, } \\
\text { Hypercholesterinämie Adipositas Grad } \amalg(\mathrm{BMI}=38)\end{array}$ \\
\hline Anamnese: & $\begin{array}{l}\text { - Schmerzen im rechten Bein bei einer Gehstrecke }<200 \mathrm{~m} \\
\text { - Besserung bei Abbruch der Belastung } \\
\text { - Hin und wieder aber auch in Ruhe und nachts } \\
\text { - Kalte Füße, ausgeprägter Nagelpilz, keine Wunden } \\
\text { - Raucher (ca. } 45 \text { Packyears) }\end{array}$ \\
\hline Untersuchung: & $\begin{array}{l}\text { - rechtes Bein blass bis livide und kühler im Vergleich } \\
\text { - Fußpulse beidseits schwach ausgeprägt, rechts kaum tastbar. } \\
\text { - } \mathrm{ABI} \text { (links) }=0,8 \downarrow \quad \mathrm{ABI} \text { (rechts)=0,5 } \downarrow \text { (Normwert }=0,9)\end{array}$ \\
\hline
\end{tabular}

Hinweis: Die oben genannte Person ist frei erfunden. Etwaige Ähnlichkeiten mit realen Personen wären rein zufällig und sind nicht beabsichtigt. 


\section{Anhang 6 Custom Glass App-Screenshot: Aufgabe 2 (Angiografie)}

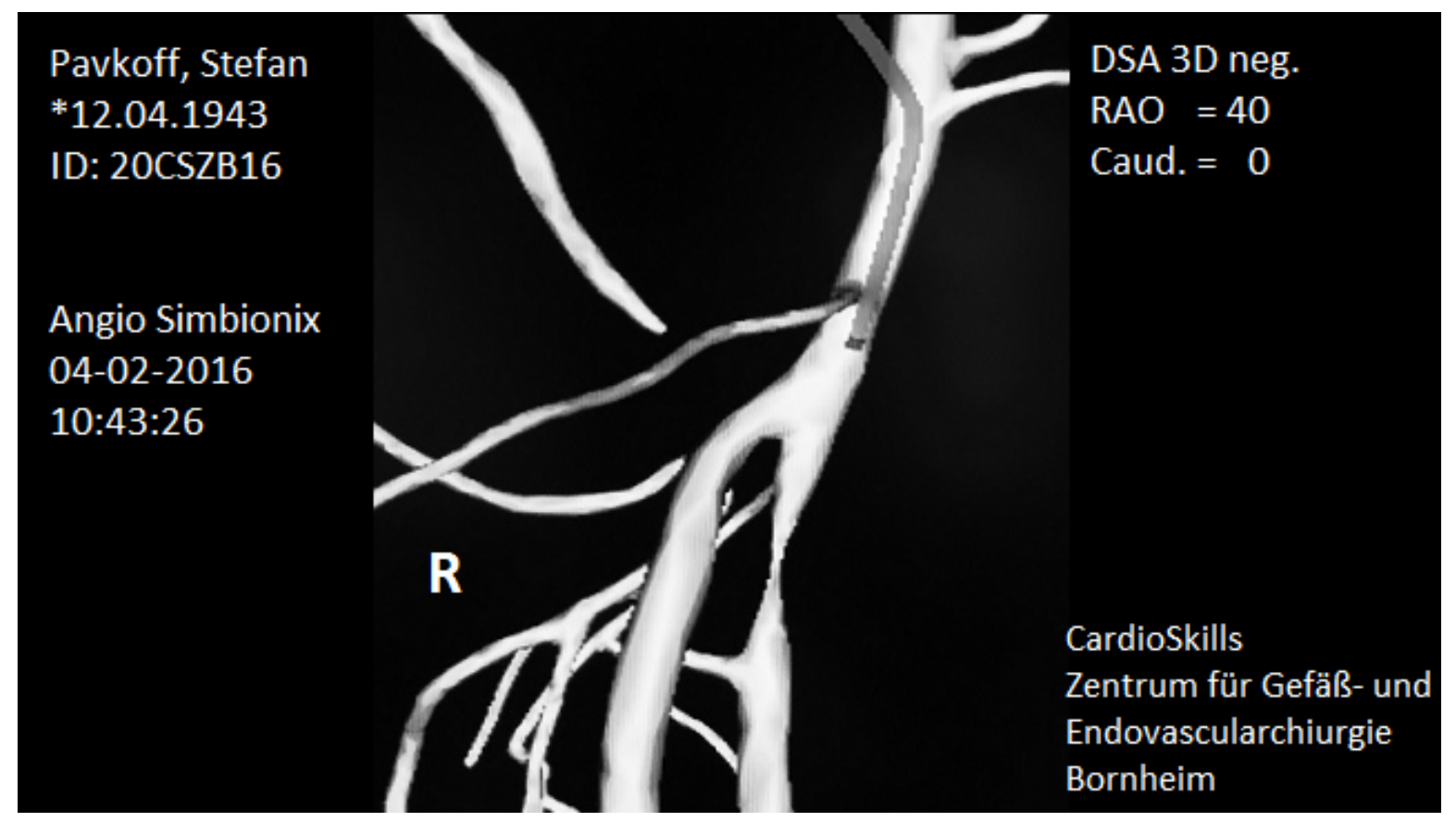

Hinweis: Die oben genannte Person ist frei erfunden.

Etwaige Ähnlichkeiten mit realen Personen wären rein zufällig und sind nicht beabsichtigt. 
Anhang 7 Custom Glass App-Screenshot: Aufgabe 3 (Laborwerte)

\begin{tabular}{|l|l|l|l|}
\hline \multicolumn{1}{|c|}{ Parameter } & \multicolumn{1}{c|}{ Wert } & \multicolumn{1}{c|}{ Einheit } & Referenzbereich \\
\hline Hämoglobin & 13,6 & $\mathrm{~g} / \mathrm{dl}$ & $13,5-17,5$ \\
Hämatokrit & 39,5 & $\%$ & $36-48$ \\
Erythrozyten & 4,64 & $\mathrm{Mill} / \mu 1$ & $4,1-5,1$ \\
Leukozyten & 5,9 & $\mathrm{Tsd} / \mathrm{\mu l}$ & $3,5-9,8$ \\
Thrombozyten & 326 & $\mathrm{Tsd} / \mathrm{\mu l}$ & $140-360$ \\
\hline Cholesterin & $\mathbf{2 2 4} \uparrow$ & $\mathrm{mg} / \mathrm{dl}$ & $\leq 200$ \\
Triglyceride & 196 & $\mathrm{mg} / \mathrm{dl}$ & $\leq 200$ \\
LDL & $\mathbf{1 7 8} \uparrow$ & $\mathrm{mg} / \mathrm{dl}$ & $\leq 160$ \\
HDL & 46 & $\mathrm{mg} / \mathrm{dl}$ & $\geq 40$ \\
\hline Kreatinin & 0,9 & $\mathrm{mg} / \mathrm{dl}$ & $\leq 1,3$ \\
Harnsäure & 5,7 & $\mathrm{mg} / \mathrm{dl}$ & $3,6-8,2$ \\
Glucose $($ Serum) & 87 & $\mathrm{mg} / \mathrm{dl}$ & $60-110$ \\
HbA1c & 6,0 & $\%$ & $4,1-6,2$ \\
\hline fT3 & $\mathbf{3 , 0 5} \uparrow$ & $\mathrm{pg} / \mathrm{ml}$ & $2,00-4,20$ \\
fT4 & $\mathbf{1 , 2 6 \uparrow}$ & $\mathrm{ng} / \mathrm{dl}$ & $0,80-1,70$ \\
TSH basal & $\mathbf{2 , 2 2} \downarrow$ & $\mu \mathrm{U} / \mathrm{ml}$ & $0,35-4,50$ \\
\hline
\end{tabular}




\section{Anhang 8 Custom Glass App-Screenshot: Aufgabe 4 (Vitalparameter)}

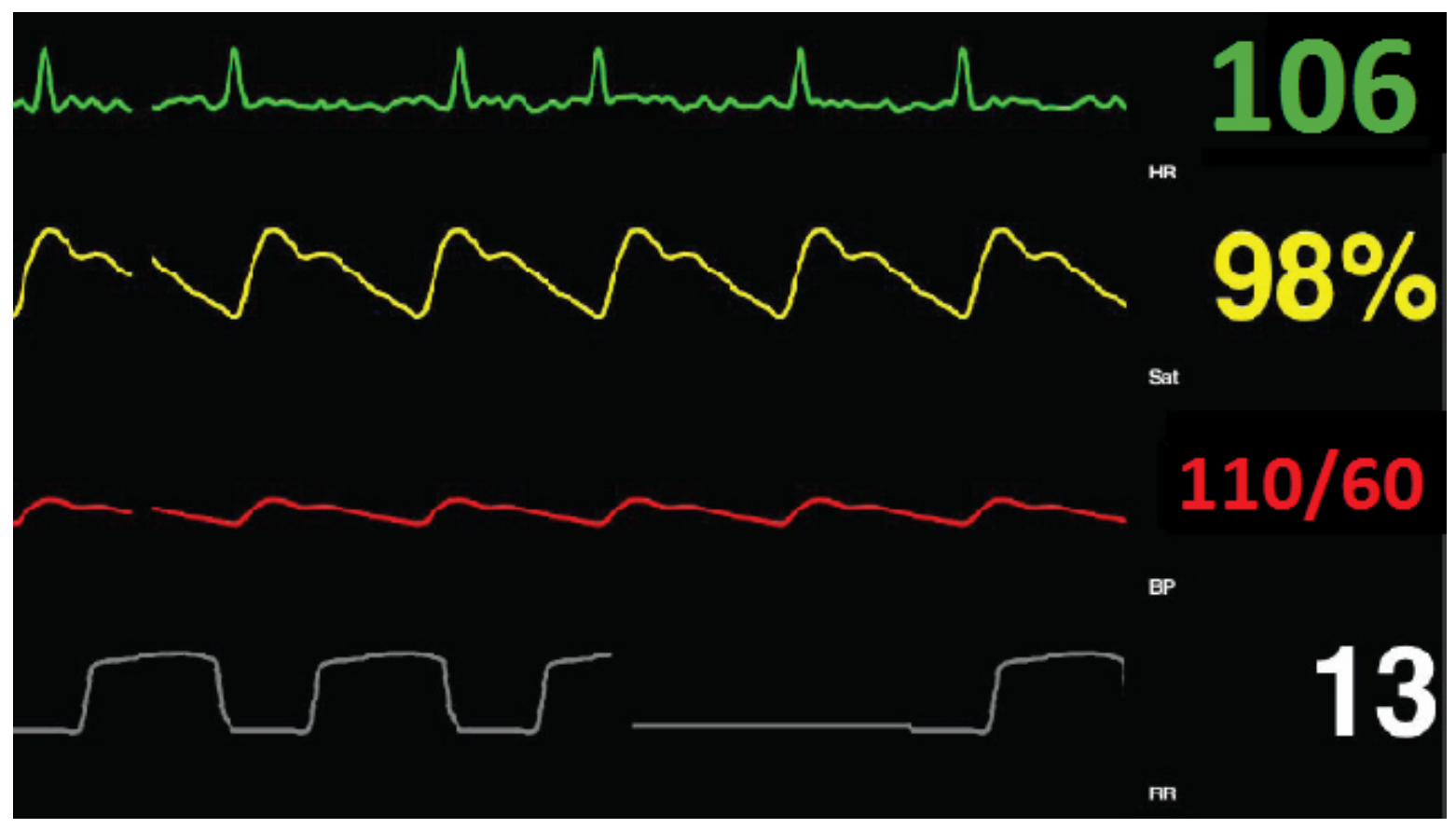




\section{Anhang 9 Custom Glass App-Screenshot: Aufgabe 5 (Fluoroskopie)}

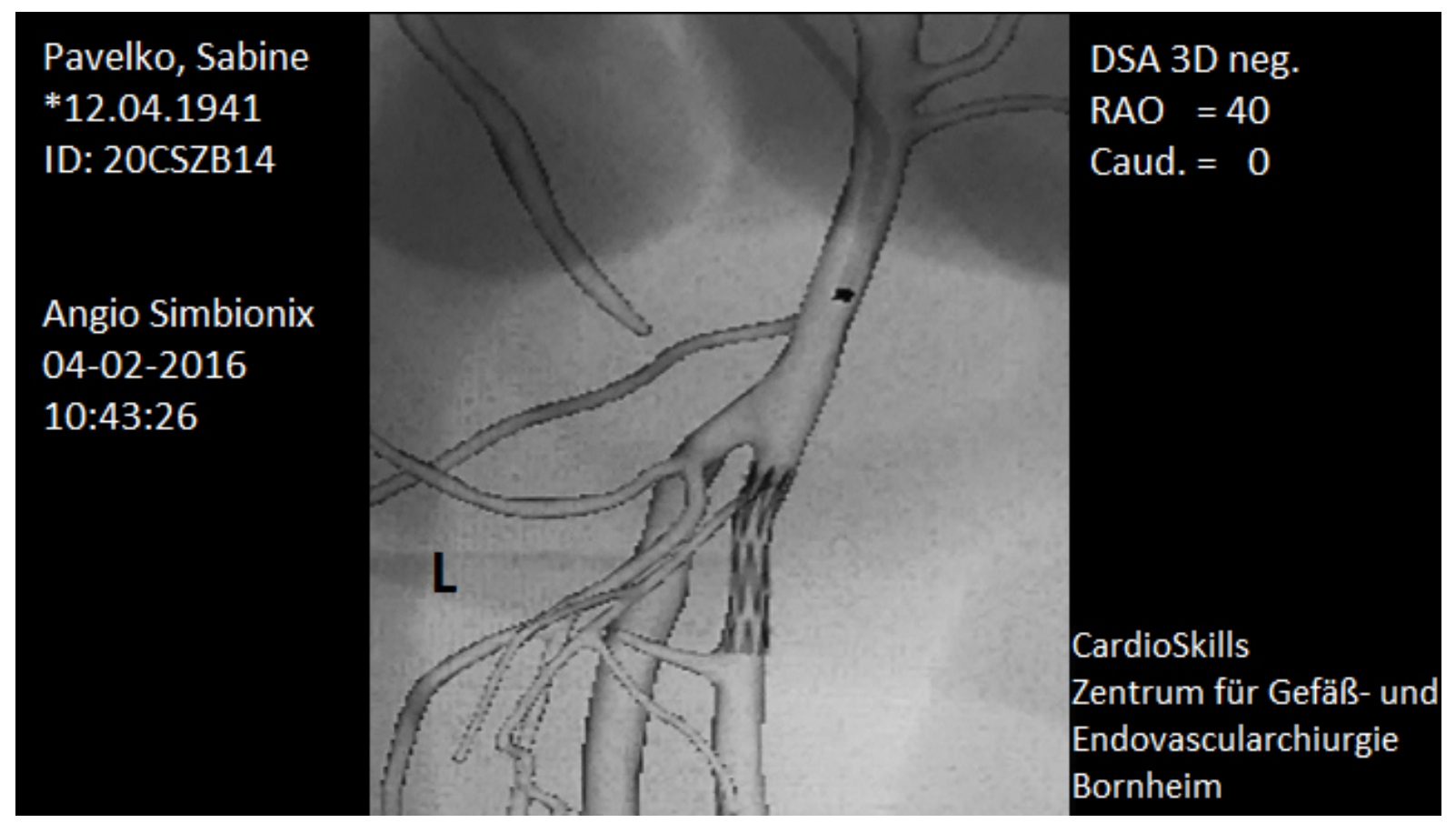

Hinweis: Die oben genannte Person ist frei erfunden.

Etwaige Ähnlichkeiten mit realen Personen wären rein zufällig und sind nicht beabsichtigt. 
Anhang 10 Custom Glass App-Screenshot: Aufgabe 6 (Foto)

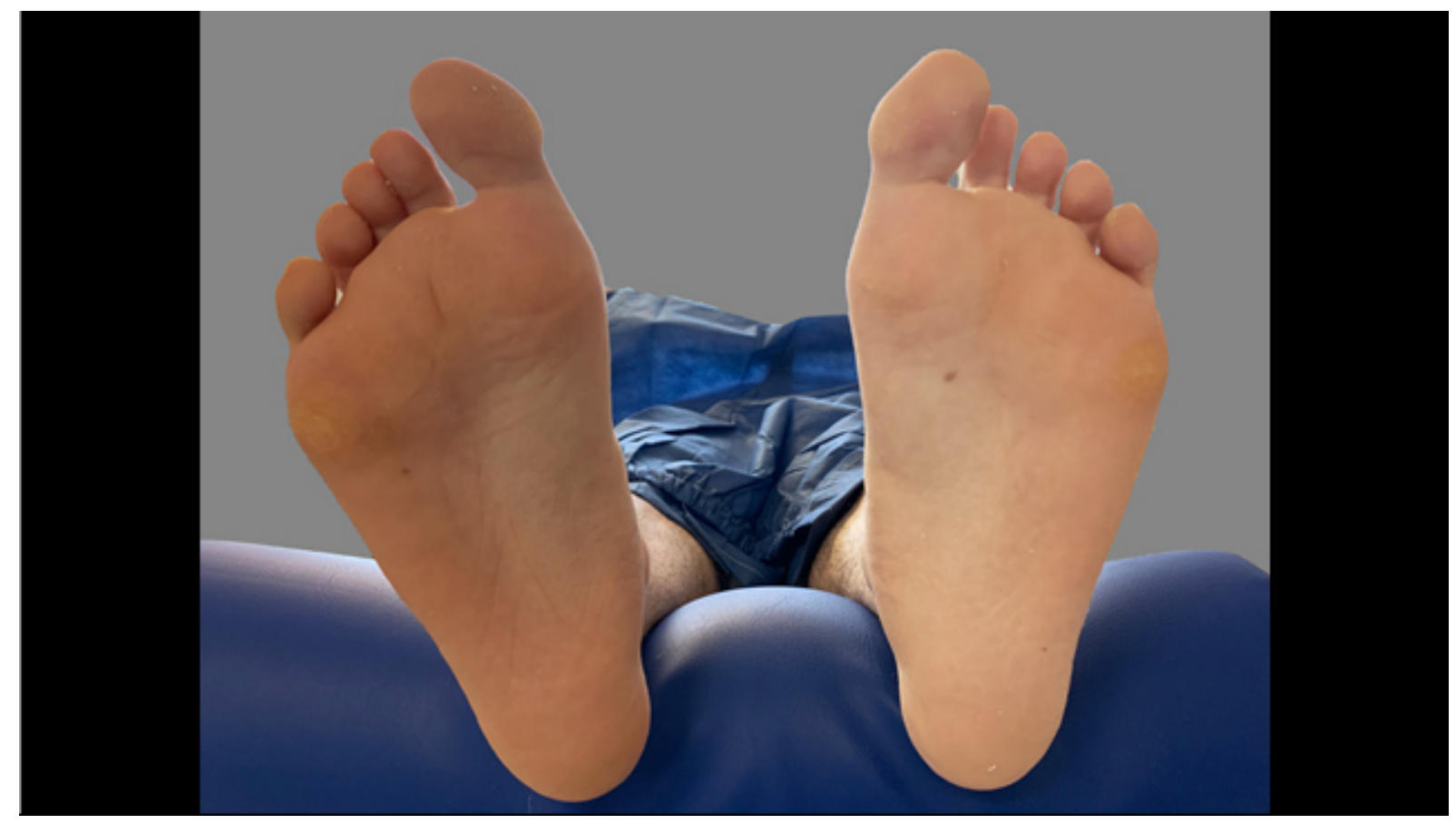




\section{LEBENSLAUF}

Name

Geburtsdatum

Geburtsort

Familienstand

$1997-2001$

$2001-2010$

$-18.06 .2010$

$2010-2011$

$2011-2017$

$-19.09 .2013$

$-13.10 .2016$

$2016-2017$

$-16.11 .2017$

$2017-2019$

seit 01.01.2020
Dennis Kaczmarek

01.05.1991

Langen (Hessen)

ledig

Grundschule in Langen

Weiterführende Gesamtschule

und gymnasiale Oberstufe

in Dreieich (Sprendlingen)

Allgemeine Hochschulreife

Zivildienst in Langen

Studium der Humanmedizin an der Goethe-Universität in Frankfurt am Main Erster Abschnitt der ärztlichen Prüfung Zweiter Abschnitt der ärztlichen Prüfung

Praktisches Jahr im Klinikum Darmstadt in den Kliniken für Anästhesiologie und Intensivmedizin, Orthopädie, Unfall- und Handchirurgie, Nephrologie und Kardiologie Dritter Abschnitt der ärztlichen Prüfung

Assistenzarzt in der Klinik für Orthopädie, Unfall- und Handchirurgie des Klinikums Darmstadt Assistenzarzt der Fachabteilung Orthopädie und Unfallchirurgie der Schön Klinik Lorsch

Frankfurt, November 2020 


\section{DANKSAGUNG}

Ebenso wie die Entwicklung neuer Technologien ein langwieriger Prozess ist, an deren Verwirklichung meist viele Köpfe beteiligt sind, so war auch die Fertigstellung dieser Dissertation ein langer Prozess, an welchem einige Personen mitgewirkt haben, denen ich an dieser Stelle danken möchte.

Mein Dank gilt zunächst Herrn Prof. Dr. Thomas Schmitz-Rixen, meinem Doktorvater, für die Annahme dieser Studie als Dissertation an der Klinik für Gefäß- und Endovascularchirurgie der Universitätsklinik Frankfurt am Main sowie für die kontinuierliche wissenschaftliche und fachliche Begleitung als erster Betreuer.

Besonderen Dank möchte ich Herrn Dr. Erhard Kaiser, meinem zweiten Betreuer, für das mir entgegengebrachte Vertrauen bei der Überlassung des Themas und für die Zurverfügungstellung von Google Glass ${ }^{\circledR}$, aussprechen. Durch seine ständige Hilfsbereitschaft, bestärkenden Worte und wertvollen Anregungen - unter anderem bei der mehrfachen, mühevollen Arbeit des Korrekturlesens - hat er maßgeblich zum Gelingen dieser Arbeit beigetragen.

Im selben Zug bedanke ich mich beim gesamten Team der CardioSkills ${ }^{\circledR}$ $\mathrm{GmbH} \&$ Co.KG für die stets freundschaftliche Zusammenarbeit und den flexiblen Zugriff auf die Simulatoren, beziehungsweise die Nutzung des Simulationszentrums zur Datenerhebung.

Auch möchte ich den 40 ehemaligen Kommilitonen danken, die sich - teilweise trotz stressiger Prüfungsvorbereitungen - die Zeit genommen haben, als Probanden an der Studie teilzunehmen und die mit ihrer Begeisterung, Neugier, aber auch konstruktiven Kritik, die Quintessenz dieser Arbeit ausmachen. Besonders Hervorheben möchte ich an dieser Stelle Benedikt Wink, der sich darüber hinaus freundlicherweise als Fotomodel zur Verfügung stellte. 
Des Weiteren gilt mein Dank Herrn Dipl. Ing. Bernd Ruprechter, Gründer des Softwareunternehmens Anvartec, für die Programmierung und den technischen Support bei der Installation der Custom Glass App.

Frau Prof. Dr. Eva Hermann, Direktorin des Instituts für Biostatistik und mathematische Modellierung der Universitätsklinik Frankfurt am Main, danke ich für die biometrische Beratung bei der Entwicklung des Studiendesigns und die fachliche Unterstützung bei der statistischen Auswertung der Studienergebnisse.

Julia Kadur möchte ich für die schnelle und unkomplizierte Hilfe bei der grammatikalischen und stilistischen Überprüfung dieser Dissertationsschrift ebenfalls danken.

Ganz besonders herzlich möchte ich mich schließlich bei meinen Freunden und meiner Familie für das stetige Interesse am Fortschritt dieser Arbeit, die ermutigenden Zusprüche, das Verständnis und die Geduld bedanken. Ohne eure vielseitige Unterstützung, schon seit Beginn des Medizinstudiums und mittlerweile über das Berufsleben als Arzt hinaus, wäre diese Arbeit nicht möglich gewesen. 


\section{Schriftliche Erklärung}

Ich erkläre ehrenwörtlich, dass ich die dem Fachbereich Medizin der Johann Wolfgang Goethe-Universität Frankfurt am Main zur Promotionsprüfung eingereichte Dissertation mit dem Titel

„Kann Google Glass $₫$ den Arbeitsablauf endovaskulärer Interventionen verbessern? Eine Pilotstudie mit Google Glass $®$ im Rahmen einer Virtual Reality Simulation."

in der Klinik für Gefäß- und Endovascularchirurgie unter Betreuung und Anleitung von Prof. Dr. Thomas Schmitz-Rixen mit Unterstützung durch Dr. Erhard Kaiser ohne sonstige Hilfe selbst durchgeführt und bei der Abfassung der Arbeit keine anderen als die in der Dissertation angeführten Hilfsmittel benutzt habe. Darüber hinaus versichere ich, nicht die Hilfe einer kommerziellen Promotionsvermittlung in Anspruch genommen zu haben.

Ich habe bisher an keiner in- oder ausländischen Universität ein Gesuch um Zulassung zur Promotion eingereicht. Die vorliegende Arbeit wurde bisher nicht als Dissertation eingereicht.

Vorliegende Ergebnisse der Arbeit wurden bisher nicht veröffentlicht.
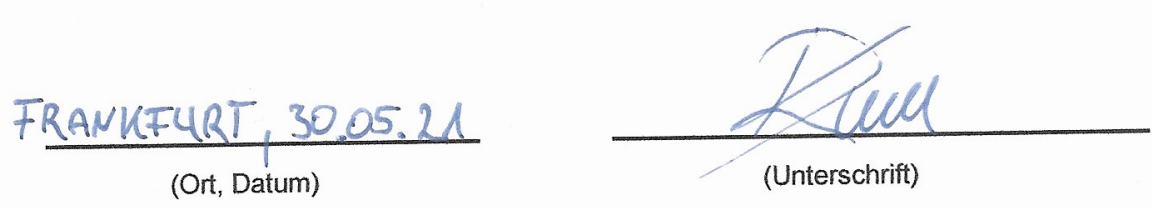